\title{
STAT3-BDNF-TrkB signalling promotes alveolar epithelial regeneration after lung injury
}

\author{
Andrew J. Paris ${ }^{1}$, Katharina E. Hayer ${ }^{2}{ }^{2}$, Joseph H. Oved ${ }^{3}$, Daphne C. Avgousti ${ }^{4}$, Sushila A. Toulmin ${ }^{5}$, \\ Jarod A. Zepp ${ }^{1,6}$, William J. Zacharias7, Jeremy B. Katzen ${ }^{1,6}$, Maria C. Basili,6, Madison M. Kremp ${ }^{6}$, \\ April R. Slamowitz ${ }^{8}$, Sowmya Jayachandran ${ }^{9}$, Aravind Sivakumar ${ }^{\circ}{ }^{9}$, Ning Dai ${ }^{10}$, Ping Wang ${ }^{1}$, \\ David B. Frank ${ }^{6,9}$, Laurence C. Eisenlohr ${ }^{5,11}$, Edward Cantu III'12, Michael F. Beers ${ }^{10}{ }^{1,6}$, \\ Matthew D. Weitzman $\mathbb{1}^{5,11}$, Edward E. Morrisey ${ }^{1,6,13,14,15}$ and G. Scott Worthen ${ }^{6,10} \bowtie$
}

\begin{abstract}
Alveolar epithelial regeneration is essential for recovery from devastating lung diseases. This process occurs when type II alveolar pneumocytes (AT2 cells) proliferate and transdifferentiate into type I alveolar pneumocytes (AT1 cells). We used genome-wide analysis of chromatin accessibility and gene expression following acute lung injury to elucidate repair mechanisms. AT2 chromatin accessibility changed substantially following injury to reveal STAT3 binding motifs adjacent to genes that regulate essential regenerative pathways. Single-cell transcriptome analysis identified brain-derived neurotrophic factor (Bdnf) as a STAT3 target gene with newly accessible chromatin in a unique population of regenerating AT2 cells. Furthermore, the BDNF receptor tropomyosin receptor kinase B (TrkB) was enriched on mesenchymal alveolar niche cells (MANCs). Loss or blockade of AT2-specific Stat3, Bdnf or mesenchyme-specific TrkB compromised repair and reduced Fgf7 expression by niche cells. A TrkB agonist improved outcomes in vivo following lung injury. These data highlight the biological and therapeutic importance of the STAT3-BDNF-TrkB axis in orchestrating alveolar epithelial regeneration.
\end{abstract}

ung function depends on the integrity of the distal alveolar epithelium $^{1,2}$. Damage to the alveolar epithelium, as occurs in pneumonia ${ }^{3}$, influenza ${ }^{4,5}$ and acute respiratory distress syndrome $e^{6,7}$, results in failure of gas exchange, which may be fatal ${ }^{8}$. A subset of patients nonetheless survive these diseases, demonstrating that clinically meaningful lung regeneration is possible ${ }^{9-11}$. The current severe acute respiratory syndrome coronavirus 2 pandemic resulting in COVID-19 has demonstrated these principles on a worldwide scale ${ }^{12,13}$. This has led to an intense focus on understanding the mechanisms of alveolar epithelial regeneration that could accelerate this process $^{14,15}$.

Multiple cell types have been identified that function as progenitor cells to regenerate alveolar epithelium following acute lung injury ${ }^{14,15}$. These include type II alveolar pneumocytes (AT2 cells) ${ }^{16-19}$, type I alveolar pneumocytes (AT1 cells) $)^{20,21}$, bronchoalveolar stem cells ${ }^{22-24}$, airway stem cells ${ }^{25,26}$ and lineage-negative progenitors ${ }^{27}$. This redundancy highlights the evolutionary importance of alveolar epithelial regeneration. Although the relative contribution of each pool of progenitors to alveolar epithelial regeneration remains to be determined, AT2 cells are indispensable for alveolar epithelial regeneration following sterile lung injury ${ }^{28}$. Furthermore, a subset of Axin2 ${ }^{+}$AT2 cells called alveolar epithelial progenitor cells (AEPs), marked by Tm4sf1 expression and fibroblast growth factor 7 (FGF7) responsiveness, were shown to contribute to alveolar re-epithelialization following sterile and infectious lung injuries ${ }^{19}$. Efforts to elucidate the mechanisms of AT2 stem cell functions will undoubtedly enhance our understanding of alveolar epithelial regeneration. For example, reports of keratin 5-positive $\left(\mathrm{Krt}^{+}\right)$ cell-mediated regeneration in areas where AT2-mediated regeneration fails ${ }^{27,29}$ suggest redundancy, even though $\mathrm{Krt5}^{+}$cell-mediated regeneration may be less efficient at restoration of normal alveolar structure and function ${ }^{27,30}$.

Under homeostatic conditions, the AT2 cell is a quiescent surfactant-producing cell ${ }^{11,32}$. Following acute lung injury, the AT2 cell functions as a progenitor cell that is capable of self-renewal and transdifferentiation into AT1 cells ${ }^{15,17,19}$, which are flat pneumocytes that facilitate gas exchange between the alveoli and adjacent capillaries $^{33}$. Despite extensive research defining the stem cell functions of AT2 cells ${ }^{17-19}$, an understanding of how changes in chromatin accessibility, transcription factor activation and gene expression in

'Division of Pulmonary, Allergy and Critical Care Medicine, Department of Medicine, Perelman School of Medicine, University of Pennsylvania, Philadelphia, PA, USA. 'Department of Biomedical and Health Informatics, Children's Hospital of Philadelphia, Philadelphia, PA, USA. ${ }^{3}$ Division of Hematology, Department of Pediatrics, Perelman School of Medicine, University of Pennsylvania, Philadelphia, PA, USA. "Division of Human Biology, Fred Hutchinson Cancer Research Center, Seattle, WA, USA. ${ }^{5}$ Department of Pathology and Laboratory Medicine, Perelman School of Medicine, University of Pennsylvania, Philadelphia, PA, USA. ${ }^{6}$ Penn-CHOP Lung Biology Institute, Perelman School of Medicine, University of Pennsylvania, Philadelphia, PA, USA. 'Division of Pulmonary Biology, Perinatal Institute, Department of Pediatrics, University of Cincinnati College of Medicine, Cincinnati, OH, USA. ${ }^{8}$ Department of Pediatrics, Children's National, Washington, DC, USA. ${ }^{9}$ Division of Cardiology, Department of Pediatrics, Perelman School of Medicine, University of Pennsylvania, Philadelphia, PA, USA. ${ }^{10}$ Division of Neonatology, Department of Pediatrics, Perelman School of Medicine, University of Pennsylvania, Philadelphia, PA, USA. "Division of Protective Immunity, Department of Pathology and Laboratory Medicine, Children's Hospital of Philadelphia, Philadelphia, PA, USA. '2Division of Cardiovascular Surgery, Department of Surgery, Perelman School of Medicine, University of Pennsylvania, Philadelphia, PA, USA. ${ }^{13}$ Penn Cardiovascular Institute, Perelman School of Medicine, University of Pennsylvania, Philadelphia, PA, USA. ${ }^{14}$ Penn Institute for Regenerative Medicine, Perelman School of Medicine, Philadelphia, PA, USA. ${ }^{15}$ Department of Cell and Developmental Biology, Perelman School of Medicine, University of Pennsylvania, Philadelphia, PA, USA.凶e-mail: worthen@email.chop.edu 
AT2 cells influence interactions with adjacent mesenchymal niche cells is needed to improve our knowledge of how the alveolar epithelium regenerates. An integrated understanding of these biological processes is essential for developing therapeutics for devastating human lung diseases that injure alveolar epithelium ${ }^{15}$.

Discerning which factors and transcriptional targets are critical to regenerating epithelial cell functions ${ }^{34,35}$ requires genome-wide analyses $^{36}$. To investigate this, we assayed the chromatin architecture of AT2 cells for changes in accessibility following acid-induced lung injury using the assay for transposase accessible chromatin and next-generation sequencing (ATAC-seq) $)^{36}$. Studies of chromatin were coupled with single-cell transcriptomic analysis of AT2 cells and mesenchymal cells to improve our understanding of the cell signalling interactions between discrete populations of regenerating AT2 and niche cells. These genome-wide approaches converged on a single pathway in which the transcription factor signal transducer and activator of transcription 3 (STAT3) increases the expression of growth factor brain-derived neurotrophic factor (BDNF) in transdifferentiating AT2 cells. Using human and murine alveolar organoids ${ }^{37}$, we demonstrated that this is a conserved pathway in epithelial regeneration. Furthermore, drug treatments aimed at BDNF-tropomyosin receptor kinase B (TrkB) signalling enhance repair following lung injury in mice, demonstrating that targeting this pathway may provide avenues for the therapy of heretofore recalcitrant diseases ${ }^{38}$.

\section{Results}

The response to acid-induced lung injury is marked by changes in AT2 chromatin architecture. We previously described a model of alveolar lung injury and repair induced by acid aspiration in which AT2 cells proliferate within $24 \mathrm{~h}$ following injury and are the progenitors to AT1 cell reconstitution ${ }^{39}$. This unilateral injury model allows mice to survive an otherwise fatal injury, and has distinct inflammatory and regenerative phases ${ }^{39}$. We hypothesized that, in response to epithelial loss, the AT2 cell adopts new stem cell functions not present at homeostasis, and that the regenerative function is facilitated by transcriptional changes resulting from alterations in chromatin architecture. We therefore isolated AT2 cells from mice $24 \mathrm{~h}$ after acid instillation (Fig. 1a,b and Extended Data Fig. 1) and compared them with AT2 cells from uninjured mice for chromatin accessibility using the ATAC-seq assay ${ }^{36}$ (Fig. 1c). We purposefully avoided $S f t p c$ lineage-labelled mice for this experiment in order to capture cells from other progenitor types that may transiently adopt characteristics of AT2 cells following injury.

Regions of accessible chromatin were substantially different in AT2 cells from mice that underwent acid-induced lung injury compared with those from uninjured mice (Fig. 1d,e). Using an unbiased pathway analysis (Fig. 1f), we identified areas of new chromatin accessibility that are associated with genes involved in cell movement and proliferation. To determine which transcription factors may occupy sites important for expression of these genes, we located binding sites using hypergeometric optimization of motif enrichment (HOMER) analysis ${ }^{40}$ (Fig. 1g). Examination of genes in areas of accessible chromatin demonstrated enrichment for lung development and alveolar epithelium-associated transcription factors including NKX2-1 (refs. ${ }^{41,42}$ ) and FOXP1 (refs. ${ }^{43,44}$ ). Binding site motifs that appear enriched following injury include motifs for STAT3, ELK4 (ref. ${ }^{45}$ ), TBX5 and SPIB. Thus, following acute lung injury, the chromatin in AT2 cells has increased accessibility to specific transcription factor binding sites and genes involved in cell proliferation.

STAT3 is important for AT2-mediated recovery from sterile and infectious lung injuries. We focused on STAT3 since our previous work highlighted the importance of the STAT3 activators granulocyte colony stimulating factor and interleukin-6 (IL-6) in alveolar epithelial regeneration ${ }^{39,46}$. Furthermore, we detected increased IL-6 in the bronchoalveolar lavage (BAL) of mice $24 \mathrm{~h}$ after acute lung injury, temporally corresponding to the initiation of alveolar regeneration $^{39}$ (Fig. 2a and Extended Data Fig. 2). To determine whether altered accessibility and accumulation of activating cytokines ${ }^{47}$ accurately predicts the activation of STAT3, we probed tissue sections obtained from acid-injured and control lungs for the presence of activated phosphorylated STAT3 (ref. ${ }^{48}$ ). Immunofluorescence staining $24 \mathrm{~h}$ after acid-induced lung injury or $9 \mathrm{~d}$ after H1N1 influenza infection revealed activation of STAT3 in AT2 cells as well as other cell types (Fig. 2b,c and Extended Data Fig. 2). We did not observe STAT3 activation in uninjured lungs (Fig. 2b,c). This observation was corroborated by evidence of STAT3 activation in AT2 cells of human explanted lungs with diffuse alveolar damage (Fig. 2d,e). Activation of STAT3 is thus an evolutionarily conserved response to lung injury.

To determine downstream effectors of STAT3 activation, we performed an unbiased pathways analysis of genes in close proximity to newly accessible STAT3 binding sites. We identified multiple gene expression pathways related to development, epithelial proliferation and insulin receptor signalling (Fig. $2 \mathrm{f}, \mathrm{g}$ ). To determine whether STAT3 activation is functionally critical for repair in AT2 epithelial cells, we generated $S f t p c^{C r E R T 2}:$ Stat $3^{\text {LoxP/LoxP }}$ mice in which a critical element of Stat 3 could be selectively deleted in AT2 cells upon administration of tamoxifen (Extended Data Fig. 3a). Previous studies using organoids showed that IL-6 (ref. ${ }^{46}$ ) promoted organoid formation and, conversely, STAT3 inhibition decreased organoid formation. However, these reports did not delineate between the relative importance of AT2-specific STAT3 compared with mesenchymal-specific STAT3. We therefore tested the importance of AT2-specific STAT3 signalling by assessing the ability of AT2 cells lacking Stat 3 to form alveolar organoids when co-cultured with wild-type fibroblasts. We observed that AT2 cells lacking Stat 3 formed significantly fewer organoids than those that retained Stat3 (Extended Data Fig. 3b). Thus, AT2-specific STAT3 functionality is necessary for alveolar organoid formation.

Fig. 1 The response to injury is marked by significant changes in AT2 chromatin architecture. a, AT2 cells were isolated from single-cell suspensions of murine lung tissue (three uninjured mice and two injured mice) using fluorescence activated cell sorting. Nuclei were isolated and incubated with Tn5 transpose followed by amplification of fragments and next-generation sequencing (NGS). b. Staining of isolated cells for the AT2 markers pro-surfactant protein C (proSFTPC) and dendritic cell lysosomal associated membrane glycoprotein (DC-LAMP) confirmed that $>97 \%$ of isolated cells were AT2 cells (100 cells per stain were analysed in $n=3$ independent experiments). Data are shown as means \pm s.e.m. DAPI, $4^{\prime}, 6$-diamidino-2-phenylindole. c, ATAC-seq was performed on AT2 cells from uninjured mice (quiescent) and from mice $24 \mathrm{~h}$ after acid-induced lung injury (regenerative). Next-generation sequencing reads were aligned and uninjured and injured sequences were compared. $\mathbf{d}, \mathbf{e}$, Quantitation of shared and unique regions of accessible chromatin (d) and heat maps of accessibility (e). TSS, transcription start site; TTS, transcription termination site. f, Pathways enriched in injured AT2 cells. Ingenuity pathway analyses of genes associated with differentially accessible chromatin following acute lung injury demonstrated that newly accessible areas of the genome encode proteins important for alveolar epithelial regeneration. $\mathbf{g}$, HOMER analysis of accessible chromatin demonstrated common enrichment for alveolar epithelium-associated transcription factors, including NKX2.1, FOXF1 and FOXP1. After injury, STAT3, TBX5 and SPIB chromatin accessibility becomes increasingly enriched. Statistical significance was determined with the findMotifGenome.pl script in the HOMER software package, with adjustment for multiple comparisons. 
To test the hypothesis that AT2 cell-specific STAT3 promotes repair in vivo, Sftpc CreERT2 Stat $^{\text {LoxP/LoxP }}$ mice were administered tamoxifen or corn oil (vehicle control) $18 \mathrm{~d}$ before acid-induced

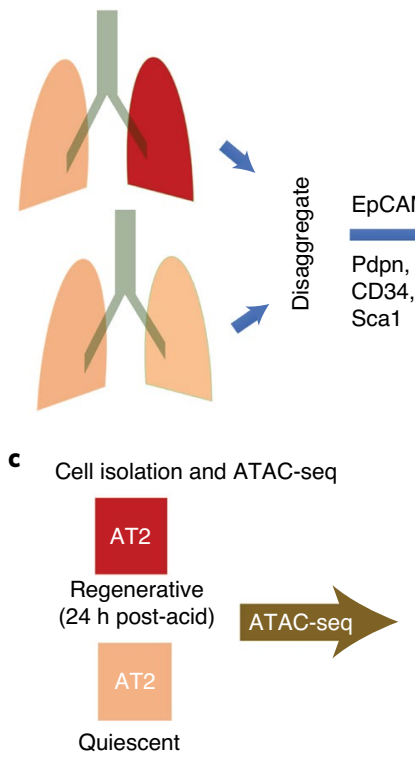

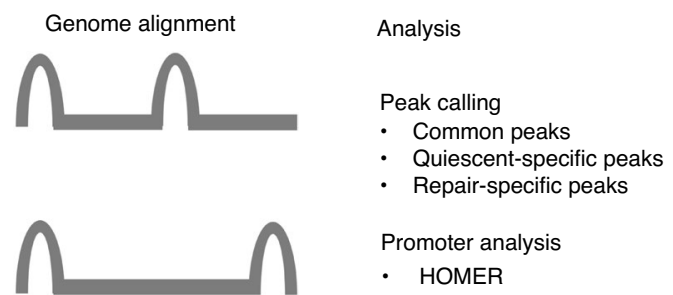

d

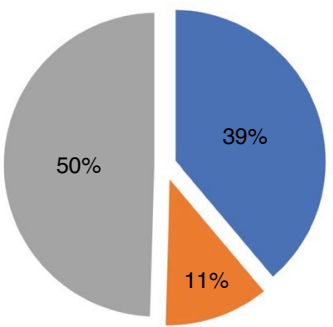

- Injured $=$ Uninjured $=$ Common
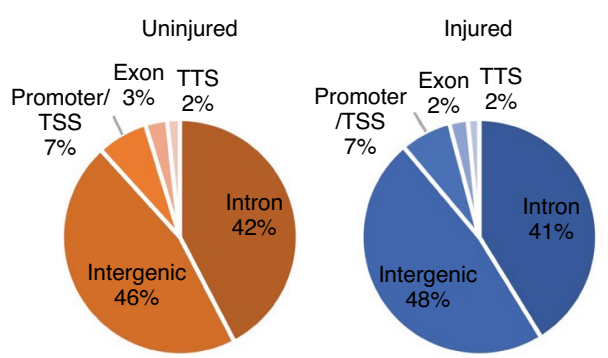

$\mathbf{g}$

\begin{tabular}{|c|c|c|c|}
\hline $\begin{array}{l}\text { Transcription } \\
\text { factor }\end{array}$ & Motif & $\begin{array}{l}P \text { value } \\
\text { (injured) }\end{array}$ & $\begin{array}{l}P \text { value } \\
\text { (uninjured) }\end{array}$ \\
\hline NKX2.1 & G & $1 \times 10^{-232}$ & $1 \times 10^{-267}$ \\
\hline FOXF1 & 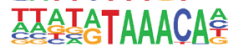 & $1 \times 10^{-110}$ & $1 \times 10^{-130}$ \\
\hline FOXP1 & 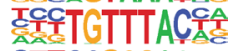 & $1 \times 10^{-79}$ & $1 \times 10^{-118}$ \\
\hline STAT3 & FITCCEGGAA & $1 \times 10^{-44}$ & $1 \times 10^{-5}$ \\
\hline ELK4 & 숭순TCCGGT & $1 \times 10^{-47}$ & $1 \times 10^{-2}$ \\
\hline TBX5 & A & $1 \times 10^{-78}$ & $1 \times 10^{-7}$ \\
\hline SPIB & AMACANA & $1 \times 10^{-32}$ & $1 \times 10^{-9}$ \\
\hline
\end{tabular}

- HOMER lung injury. Distal alveoli of unchallenged lungs appeared histologically unaltered after deletion (Extended Data Fig. 4). While control mice showed normal rapid repair (Fig. 3a, top panels), b
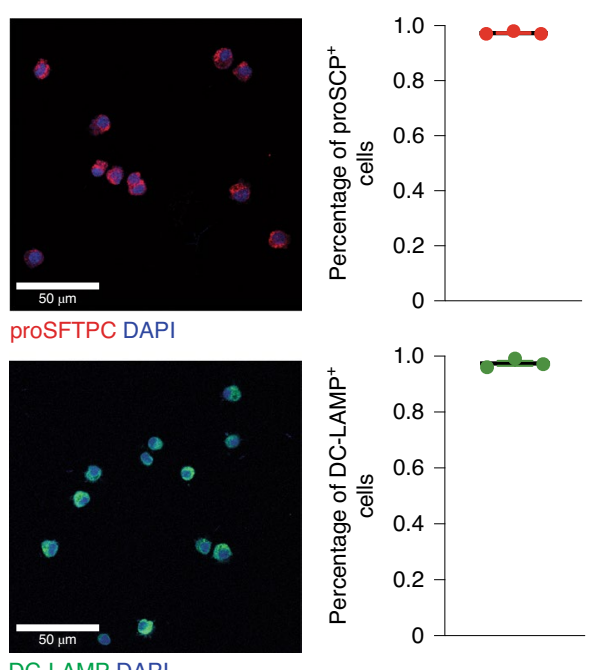

DC-LAMP DAPI e

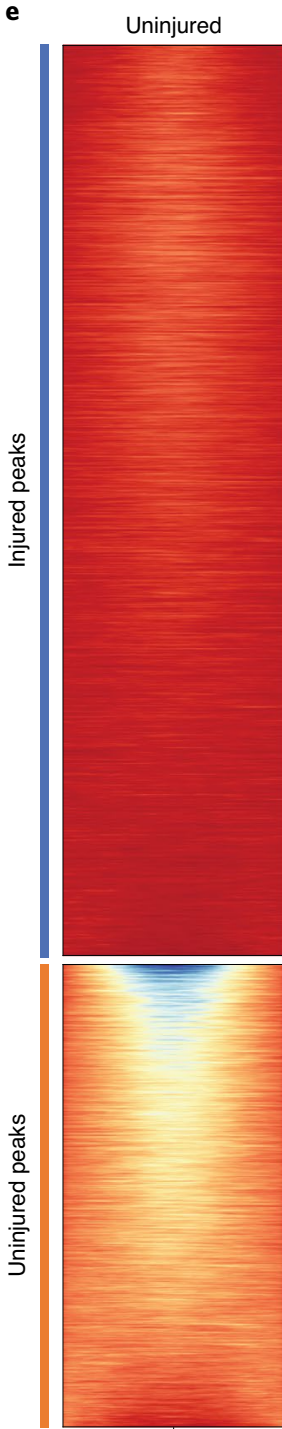

Peak centre

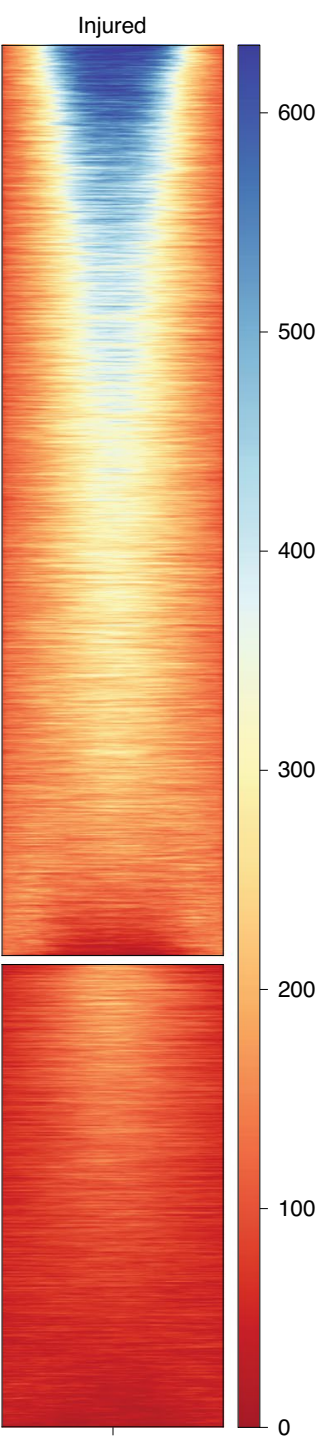

Peak centre

600

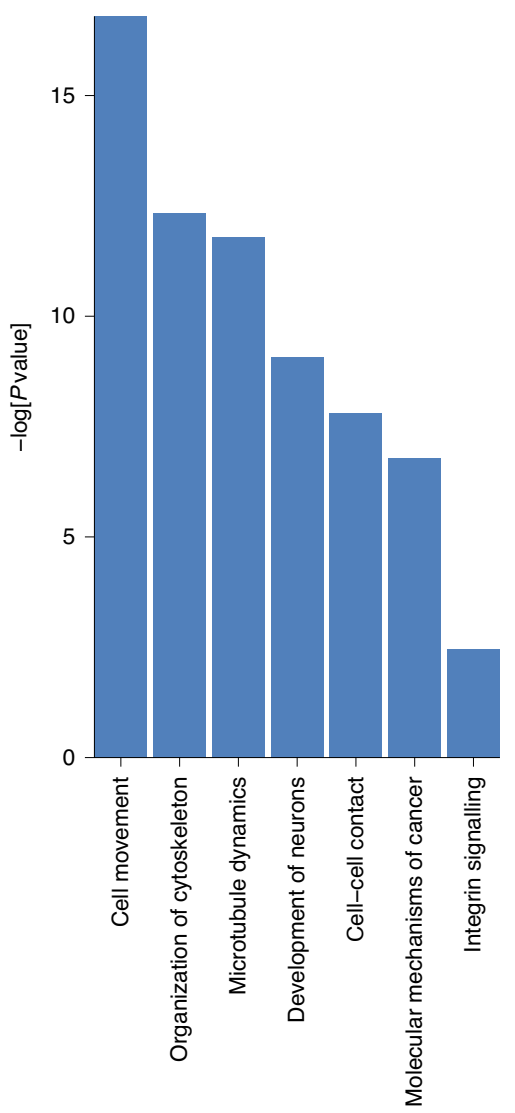




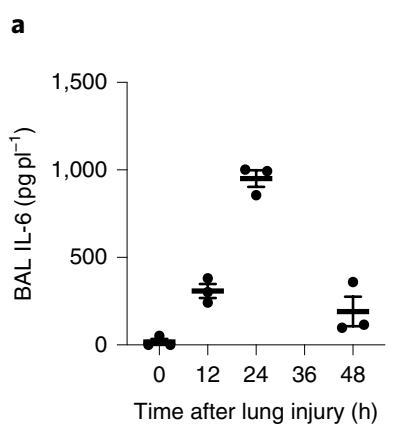

b

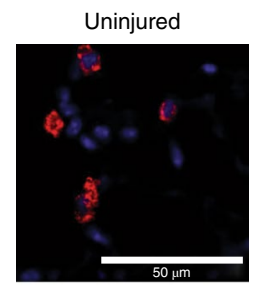

$24 \mathrm{~h}$ post-acid

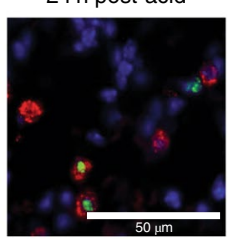

9 d post-influenza

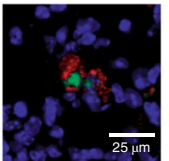

pSTAT3 proSFTPC DAP

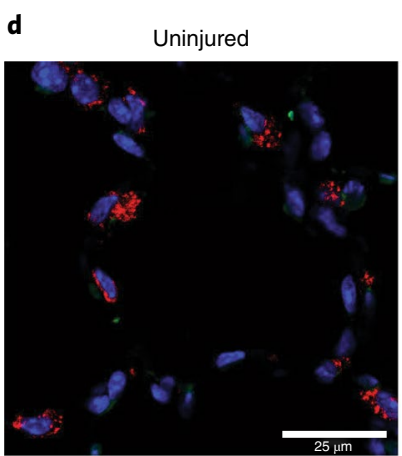

f

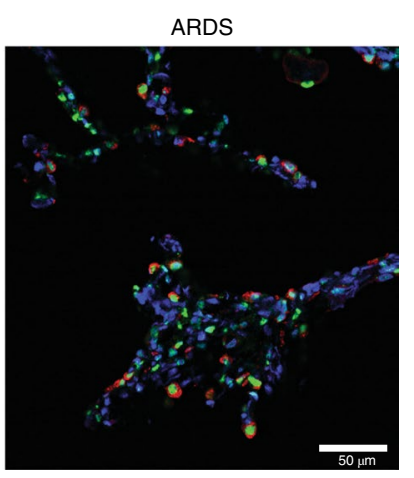

DSTAT3 ProSFTPC DAPI

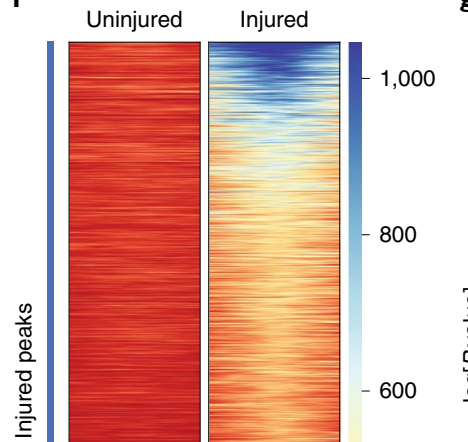

Uninjured

Injured
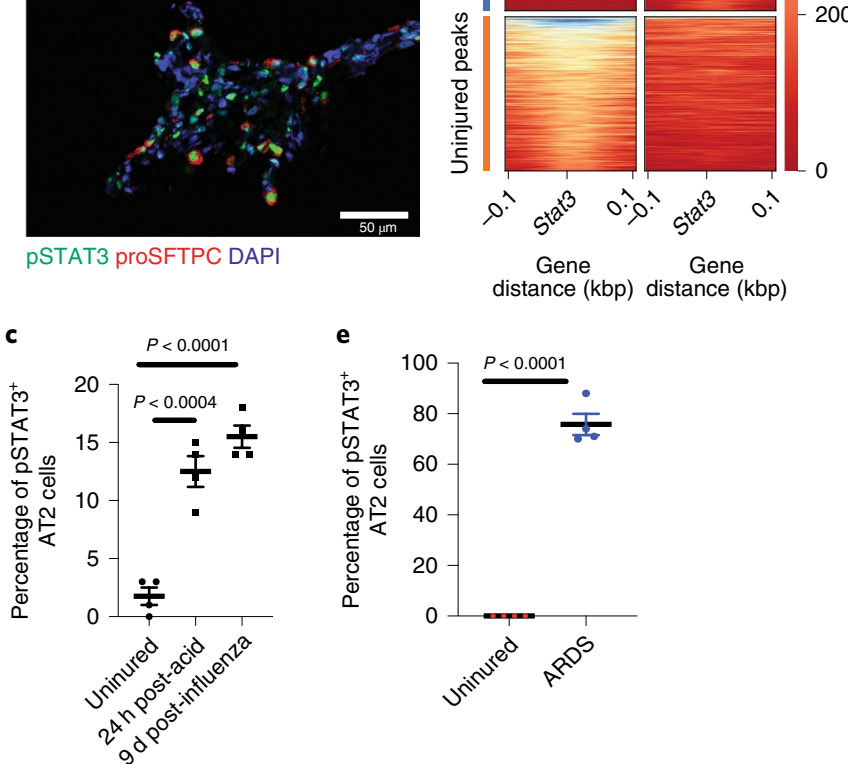

g

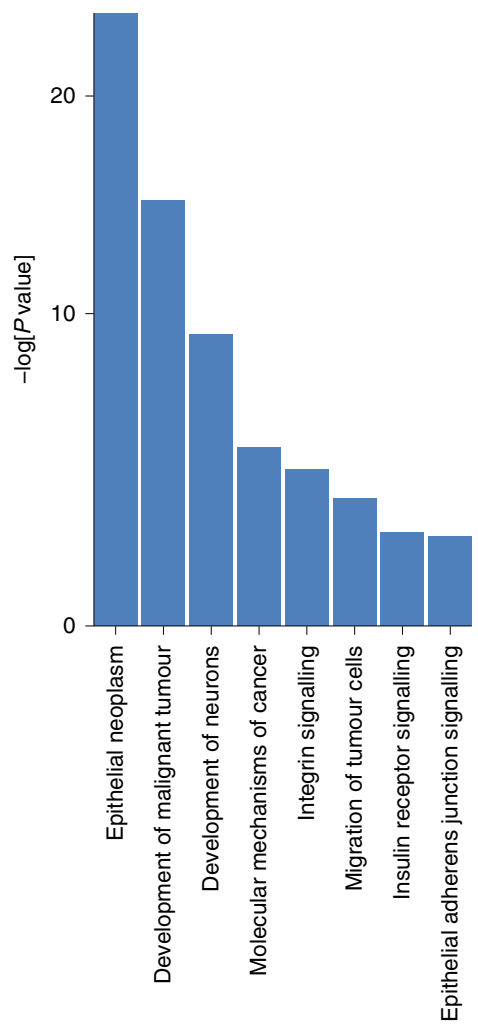

Fig. 2 | STAT3 regulates key pathways in alveolar epithelial regeneration. a, IL- 6 is undetectable in uninjured lungs. Levels of IL-6 in BAL fluid peak at $24 \mathrm{~h}$ after acid-induced lung injury ( $n=3$ mice per time point). b,c, Images (b) and quantitation (c) showing that C57BL/6 mice that underwent acid-induced lung injury had activated phosphorylated STAT3 (pSTAT3) in AT2 cells ( $P=2.9 \times 10^{-5}$ for 9 d post-infection versus uninjured). There was minimal pSTAT3 in AT2 cells of uninjured mice ( $n=4$ mice per group). d,e, Images (d) and quantitation (e) showing that human samples with diffuse alveolar damage had increased AT2-specific pSTAT3 $\left(P=1.8 \times 10^{-6}\right)$ that was not present in uninjured samples ( $n=4$ patients per group). ARDS, acute respiratory distress syndrome. $\mathbf{f}, \mathbf{g}$, Unbiased pathway analyses demonstrated newly accessible STAT3 binding sites (heat map in $\mathbf{f}$ ) adjacent to genes that control key biological mechanisms including proliferation and migration shown in $\mathbf{g}$. kbp, kilobase pairs. In a, c and e, data are shown as means \pm s.e.m. Statistical significance was determined by two-tailed Student's t-test (c and e).

Sftpc $c^{\text {CreERT2}}$ :Stat $3^{\text {LoxP/LoxP }}$ mice had severe histological changes consistent with repair failure after acid-induced lung injury (Fig. 3a, bottom panels). This was accompanied by decreased staining for AT1 cells and significantly higher lung injury scores at later time points in mice lacking Stat3 specifically in AT2 cells (Fig. 3b,c).

Since the epithelial layer represents the major permeability barrier within the alveolus ${ }^{49,50}$, we measured BAL protein as an indicator of permeability and confirmed that the epithelial permeability barrier fails to re-form in the absence of STAT3 (Fig. 3d). As alveolar epithelium regeneration is dependent on AT2 proliferation ${ }^{16,31,39}$, we quantified proliferation by Ki67 staining (Fig. 3e,f) and found significantly fewer proliferating AT2 cells in the absence of STAT3. We did not observe changes in the absolute number of AT2 cells $1 \mathrm{~d}$ after acid-induced lung injury in mice with an AT2 cell-specific
Stat3 deletion (Fig. 3g), suggesting that changes in initial AT2 survival were not altered. Furthermore, no differences in the American Thoracic Society (ATS) lung injury scores (Fig. 3c), BAL protein (Fig. 3d), BAL neutrophils (Fig. 3h) or BAL IL-1 $\beta$ (Fig. 3i) were observed $24 \mathrm{~h}$ after acid-induced lung injury, showing that loss of Stat3 in AT2 cells does not alter the acute inflammatory response.

To determine whether outcomes following chronic infectious injury would be similarly dependent on STAT3, we tested the effect of H1N1 Influenza infection on Sftpc $c^{\text {CreERT2 }}$ :Stat $3^{\text {LoxP/LoxP }}$ and $S f t p c^{C r e E R T 2}$ mice. At $18 \mathrm{~d}$ after tamoxifen pretreatment, mice were exposed to H1N1 $\left(5 \times 10^{-5}\right.$ haemagglutination units (HAU) per mouse; PR8 strain) and observed for $21 \mathrm{~d}$ post-infection. Mice with Stat3-deficient AT2 cells had significantly impaired survival beginning on day 8 after infection (Fig. 3j). Histological analysis 
of lungs obtained at $14 \mathrm{~d}$ after infection revealed extensive damage when Stat 3 was deleted from AT2 cells compared with controls (Fig. 3k). Thus, AT2-specific STAT3 activation and transcriptional activity is necessary for recovery from both sterile and infectious lung injuries.

Single-cell RNA sequencing (scRNA-seq) identifies BDNF as important for alveolar regeneration. To identify relevant STAT3 target genes expressed after acute lung injury, we performed scRNA-seq to identify transcriptomic changes in AT2 cells after acid-induced lung injury (Fig. 4a,b). A recent report suggested that a specific subset of AT2 cells that express Tm4sf1 are important in alveolar epithelial regeneration ${ }^{19}$. Under the same conditions used for isolating cells for ATAC-seq (Fig. 1a), we observed discrete subpopulations of cells, including those representing putative $\mathrm{AEPs}^{19}$ (Fig. 4c), following acid-induced lung injury. Cluster 2 (Fig. 4b), which we termed the proliferating AT2 cell population, contained proliferating AT2 cells and Ccnd1 expression was restricted to this cluster (Fig. 4b,c and Extended Data Fig. 5). Furthermore, unbiased pathways analysis of this cluster showed enrichment for genes that regulate proliferation (Extended Data Fig. 6). We focused our analysis on cluster 3 (Fig. 4b), which we termed the transdifferentiating population. This subpopulation is unique in its simultaneous expression of Tm4sf1, Sftpc and Pdpn (Fig. $4 \mathrm{a}-\mathrm{c}$ and Extended Data Fig. 5), similar to a previously reported population of transdifferentiating AT2 cells observed after lipopolysaccharide-induced lung injury ${ }^{51}$. These data effectively identified a subpopulation of AT2 cells whose transcriptomic profile is most consistent with transdifferentiating alveolar epithelium.

To understand the interdependence of chromatin changes after acute lung injury, STAT3-mediated gene expression and alveolar epithelial regeneration, we cross-referenced our ATAC-seq dataset with the transcriptomic profiles of the AT2 cells in cluster 3 (Fig. 4d). We identified $B d n f^{52}$ as being the only bona fide STAT3 target gene ${ }^{53-56}$ whose chromatin is newly accessible after lung injury (Fig. $4 \mathrm{~d}$ and Supplementary Table 1) and whose expression is restricted to the regenerating population of cells. Our ATAC-seq analysis revealed that the $B d n f$ locus becomes more accessible following injury (Fig. 4e), suggesting that increased chromatin accessibility contributes to increased expression after injury. Furthermore, AT2 cells isolated from tamoxifen-treated $S f t p c^{\text {CreERT2}}$ :Stat $3^{\text {LoxP/LoxP }}$ had a significant decrease in $B d n f$ expression compared with mice that did not receive tamoxifen (Fig. 4f). We also observed that BDNF release following acid-induced injury was significantly attenuated in mice with an AT2-specific Stat3 deletion (Fig. $4 \mathrm{~g}$ ). Bdnf is thus the only STAT3-regulated gene whose expression is restricted to a transcriptionally unique AT2 cell population that expresses $\operatorname{Tm} 4 s f 1$, as well as markers of both AT1 and AT2 cells.
The BDNF-TrkB axis promotes alveolar organoid formation. To test whether BDNF might enhance alveolar repair, we used a system to generate alveolar organoids by co-culturing AT2 cells with pulmonary mesenchymal cells ${ }^{37}$. This reductionist approach facilitates the study of epithelial-mesenchymal cross-talk in alveolar epithelial regeneration. The addition of recombinant BDNF to organoid media increased the efficiency and size of murine and human organoid formation (Fig. 4h,i).

Using $B d n f$ Cre-R26lslTdTomato mice, we confirmed that a small percentage of AT2 cells express $B d n f$ after acid-induced lung injury (Fig. 5a and Extended Data Fig. 7), as suggested by scRNA-seq and ATAC-seq (Fig. 4c,e). To characterize the impact of AT2 cell-specific $B d n f$, we generated an $S f t p c^{C r e E R T 2}: B d n f^{L O x P / L o x P}$ model. Mice lacking AT2-specific Bdnf had worse histological outcomes following acid-induced lung injury, as reflected by significantly worse ATS lung injury scores (Fig. 5b). As expected, BAL levels of BDNF were significantly reduced in the tamoxifen-exposed $S f t p c^{\text {CreERT2 }}: B d n f^{\text {LoxP/LoxP }}$ mice (Fig. 5c). Furthermore, tamoxifen-exposed Sftpc CreERT2: $B d n f^{\text {LoxP } / L o x P}$ mice maintained significantly elevated BAL protein levels (Fig. 5d). Although overall AT2 cell numbers were unchanged following acid-induced lung injury in the absence of Bdnf (Fig. 5e), there was a significant decrease in AT2 proliferation $5 \mathrm{~d}$ after acidinduced lung injury (Fig. 5f). We saw similar histological outcomes $21 \mathrm{~d}$ post-influenza infection (Fig. $5 \mathrm{~g}$ ) and increased KRT5 pods (Fig. 5h) when $B d n f$ was selectively deleted in AT2 cells.

Since BDNF increased alveolar organoid formation, we sought to determine whether TrkB, the receptor for BDNF, is expressed on AT2 and mesenchymal cells. Reanalysis of scRNA-seq data did not identify significant TrkB expression in AT2 cells (Extended Data Fig. 5). However, analysis of scRNA-seq data from mesenchymal cells in the mouse lung ${ }^{46}$ revealed that $\operatorname{Trk} B$ is expressed in a subset of cells (Fig. 6a-c) that were previously identified as mesenchymal alveolar niche cells (MANCs). These cells have been identified as essential for organoid formation and alveolar regeneration after bleomycin-induced lung injury ${ }^{46}$. Unbiased pathways analysis of TrkB-expressing mesenchymal cells showed enrichment for genes regulating respiratory function, organ morphology and embryonic development (Fig. 6d). Expression of TrkB on mesenchymal cells was confirmed using a TrkB Cre-Rosa26TdTomato mouse, which successfully identified platelet-derived growth factor receptor $\alpha$ (PDGFR $\alpha$ ) cells with increased TrkB expression (Fig. $6 \mathrm{e}, \mathrm{f})$. Using flow cytometry, we identified a significant expansion in TrkB-expressing cells in the lung $24 \mathrm{~h}$ after acid-induced lung injury (Fig. $6 \mathrm{~g}$ and Extended Data Fig. 8). Specifically, we found that $11.69 \%$ of MANCs and, more broadly, $1.25 \%$ of all non-MANC mesenchymal cells, expressed TrkB.

These data suggest that BDNF derived from transdifferentiating AT2 cells influences mesenchymal cells to enhance alveolar repair.

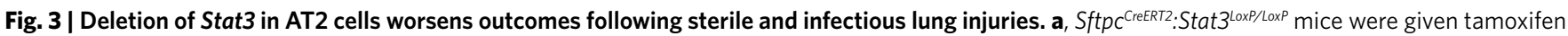
in corn oil or corn oil alone $18 \mathrm{~d}$ before acid-induced lung injury ( $n=3$ mice per group). Haematoxylin and eosin staining showed that control mice had histological injury that resolved by day 5 post-injury. Mice that received tamoxifen resulting in AT2-specific Stat3 deletion had persistent and pronounced cellular, proteinaceous and haemorrhagic infiltrates that obscured the alveolar structures. b. PDPN staining revealed that mice lacking Stat3 had decreased AT1 populations at 3 and $5 \mathrm{~d}$ following acid-induced lung injury compared with controls ( $n=3$ mice per group). c, ATS lung injury scores were increased at later time points following acid-induced lung injury in mice lacking Stat3 ( $n=3$ mice per group per time point). d, BAL protein from the mice described in a showed a non-resolving increase in alveolar protein following acid-induced lung injury in mice that lacked AT2-specific Stat3 ( $n=3$ mice per group per time point). e-g, Imaging (e) and plots of the percentage of Ki67+ AT2 cells (f) and the number of AT2 cells per $20 \times$ field ( $\mathbf{g}$ ) show that the percentage of

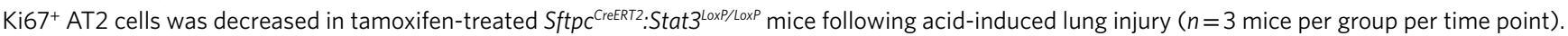
$\mathbf{h}, \mathbf{i}$, BAL neutrophils (h) and IL-1 $\beta$ levels (i) following acid-induced lung injury were not changed by the presence or absence of functional AT2-specific STAT3 ( $n=3$ mice per group per time point). $\mathbf{j}$, Sfptc ${ }^{\text {CreERT2 }}$ and Sftpc ${ }^{\text {CreERT2:Stat } 3 \text { LoxP/LoxP }}$ mice were given tamoxifen $18 \mathrm{~d}$ before intranasal infection with PR8 influenza ( $5 \times 10^{-5} \mathrm{HAU}$ per mouse). Mortality was significantly higher in the mice that lacked AT2-specific STAT3 compared with controls ( $n=10$ mice per group). k, At $21 \mathrm{~d}$ post-infection, the mouse lacking AT2-specific Stat3 (one mouse) had distorted distal alveolar lung architecture with cellular infiltrate and cystic structures that were not observed in controls (ten mice). In $\mathbf{c}, \mathbf{d}$ and $\mathbf{f}-\mathbf{i}$, data are shown as means \pm s.e.m. Statistical significance was determined by two-tailed Student's $t$-test ( $\mathbf{c}, \mathbf{f}$ and $\mathbf{g}$ ), with two-way ANOVA (d) and with the log-rank (Mantel-Cox) test (j). 
To test this hypothesis, we studied the impact of TrkB inhibition on alveolar organoid formation in vitro. Addition of ANA-12-a small molecule antagonist of $\operatorname{TrkB}^{57}$ - completely abrogated organoid formation in both murine and human alveolar organoid systems (Fig. 6h-k).
To test the importance of TrkB signalling in PDGFR $\alpha$ cells for alveolar repair, we generated a $P d g f r a^{\text {CreERT2}}: T r k B^{\text {LoxP/LoxP }}$ murine model. Mice lacking PDGFR $\alpha$ cell-specific TrkB had non-resolving pathology following our acid-induced lung injury model from which mice are usually able to recover (Extended Data Fig. 9a).

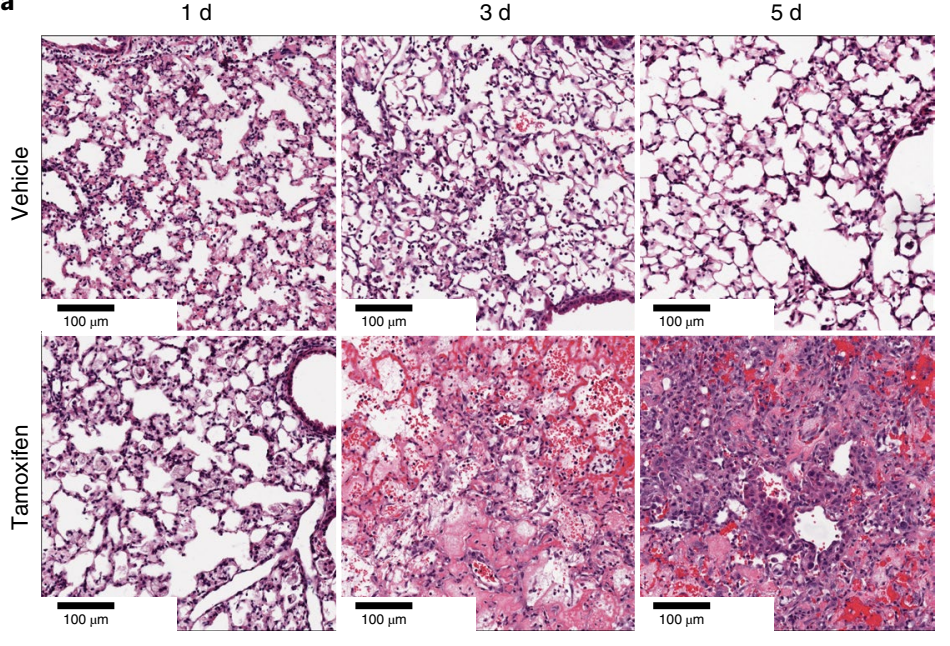

c

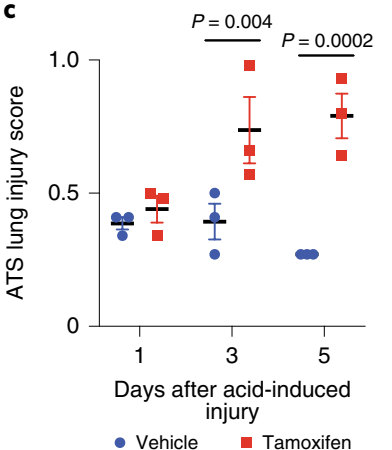

e

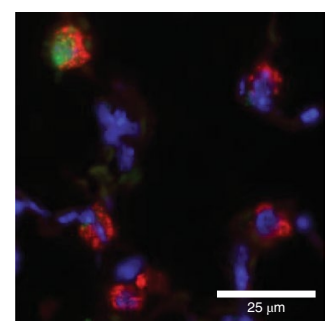

proSftpc Ki67 DAPI

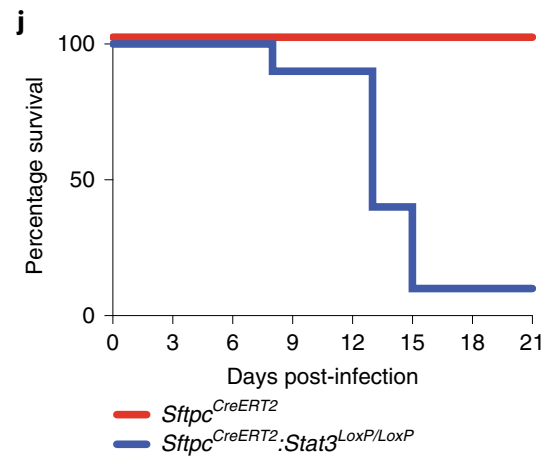

b

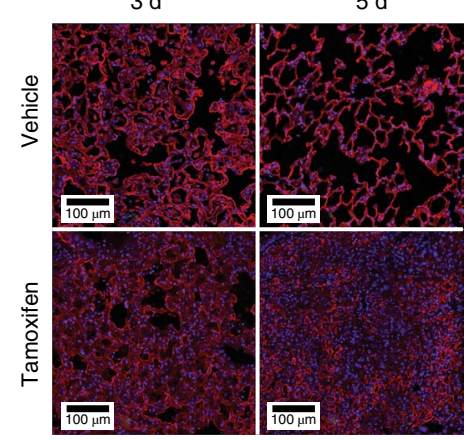

h
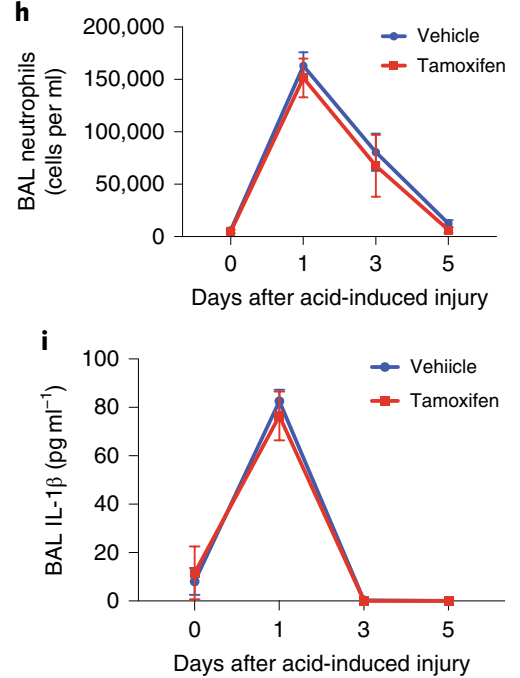
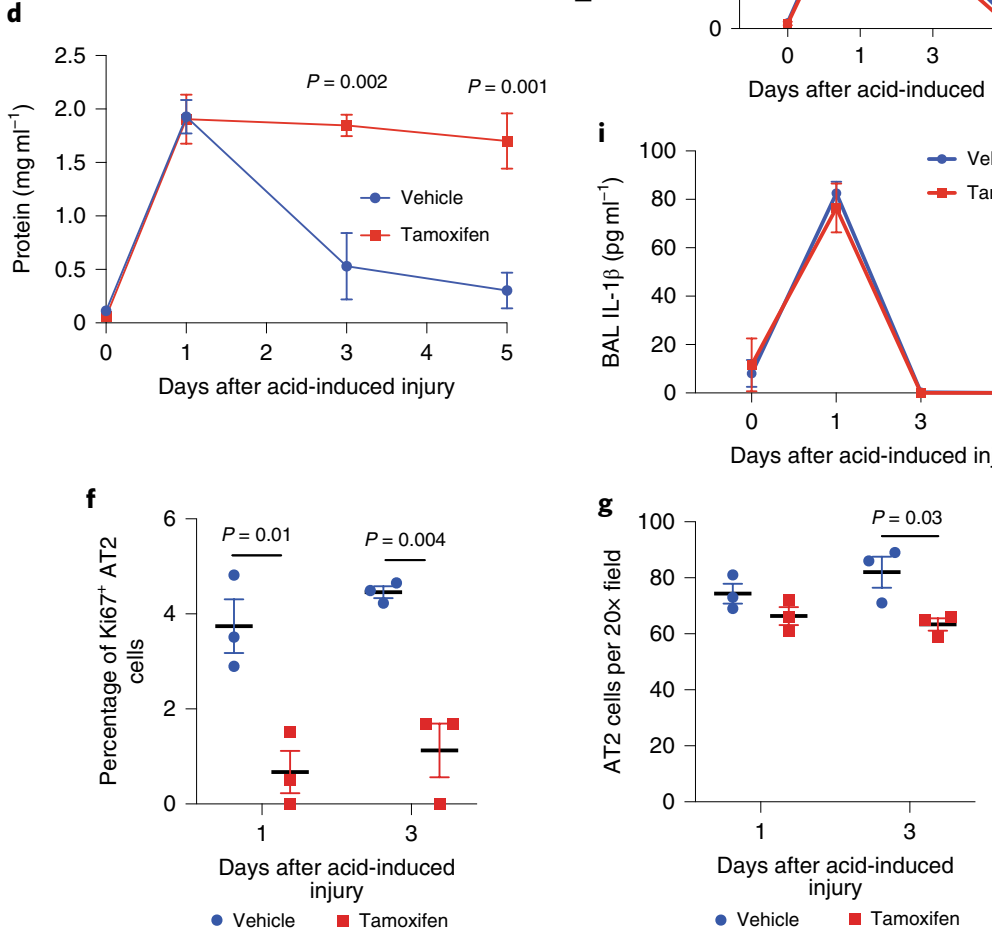

Days after acid-induced

Tamoxifen

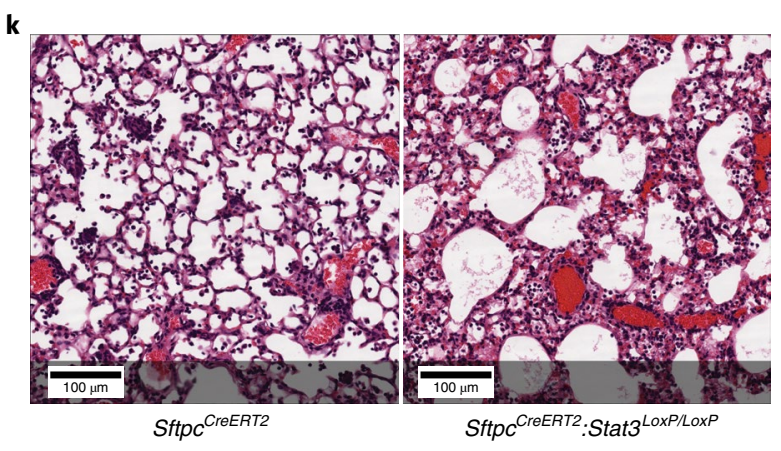


When tamoxifen-exposed $P d g f r a^{C r e E R T 2}: \operatorname{Trk} B^{\text {LoxP/LoxP }}$ mice were exposed to PR8 influenza, we observed worse histological outcomes, decreased podoplanin (PDPN) staining (Extended Data Fig. 9b,c) and increased Krt5 pods (Extended Data Fig. 9d). These data corroborate the importance of BDNF-TrkB signalling in recovery from sterile and infectious lung injuries.

Since FGF7 has been identified as an essential MANC-derived cytokine ${ }^{19,46}$ promoting alveolar epithelial regeneration, we examined the impact of BDNF on FGF7 expression. Addition of recombinant BDNF to isolated mesenchymal cells from the organoid model shown in Fig. 4h significantly increased the expression of Fgf7 (Fig. 7a), providing a mechanistic basis for the observation that BDNF promotes alveolar epithelial regeneration. Similarly, PDGFR $\alpha$ cells from tamoxifen-exposed $S f t p c^{\text {CreERT2 }}$ :Stat $3^{\text {LoxP/LoxP }}$,

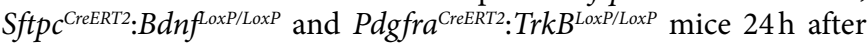
exposure to acid-induced lung injury had reduced expression of Fgf7 in PDGFR $\alpha^{+}$cells (Fig. 7b). Furthermore, we found that adding recombinant FGF7 to our organoid culture caused a significant increase in alveolar organoid-forming efficiency and size (Fig. 7c). These data highlight the importance of the STAT3-BDNF-TrkB axis to promote regeneration, at least in part, by mediating the release of FGF7 by mesenchymal niche cells (Fig. 7d).

A TrkB agonist improves lung histology following lung injury. To determine whether $\operatorname{TrkB}$ agonists alter lung repair after injury, we administered the $\operatorname{TrkB}$ agonist 7,8-dihydroxyflavone $(7,8-\mathrm{DHF})^{58}$ to mice that underwent acid-induced lung injury. We chose a 2 -d time point to increase the resolution of post-injury recovery in this model with rapid recovery ${ }^{39}$. 7,8-DHF attenuated the degree of histological injury (Fig. 8a) and BAL protein levels $2 \mathrm{~d}$ after injury (Fig. $8 \mathrm{~b}$ ) without altering IL-1 $\beta$ levels (Fig. 8c). We also observed increased AT2 cell proliferation $24 \mathrm{~h}$ after injury and improved alveolar wall thickness $2 \mathrm{~d}$ after acid-induced lung injury (Fig. 8d-f).

Since our model of acid-induced injury is rapidly repaired ${ }^{39}$, the effect of 7,8-DHF treatment to accelerate repair is likely to be inherently modest. To determine whether 7,8-DHF might affect disease characterized by continued injury and repair, we tested the effect of 7,8-DHF on influenza-induced injury. Histological analysis of lungs 21 d post-infection revealed markedly improved architecture (Fig. $8 \mathrm{~g}$ ) and increased AT1 cells (Fig. 8h).

Since the presence of $\mathrm{Krt5}^{+}$pods is known to negatively impact the repair of severely damaged lung tissue ${ }^{27,30}$, we hypothesized that 7,8-DHF would enhance AT2-mediated repair and decrease dysmorphic responses. Quantitative histology of whole-lung sections revealed a significant decrease in $\mathrm{Krt}^{+}$pods in 7,8-DHF-treated murine tissue relative to controls (Fig. 8i,j). Interestingly, 7,8-DHF was unable to rescue influenza-exposed mice that lacked AT2-specific Stat3 (Extended Data Fig. 10), suggesting that other
STAT3 targets, such as cyclin D1, may also be important for the regenerative response to lung injury. These data demonstrate that 7,8-DHF, which pharmacologically enhances TrkB activation, is therefore a pathway to consider in the development of treatments for enhancing recovery from sterile and infectious lung injuries.

\section{Discussion}

The repair of lung tissue has long been known to underlie the resolution of several devastating diseases for which no pharmacological therapies exist ${ }^{9,15,59-62}$. These principles have become increasingly important in light of the current severe acute respiratory syndrome coronavirus 2 pandemic that causes severe acute respiratory distress syndrome in some but not all patients ${ }^{12,13}$. During lung repair, the AT2 cell functions as a progenitor for new AT2 and AT1 cells ${ }^{16-19}$. We hypothesized that genome-wide changes in chromatin accessibility in AT2 cells following acute lung injury associated with transcriptomic analyses of AT2 and mesenchymal niche cells would yield important insights into distal alveolar epithelium regeneration. Here, we show that these analyses converge on a single important cell signalling pathway. We propose that STAT3 activation induces the expression of $B d n f$, which acts through the receptor TrkB to increase mesenchymal expression of Fgf7 and support epithelial regeneration. Our data suggest that targeting of this pathway with the TrkB agonist 7,8-DHF should be examined as a strategy for enhancing the resolution of destructive lung diseases.

We identified changes in AT2 chromatin structure following acute lung injury using ATAC-seq on AT2 cells isolated from control mice compared with mice $24 \mathrm{~h}$ after sustaining acid-induced lung injury. The importance of these changes to alveolar regeneration was suggested by unbiased pathways analysis that classified genes within newly accessible chromatin as supporting regenerative processes. We focused on STAT3 because of our previous data implicating STAT3 activators in alveolar epithelial regeneration ${ }^{39,46}$ and because an unbiased pathway analysis of our ATAC-seq data identified that genes adjacent to newly accessible STAT3 binding sites are involved in regenerative pathways. Although mouse models have previously shown that STAT3 is important for maintaining alveolar epithelium following hyperoxia exposure ${ }^{63}$ and for host defence following Escherichia coli pneumonia ${ }^{64}$, none have specifically interrogated the function of STAT3 in alveolar epithelial regeneration. As such, no STAT3 target genes had yet been implicated in alveolar epithelial regeneration. Here, we demonstrate that changes in chromatin accessibility following acute lung injury portend a second function for STAT3 in the later phases of regeneration. It is important to note that, as shown in Fig. 2b, STAT3 is activated in cell types other than AT2 cells ${ }^{65-67}$. Although we did not identify STAT3 binding sites near known STAT3 activators, we cannot rule

Fig. 4 | Identification of BDNF as important for alveolar epithelial regeneration. a, AT2 cells were isolated from an uninjured C57BL/6 mouse and from a $\mathrm{C} 57 \mathrm{BL} / 6$ mouse that underwent acid-induced lung injury $24 \mathrm{~h}$ previously. Single-cell transcriptomic analysis was performed and cells were grouped into populations using t-distributed stochastic neighbour embedding (t-SNE). There was little overlap between AT2 cells from uninjured and injured mice. b, $K$-means analysis was used to subdivide AT2 cells into groups based on their individual gene expression. Only groups 1, 2, and 3 are indicated, as the others have not been fully investigated. c, Genes whose expression was unique to each cluster are shown. Cluster 3 has gene expression from both AT2 and AT1 cells (Sftpc and Pdpn) and the AEP marker Tm4sf1. d, Bdnf is the only bona fide STAT3 target gene with newly accessible chromatin following acid-induced lung injury that is differentially expressed in cluster 3. e, Data from ATAC-seq (shown in Fig. 1) demonstrate increased accessibility of the

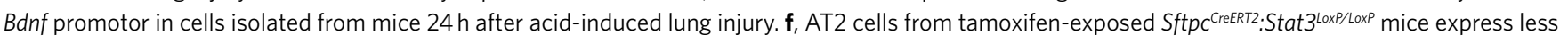
Bdnf than vehicle-exposed mice ( $n=4$ mice per group). $\mathbf{g}$, Analysis of BAL fluid from Sftpc ${ }^{\text {CreERT2}}$ :Stat $3^{\text {LoxP/LXPP }}$ mice treated with either tamoxifen or corn oil (vehicle) before undergoing acid-induced lung injury showed that mice lacking AT2-specific Stat 3 have significantly less BDNF in their BAL fluid ( $n=3$ mice per group per time point). h, AT2 cells from Sftpc CreeRT2-Rosa26TdTomato:Hopx ${ }^{3 F l a g G F P}$ mice were co-cultured with PDGFR $\alpha^{+}$mesenchymal cells from C57BL/6 mice in the presence of BDNF, which increased the alveolar organoid-forming efficiency of murine AT2 cells. The graphs to the right show quantification from $n=3$ distinct cultures per condition. i, Primary human AT2 cells were isolated and co-cultured with MRC 5 fibroblasts for 3 weeks. We observed increased organoid size and forming efficiency when BDNF was added to the culture media. The graphs to the right show the average organoid-forming efficiency and size from $n=3$ different donors. Individual points are shown. In $\mathbf{f - i}$, data are shown as means \pm s.e.m. Statistical significance was determined by two-tailed Student's $t$-test ( $\mathbf{f}, \mathbf{h}$ and $\mathbf{i}$ ) and two-way ANOVA ( $(\mathbf{g})$. 
out the possibility that STAT3 activity in non-AT2 cells is altered by the loss of STAT3 in AT2 cells.

Having identified the importance of STAT3 to alveolar epithelial regeneration, we next sought to identify important effectors using scRNA-seq. We identified a unique cluster of cells-the transdifferentiating population-that emerge $24 \mathrm{~h}$ after acid-induced lung injury and expresses Tm4sf1, the AT2 marker $S f t p c$ and the AT1 marker $P d p n$, despite having been selected against PDPN during isolation. By integrating ATAC-seq and scRNA-seq data, we identified $B d n f$ as the single gene that had a newly accessible STAT3 binding motif and was also differentially upregulated in these transdifferentiating AT2 cells. It is possible that, using different selection a

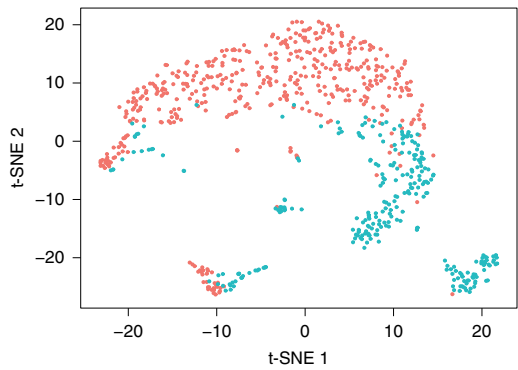

b

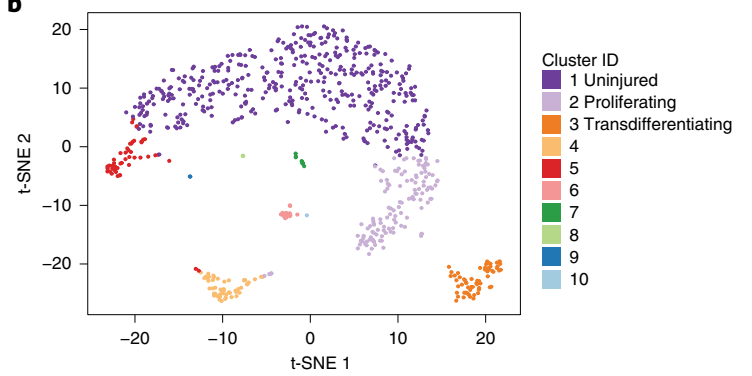

d

$\begin{array}{cc}\text { Genes with } & \text { Genes } \\ \text { newly accessible } & \text { differntially }\end{array}$ STAT3-binding enriched in

sites

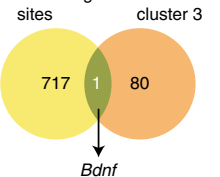

f
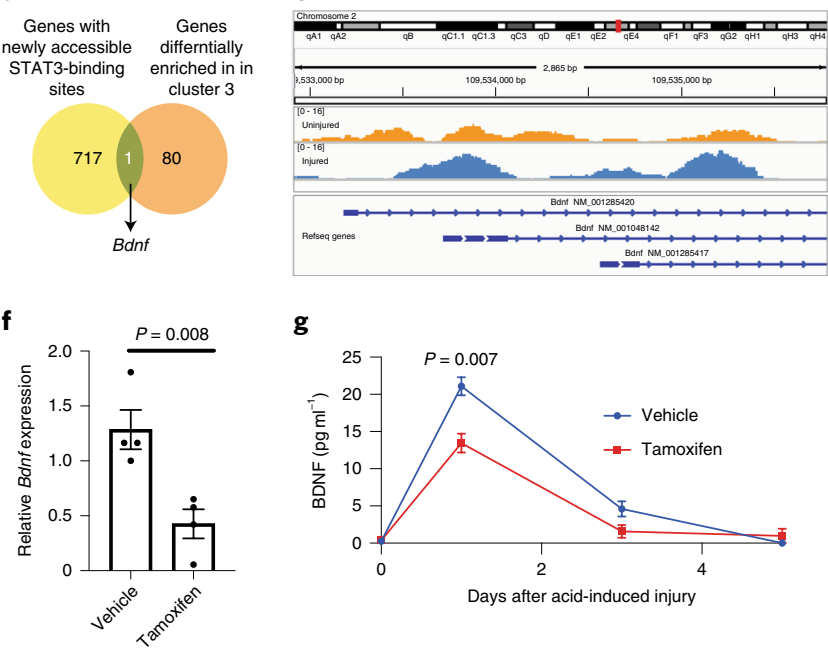

$\mathbf{g}$

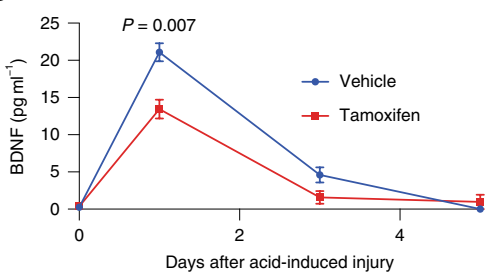

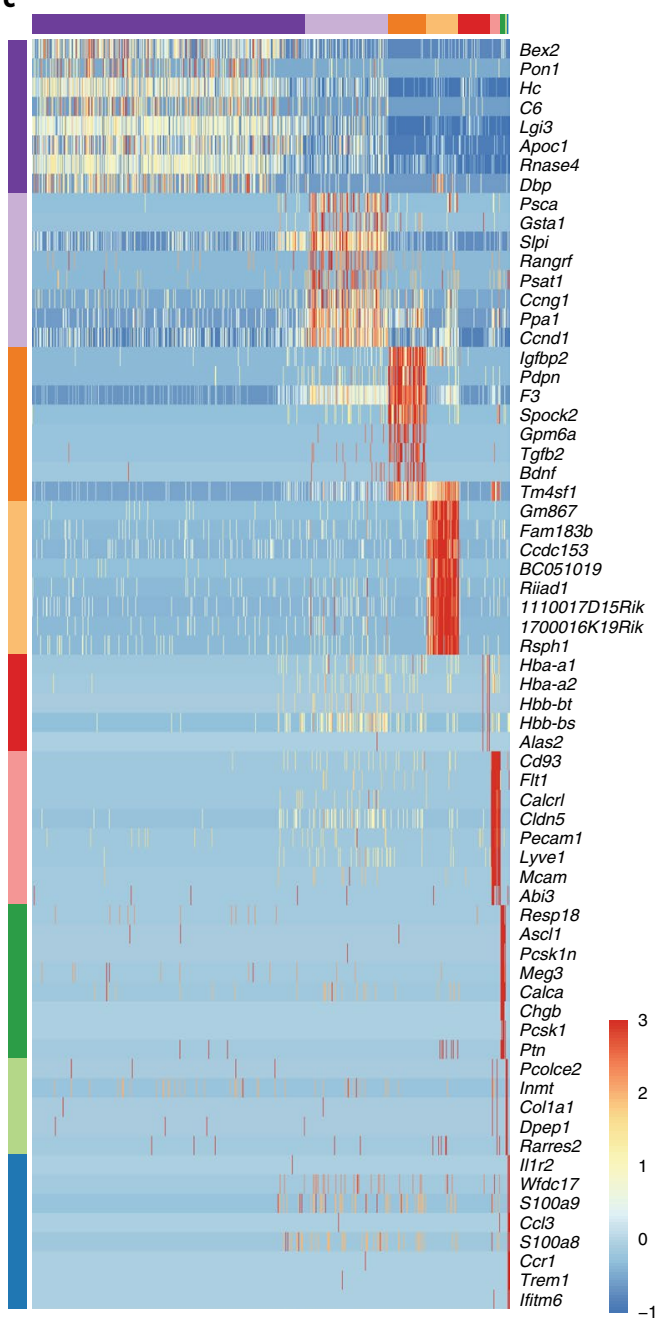

h
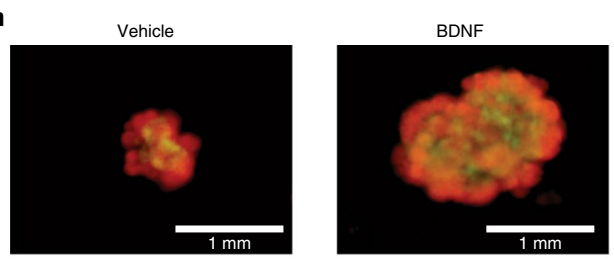

i

Vehicle

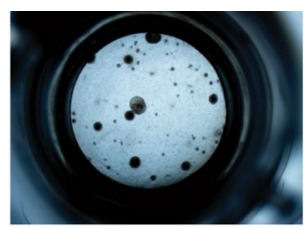

BDNF
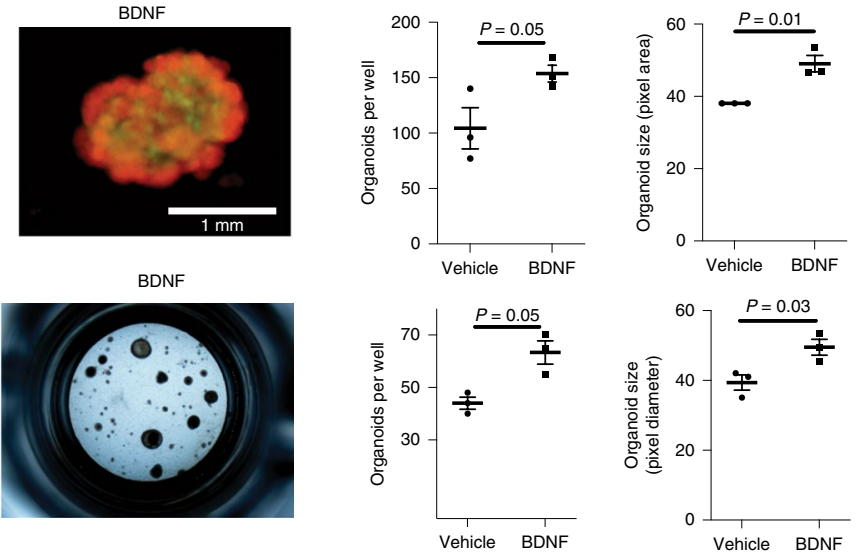


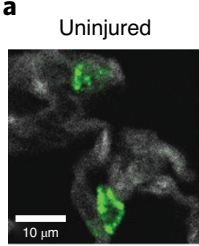

$24 \mathrm{~h}$ post-acid
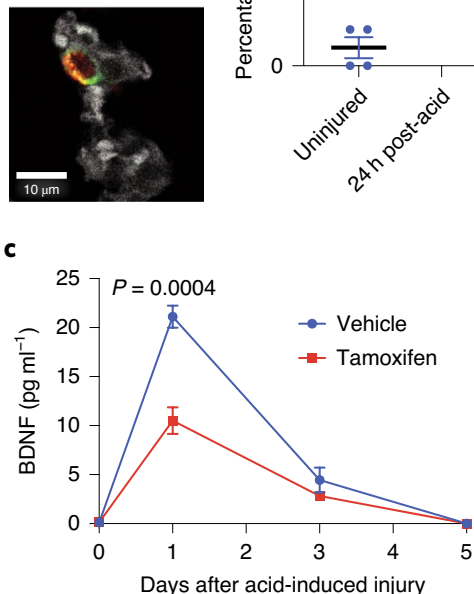

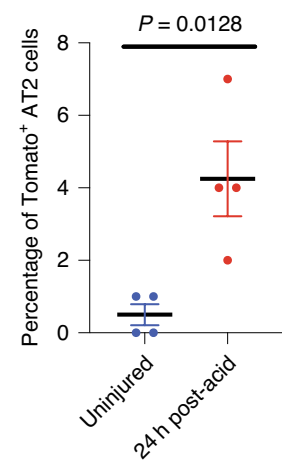

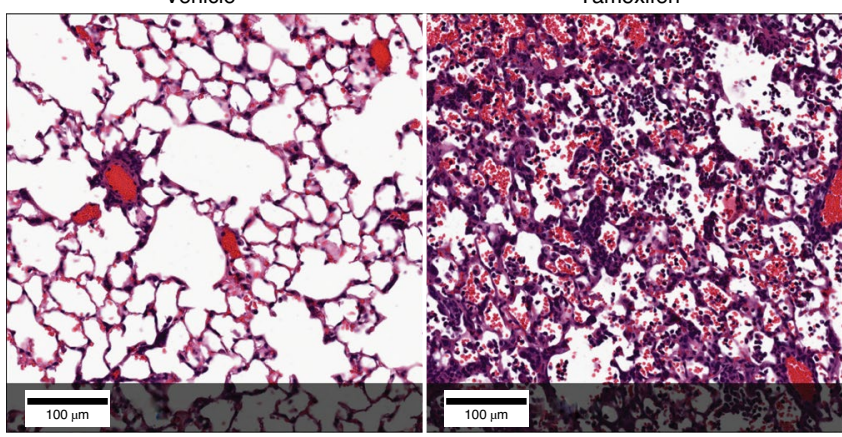

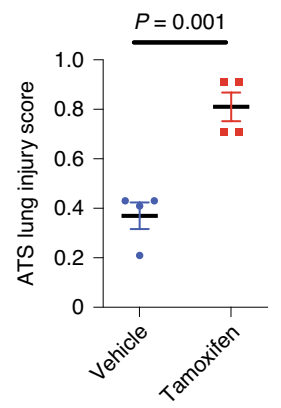

d

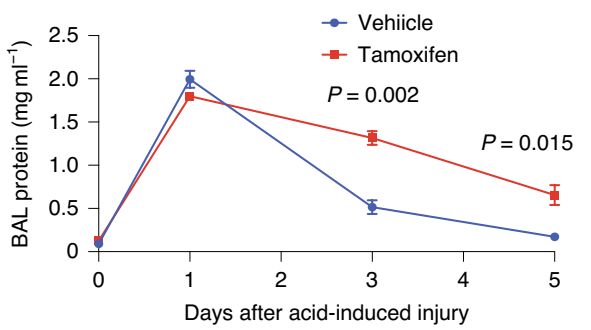

e
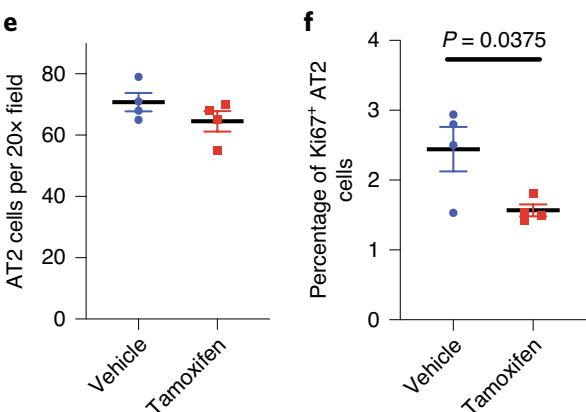

g
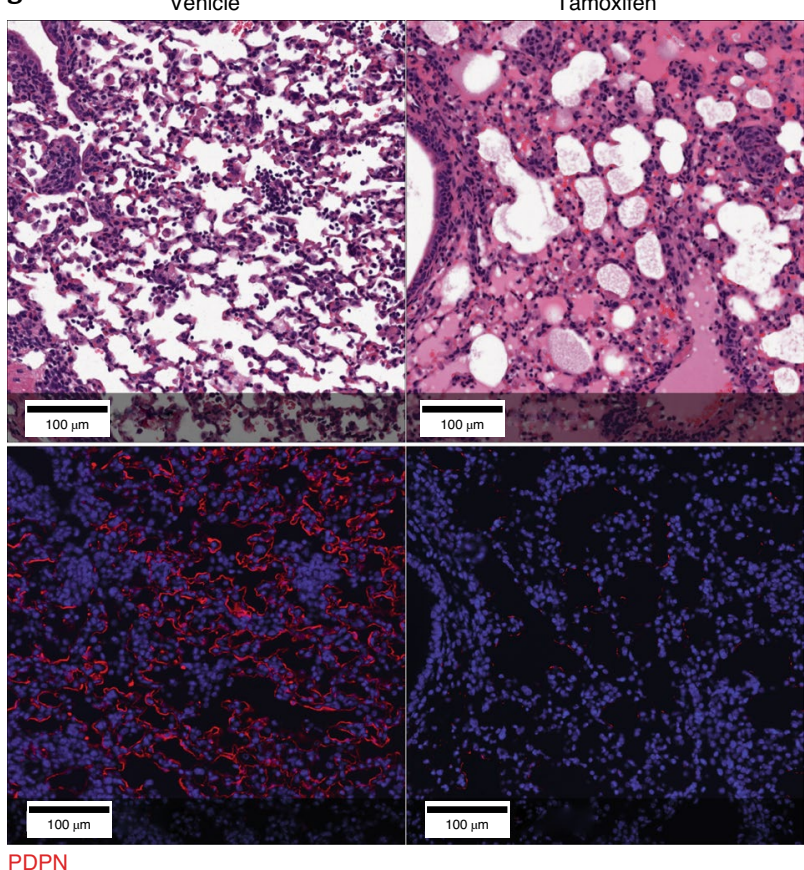

h
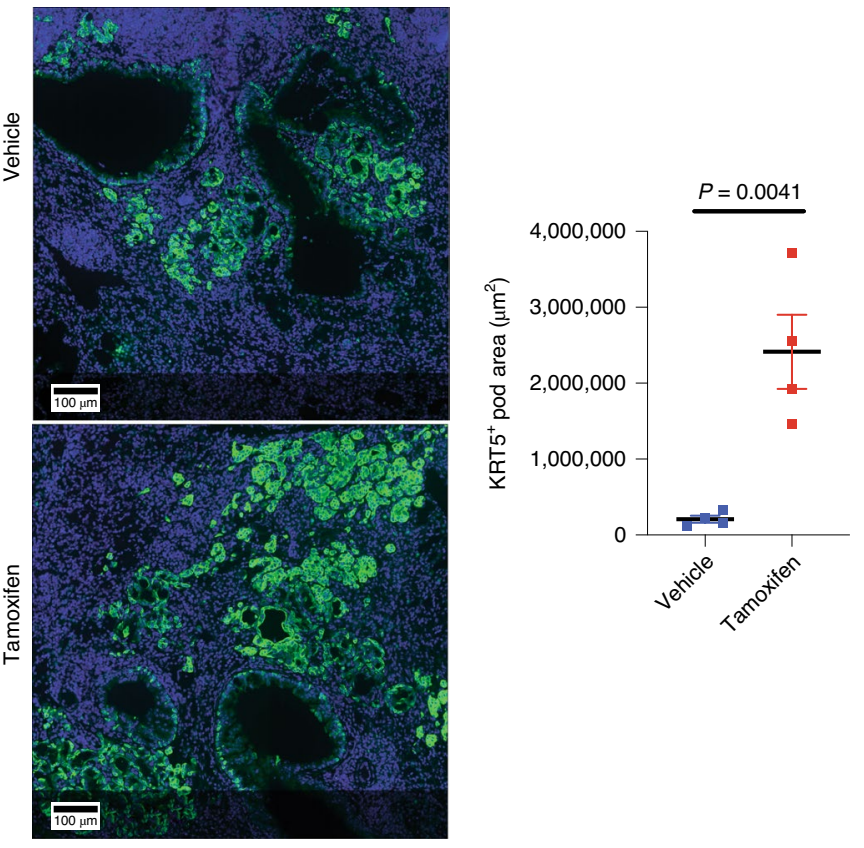

Fig. 5 | Loss of AT2-specific BDNF worsens outcomes following sterile and infectious lung injuries. a, We quantified Tomato ${ }^{+}$AT2 cells in BdnfCre-R26TdTomao mice before and $24 \mathrm{~h}$ after acid-induced lung injury and found a significant increase in co-positive cells following acute lung injury ( $n=4$ mice per group). b-f, Haematoxylin and eosin staining and ATS lung injury scores (b), BAL BDNF concentrations (c), BAL protein concentrations (d), AT2 cell numbers (e) and numbers of proliferating AT2 cells (f) of tamoxifen- and vehicle-exposed Sftpc ${ }^{\text {CreERT2}}{ }^{\text {:BdnfloxP/LoxP }}$ mice $5 \mathrm{~d}$ after acid-included lung injury ( $n=3$ mice per group per time point for $\mathbf{c}$ and $\mathbf{d} ; n=4$ mice per group per time point for $\mathbf{b}, \mathbf{e}$ and $\mathbf{f})$. $\mathbf{g}$, Haematoxylin and eosin and PDPN staining of lung tissue from tamoxifen- and vehicle-exposed Sftpc ${ }^{\text {CreERT2}}: B d n f^{\text {LoxP/LoxP }}$ mice that had been infected with intranasal PR 8 influenza $\left(5 \times 10^{-5} \mathrm{HAU}\right.$ per mouse) $(n=4$ mice per group). Images were taken at $21 \mathrm{~d}$ post-infection. $\mathbf{h}$, KRT5 staining of the mice described in $\mathbf{g}$, with quantification of KRT5+ pods in both groups ( $n=4$ mice per group). Analysis was conducted at $21 \mathrm{~d}$ post-infection. In a-f and $\mathbf{h}$, data are shown as means \pm s.e.m. Statistical significance was determined by two-tailed Student's t-test ( $\mathbf{a}, \mathbf{b}, \mathbf{e}, \mathbf{f}$ and $\mathbf{h}$ ) and two-way ANOVA (c and $\mathbf{d}$ ).

criteria, we would have identified additional candidates. Although BDNF stands for 'brain-derived neurotrophic factor', the regulation of $B d n f$ by STAT3 has previously been reported in multiple cell types $^{53-56}$. While BDNF has been detected in lung cancer lines ${ }^{68}$, it has not previously been implicated in alveolar epithelial regeneration. The importance of BDNF for alveolar epithelial regeneration 
a

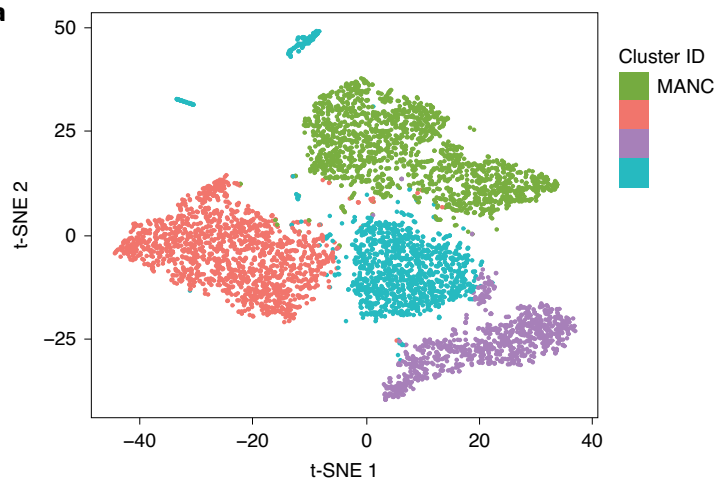

c

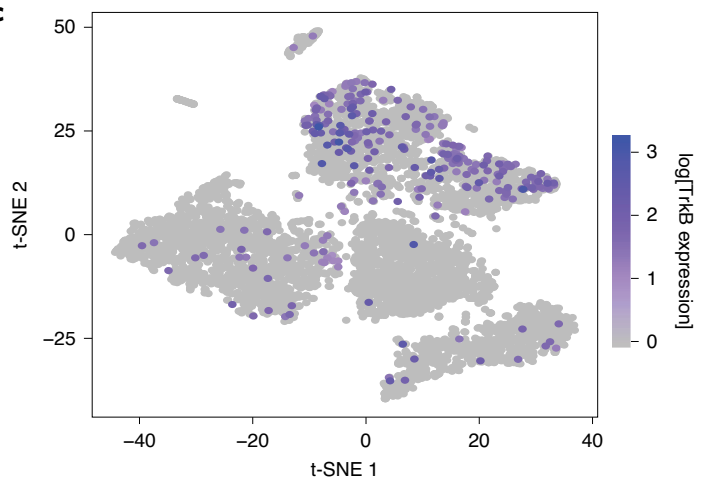

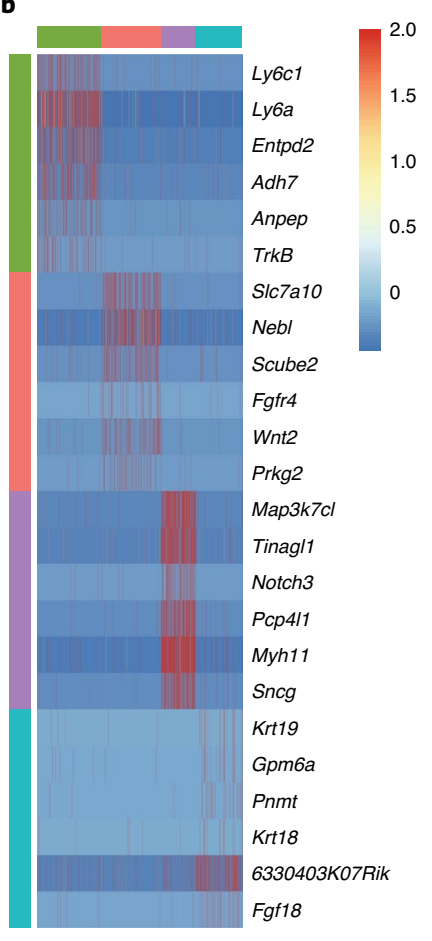

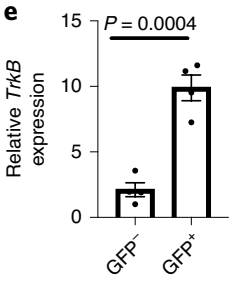

$-\log [P$ value $]$

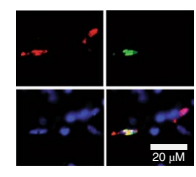

PDGFR $\alpha$ GFP DAPI

j

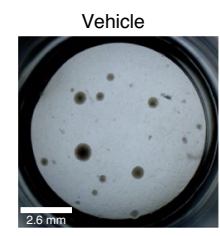

g
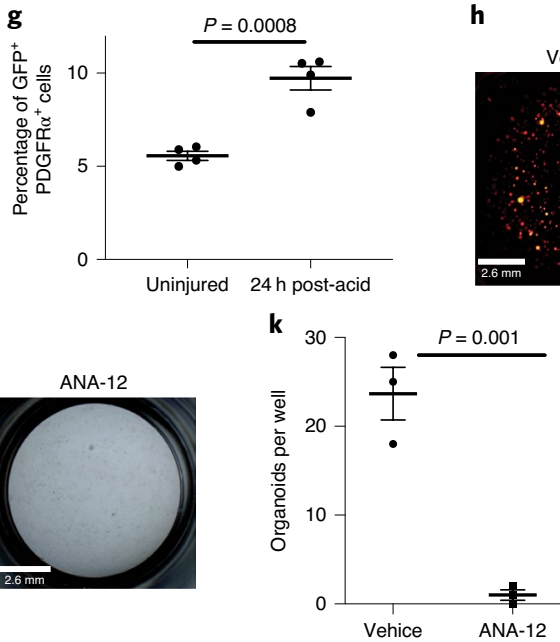

Vehicle
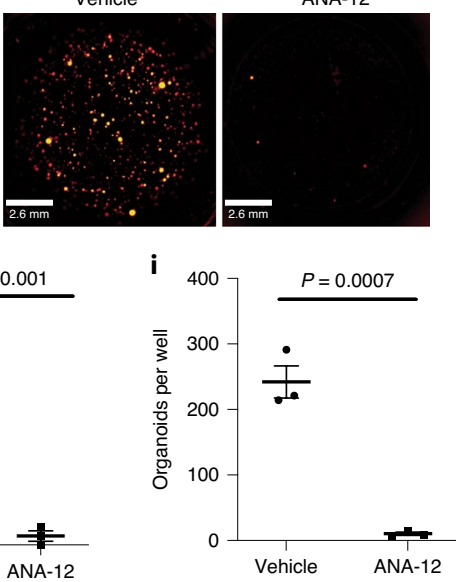

Fig. 6 | BDNF-TrkB signalling promotes alveolar epithelial regeneration. a-c, t-SNE analysis of cluster ID (a) and log[TrkB expression] (c), along with gene expression analysis by cluster (b) following scRNA-seq of mesenchymal cells from uninjured mice showed that TrkB expression is enriched in MANCs. Other mesenchymal populations in a have not been rigorously defined, so are unlabeled. $\mathbf{d}$, Ingenuity pathways analysis showed that mesenchymal cells expressing $\operatorname{Trk} B$ are enriched with the expression of genes that control respiratory system development, organ development and tissue morphology. e, Expression of $T r k B$ in green fluorescent protein-positive (GFP+) and GFP- cells from TrkB EGFP mice ( $n=4$ mice per group). $\mathbf{f}$, Expression of TrkB on mesenchymal cells was verified by staining lung tissue from TrkBEGFP mice for PDGFR $\alpha$. $\mathbf{g}$, Quantification of GFP and PDGFR $\alpha$ co-positive cells in TrkB ${ }^{E G F P}$ mice $24 \mathrm{~h}$ after acid-induced lung injury ( $n=4$ mice per group). The gating strategy is shown in Extended Data Fig. 8. h,i, AT2 cells isolated from a tamoxifen-treated Sftpc CreeRT2-Rosa26mTmG mouse were co-cultured with PDGFR $\alpha^{+}$mesenchymal cells for 4 weeks. Images (h) and quantification (i) show that ANA-12 abrogated the organoid-forming efficiency of AT2 cells ( $n=3$ wells per condition). $\mathbf{j}, \mathbf{k}$, Images (j) and quantification ( $\mathbf{k}$ ) show that the organoid-forming capacity of primary human AT2 cells was abrogated by the addition of ANA-12 to the media. Cultures were grown for 4 weeks. In $\mathbf{i}$ and $\mathbf{k}, n=3$ different donors. In $\mathbf{e}, \mathbf{g}, \mathbf{i}$ and $\mathbf{k}$, data are shown as means \pm s.e.m. Statistical significance was determined by two-tailed Student's $t$-test $(\mathbf{e}, \mathbf{g}, \mathbf{i}$ and $\mathbf{k})$. 
a

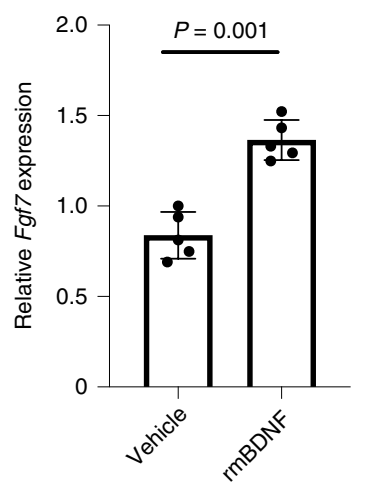

b

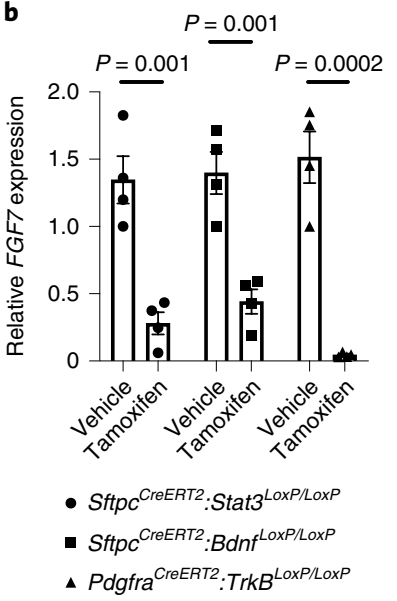

c

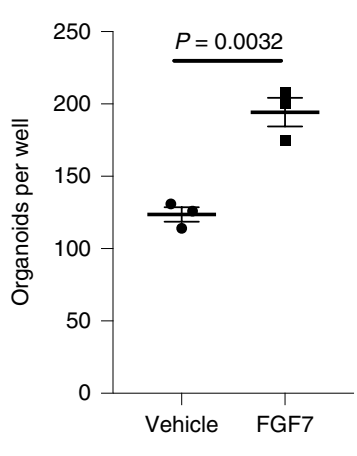

Vehicle

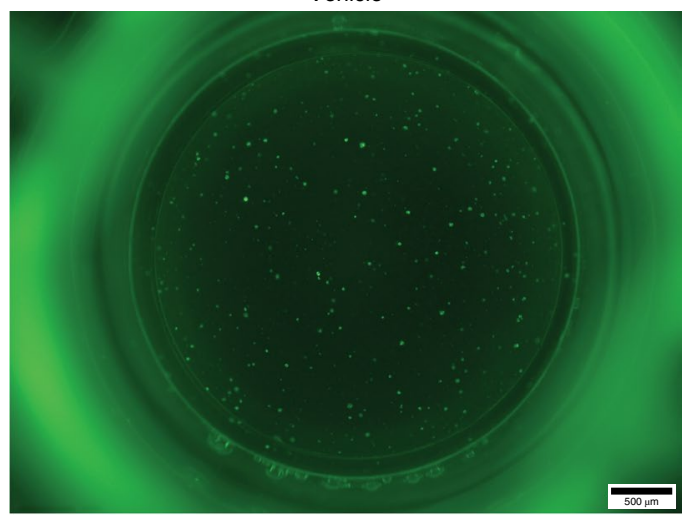

FGF7
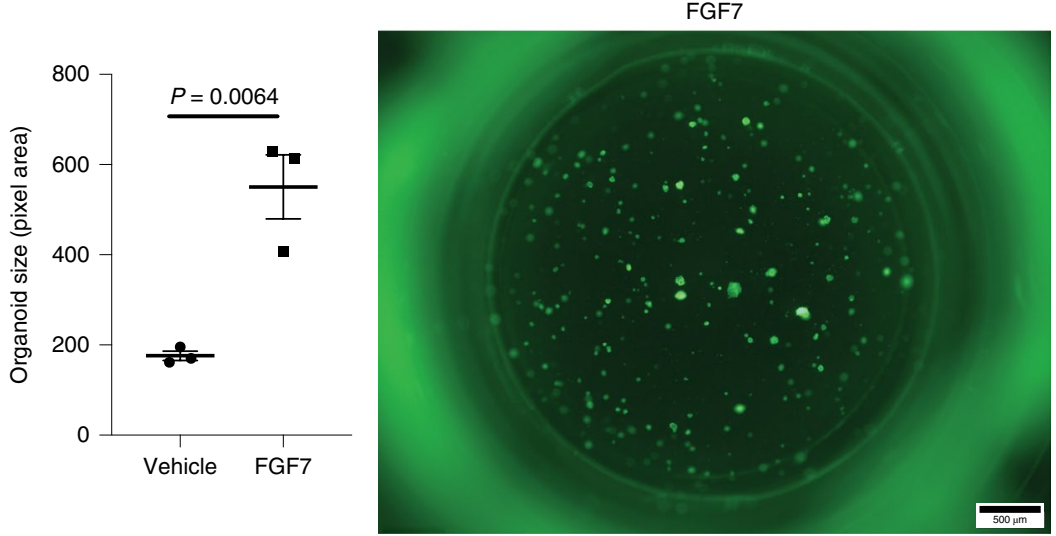

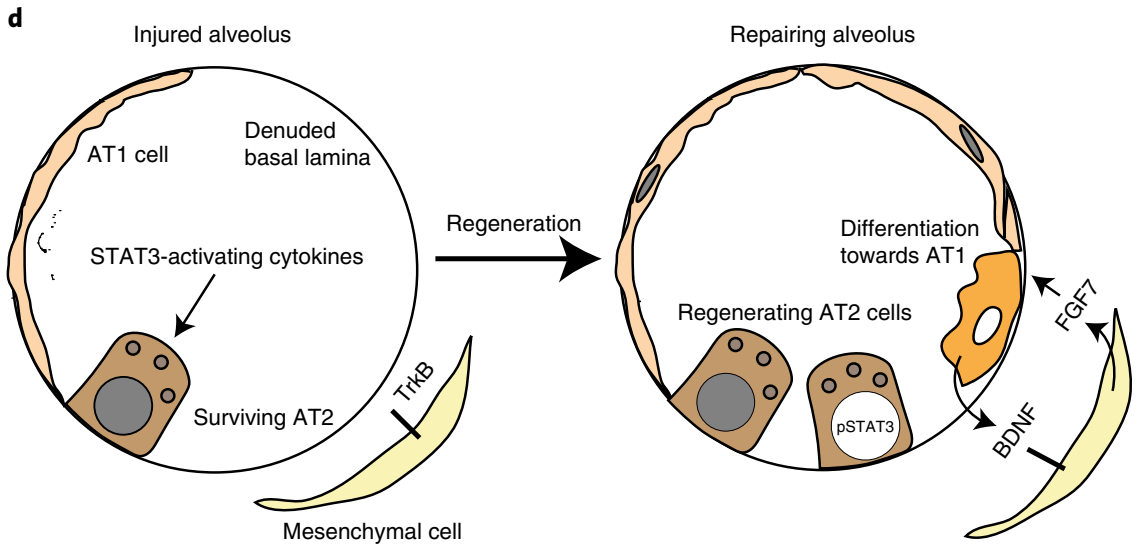

Fig. 7 | The STAT3-BDNF-TrkB axis modulates Fgf7 expression by mesenchymal niche cells. a, Fgf7 messenger RNA expression in mesenchymal cells isolated from the organoid culture conditions described in Fig. $4 \mathrm{~h}$ ( $n=5$ wells per condition). b, Fgf7 expression in PDGFR $\alpha^{+}$cells isolated from tamoxifen-

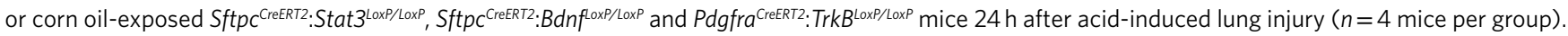
c, The addition of $0.1 \mu \mathrm{g} \mathrm{ml}^{-1}$ recombinant murine BDNF caused a significant increase in alveolar organoid-forming efficiency and size ( $n=3$ wells per condition). $\mathbf{d}$, In the setting of acute lung injury, STAT3-activating cytokines accumulate in the alveolus and STAT3 becomes activated in the AT2 cell. Accompanying changes in chromatin accessibility within AT2 cells allow for the expression of BDNF in transdifferentiating cells, which then binds to TrkB on mesenchymal niche cells to stimulate the expression of Fgf7, which promotes alveolar epithelial regeneration. In a-c, data are shown as means \pm s.e.m. Statistical significance was determined by two-tailed Student's $t$-test (a-c).

was confirmed using organoids-a simplified model of alveolar development that effectively isolated the roles of epithelial and mesenchymal cells. Adding recombinant human BDNF to the organoid media significantly increased organoid size and the forming efficiency of primary murine and human AT2 cells. Although we identified BDNF by isolating AT2 cells, its expression is seen only in transdifferentiating AT2 cells; thus, we cannot resolve whether this upregulation is part of the AT2 to AT1 transition or unique to the reparative state.

To understand the underlying cell signalling mechanism through which BDNF promotes alveolar repair, we sought to identify which cell types express $\operatorname{TrkB}$, the cognate receptor for $\mathrm{BDNF}^{69}$. Analysis of 
a

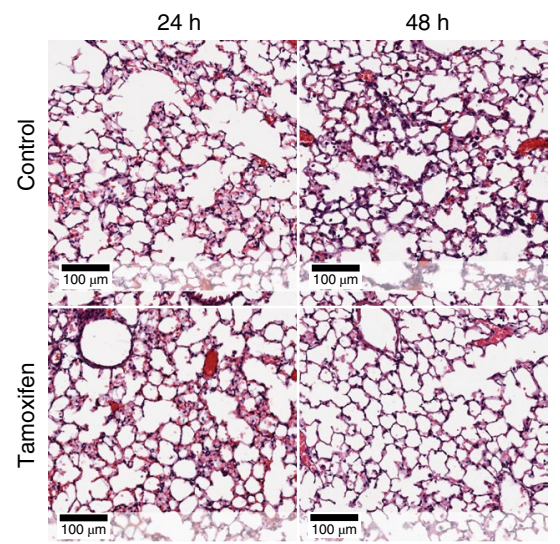

d

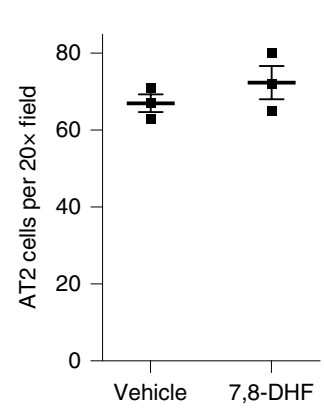

h

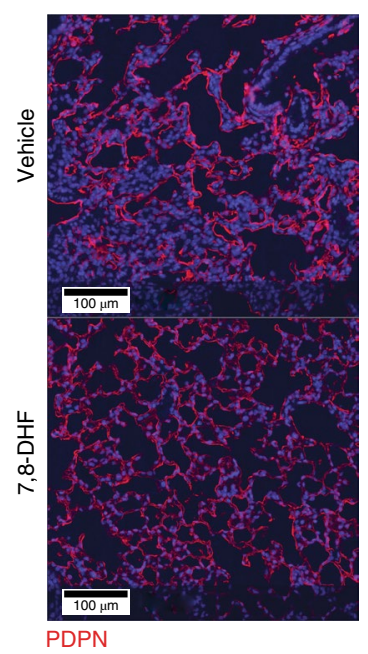

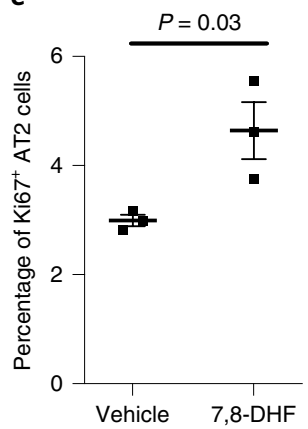

b
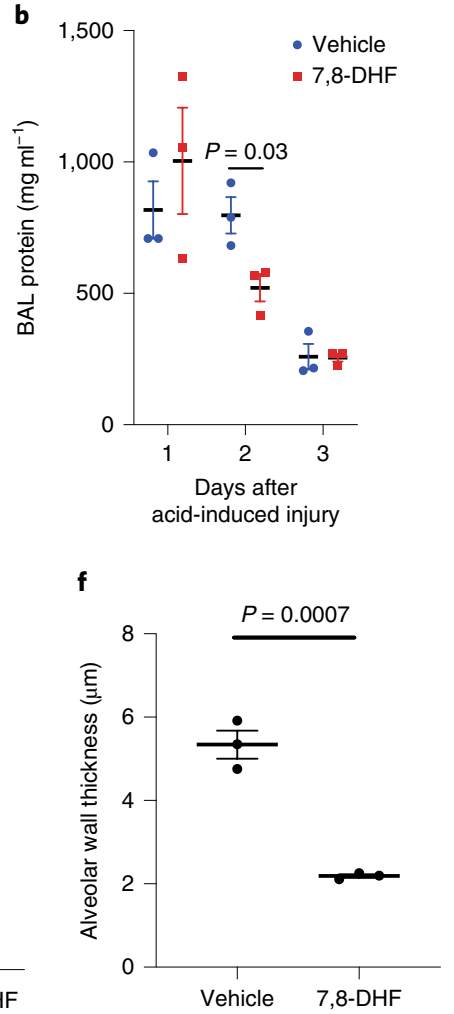

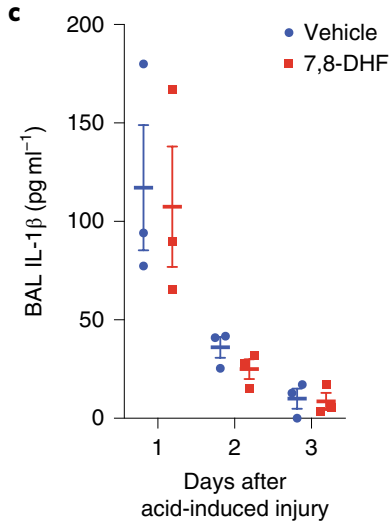

g

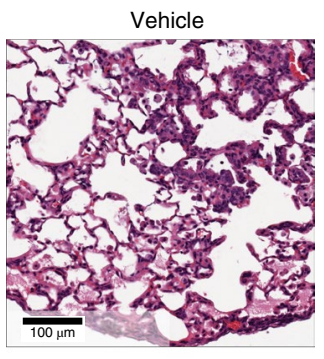

7,8-DHF

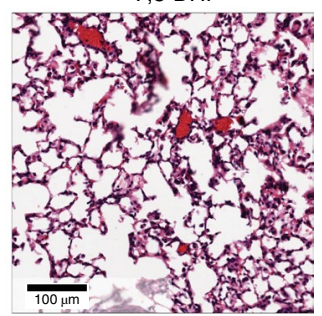

j

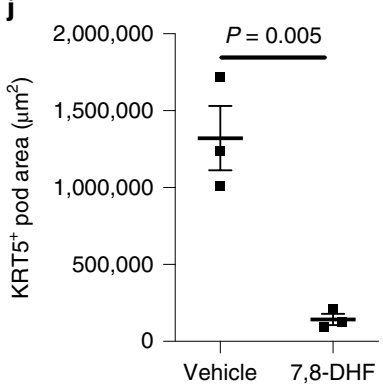

Fig. 8 | The TrkB agonist 7,8-DHF improves outcomes following sterile and infectious lung injury. a-e, C57BL/6 mice underwent acid-induced unilateral lung injury and were given an intraperitoneal injection of 7,8-DHF or vehicle control at the time of injury ( $n=3$ mice per group). Mice that were euthanized at $48 \mathrm{~h}$ received an additional intraperitoneal dose of 7,8-DHF or vehicle $24 \mathrm{~h}$ after undergoing acid-induced lung injury. Mice that received 7,8 -DHF had improved lung histology (a), significantly less BAL protein (b) and increased numbers of AT 2 cells per $20 \times$ field (d) and percentages of Ki67+ AT2 cells (e), indicating increased AT2 cell proliferation, at $48 \mathrm{~h}$ after lung injury. No differences in IL-1 $\beta$ (c) were observed. f, Alveolar wall thickness, measured using ten $20 \times$ images per lung (means of $n=3$ mice per group). $\mathbf{g}$,h, Haematoxylin and eosin (g) and PDPN staining (h) of lung tissue from C57BL/6 mice infected with intranasal PR8 influenza $\left(5 \times 10^{-5} \mathrm{HAU}\right.$ per mouse). Intraperitoneal injections of 7,8-DHF or vehicle were administered every other day $(n=3$ mice per group). $\mathbf{i}, \mathbf{j}$, Images (i) and quantification (j) of KRT5+ pods from the mice described in $\mathbf{g}$ and $\mathbf{h}$ (means of $n=3$ mice per group). In $\mathbf{g}-\mathbf{i}$, images were captured at $21 \mathrm{~d}$ post-infection. In $\mathbf{i}$, the images to the right are magnified versions of the parts outlined in by the white boxes to the left. In $\mathbf{b}-\mathbf{f}$ and $\mathbf{j}$, data are shown as means \pm s.e.m. Statistical significance was determined by two-tailed Student's $t$-test at each time point shown in $\mathbf{b}-\mathbf{f}$ and $\mathbf{j}$.

pulmonary mesenchymal cells using scRNA-seq indicated that TrkB expression was uniquely enriched in the MANC population, which expands during regeneration ${ }^{46}$. Likewise, we found that the number of TrkB and PDGFR $\alpha$ co-positive cells significantly increased following acute lung injury, further suggesting the importance of
BDNF-responsive niche cells as a part of the regenerative response. Adding the TrkB antagonist ANA-12 to organoid media abrogated alveolar organoid formation, demonstrating that TrkB signalling is necessary for alveolar organoid development. These data suggest that activating the $\operatorname{TrkB}$ receptor could be beneficial to recovery 
from acute lung injury. Administering the recently developed TrkB agonist 7,8-DHF ${ }^{58}$ led to faster recovery from acid-induced injury and H1N1 influenza infection. While we implicated a role for FGF7, further work is necessary to determine the impact of BDNF and TrkB agonists during homeostasis and the regeneration on FGF7 as well as other potential mediators. Further studies are needed to see if the impact of 7,8-DHF on outcomes following lung injury is due to differences in inflammation or regeneration alone. Our studies using BDNF and ANA-12 in vitro strongly suggest that at least part of the effect we observed with 7,8-DHF treatment was due to increased alveolar epithelial regeneration.

In summary, these data show that effective regeneration of the alveolar epithelium requires bidirectional communication between epithelial and mesenchymal cells. In the epithelial cells, our data show that activation of STAT3 in AT2 cells plays a central role in repair. At least one of the downstream effects of STAT3 activation appears to be induction of $B d n f$, which activates TrkB-expressing mesenchymal niche cells to modulate the expression of $\mathrm{Fgf7}$, which subsequently interacts with epithelial cells to play an essential regenerative role (Fig. 8d). From a translational standpoint, this process can be enhanced pharmacologically by administration of a TrkB agonist. Future studies are needed to elucidate how chromosomal architecture is regulated in AT2 cells after acute lung injury, how STAT3 becomes activated in AT2 cells, and which other cells may respond to BDNF in vivo. Nevertheless, the contribution of the STAT3-BDNF-TrkB axis in orchestrating alveolar epithelial regeneration might open up opportunities for drug discovery, precision targets and therapeutic interventions.

\section{Online content}

Any methods, additional references, Nature Research reporting summaries, source data, extended data, supplementary information, acknowledgements, peer review information; details of author contributions and competing interests; and statements of data and code availability are available at https://doi.org/10.1038/ s41556-020-0569-x.

Received: 19 June 2019; Accepted: 3 August 2020;

Published online: 28 September 2020

\section{References}

1. Matthay, M. A., Robriquet, L. \& Fang, X. Alveolar epithelium: role in lung fluid balance and acute lung injury. Proc. Am. Thorac. Soc. 2, 206-213 (2005).

2. Guillot, L. et al. Alveolar epithelial cells: master regulators of lung homeostasis. Int. J. Biochem. Cell Biol. 45, 2568-2573 (2013).

3. Tyrrell, C., McKechnie, S. R., Beers, M. F., Mitchell, T. J. \& McElroy, M. C. Differential alveolar epithelial injury and protein expression in pneumococcal pneumonia. Exp. Lung Res. 38, 266-276 (2012).

4. Herold, S., Becker, C., Ridge, K. M. \& Budinger, G. R. Influenza virus-induced lung injury: pathogenesis and implications for treatment. Eur. Respir. J. 45, 1463-1478 (2015)

5. Herold, S. et al. Lung epithelial apoptosis in influenza virus pneumonia: the role of macrophage-expressed TNF-related apoptosis-inducing ligand. J. Exp. Med. 205, 3065-3077 (2008).

6. Matthay, M. A., Ware, L. B. \& Zimmerman, G. A. The acute respiratory distress syndrome. J. Clin. Invest. 122, 2731-2740 (2012).

7. Thompson, B. T., Chambers, R. C. \& Liu, K. D. Acute respiratory distress syndrome. N. Engl. J. Med. 377, 1904-1905 (2017).

8. Martin, T. R., Hagimoto, N., Nakamura, M. \& Matute-Bello, G. Apoptosis and epithelial injury in the lungs. Proc. Am. Thorac. Soc. 2, 214-220 (2005).

9. Herridge, M. S. et al. One-year outcomes in survivors of the acute respiratory distress syndrome. N. Engl. J. Med. 348, 683-693 (2003).

10. Matthay, M. A. \& Wiener-Kronish, J. P. Intact epithelial barrier function is critical for the resolution of alveolar edema in humans. Am. Rev. Resp. Dis. 142, 1250-1257 (1990).

11. Ingbar, D. H. \& Matthay, R. A. Pulmonary sequelae and lung repair in survivors of the adult respiratory distress syndrome. Crit. Care Clin. 2, 629-665 (1986).

12. Guan, W. et al. Clinical characteristics of coronavirus disease 2019 in China. N. Engl. J. Med. 382, 1708-1720 (2020).
13. Bhatraju, P. K. et al. Covid-19 in critically ill patients in the Seattle region-case series.N. Engl. J. Med. 382, 2012-2022(2020).

14. Konigshoff, M., Saglani, S., Marsland, B. J. \& Eickelberg, O. Rebuilding a diseased lung: repair and regeneration. Eur. Respir. J. 41, 497-499 (2013).

15. Kotton, D. N. \& Morrisey, E. E. Lung regeneration: mechanisms, applications and emerging stem cell populations. Nat. Med. 20, 822-832 (2014).

16. Evans, M. J., Cabral, L. J., Stephens, R. J. \& Freeman, G. Renewal of alveolar epithelium in the rat following exposure to $\mathrm{NO}_{2}$. Am. J. Pathol. 70, 175-198 (1973).

17. Barkauskas, C. E. et al. Type 2 alveolar cells are stem cells in adult lung. J. Clin. Invest. 123, 3025-3036 (2013).

18. Nabhan, A. N., Brownfield, D. G., Harbury, P. B., Krasnow, M. A. \& Desai, T. J. Single-cell Wnt signaling niches maintain stemness of alveolar type 2 cells. Science 359, 1118-1123 (2018).

19. Zacharias, W. J. et al. Regeneration of the lung alveolus by an evolutionarily conserved epithelial progenitor. Nature 555, 251-255 (2018).

20. Jain, R. et al. Plasticity of $\mathrm{Hopx}^{+}$type I alveolar cells to regenerate type II cells in the lung. Nat. Commun. 6, 6727 (2015).

21. Wang, Y. et al. Pulmonary alveolar type I cell population consists of two distinct subtypes that differ in cell fate. Proc. Natl Acad. Sci. USA 115, 2407-2412 (2018).

22. Kim, C. F. et al. Identification of bronchioalveolar stem cells in normal lung and lung cancer. Cell 121, 823-835 (2005).

23. Liu, Q. et al. Lung regeneration by multipotent stem cells residing at the bronchioalveolar-duct junction. Nat. Genet. 51, 728-738 (2019).

24. Salwig, I. et al. Bronchioalveolar stem cells are a main source for regeneration of distal lung epithelia in vivo.EMBO J. 38, e102099 (2019).

25. Zuo, W. et al. p63+Krt5 ${ }^{+}$distal airway stem cells are essential for lung regeneration. Nature 517, 616-620 (2015).

26. Ray, S. et al. Rare SOX2 ${ }^{+}$airway progenitor cells generate $\mathrm{KRT}^{+}$cells that repopulate damaged alveolar parenchyma following influenza virus infection. Stem Cell Rep. 7, 817-825 (2016).

27. Vaughan, A. E. et al. Lineage-negative progenitors mobilize to regenerate lung epithelium after major injury. Nature 517, 621-625 (2015).

28. Nolen-Walston, R. D. et al. Cellular kinetics and modeling of bronchioalveolar stem cell response during lung regeneration. Am. J. Physiol. Lung Cell. Mol. Physiol. 294, L1158-L1165 (2008).

29. Yee, M. et al. Alternative progenitor lineages regenerate the adult lung depleted of alveolar epithelial type 2 cells. Am. J. Respir. Cell Mol. Biol. 56, 453-464 (2017)

30. Xi, Y. et al. Local lung hypoxia determines epithelial fate decisions during alveolar regeneration. Nat. Cell Biol. 19, 904-914 (2017).

31. Mason, R. J. \& Williams, M. C. Type II alveolar cell. Defender of the alveolus. Am. Rev. Resp. Dis. 115, 81-91 (1977).

32. Fehrenbach, H. Alveolar epithelial type II cell: defender of the alveolus revisited. Respir. Res. 2, 33-46 (2001).

33. Crapo, J. D. et al. Morphometric characteristics of cells in the alveolar region of mammalian lungs. Am. Rev. Resp. Dis. 128, S42-S46 (1983).

34. Takahashi, K. \& Yamanaka, S. Induction of pluripotent stem cells from mouse embryonic and adult fibroblast cultures by defined factors. Cell 126, 663-676 (2006)

35. Rodolfa, K. T. \& Eggan, K. A transcriptional logic for nuclear reprogramming. Cell 126, 652-655 (2006)

36. Buenrostro, J. D., Giresi, P. G., Zaba, L. C., Chang, H. Y. \& Greenleaf, W. J. Transposition of native chromatin for fast and sensitive epigenomic profiling of open chromatin, DNA-binding proteins and nucleosome position. Nat. Methods 10, 1213-1218 (2013).

37. Barkauskas, C. E. et al. Lung organoids: current uses and future promise. Development 144, 986-997 (2017)

38. Kadyk, L. C., DeWitt, N. D. \& Gomperts, B. Proceedings: regenerative medicine for lung diseases: a CIRM Workshop Report. Stem Cells Transl. Med. 6, 1823-1828 (2017).

39. Paris, A. J. et al. Neutrophils promote alveolar epithelial regeneration by enhancing type II pneumocyte proliferation in a model of acid-induced acute lung injury.Am. J. Physiol. Lung Cell. Mol. Physiol. 311, L1062-L1075 (2016).

40. Heinz, S. et al. Simple combinations of lineage-determining transcription factors prime cis-regulatory elements required for macrophage and B cell identities. Mol. Cell 38, 576-589 (2010).

41. Kimura, S. et al. The T/ebp null mouse: thyroid-specific enhancer-binding protein is essential for the organogenesis of the thyroid, lung, ventral forebrain, and pituitary. Genes Dev. 10, 60-69 (1996).

42. Yuan, B. et al. Inhibition of distal lung morphogenesis in $N k x 2.1^{-1-}$ embryos. Dev. Dynam. 217, 180-190 (2000).

43. Li, S. et al. Foxp1/4 control epithelial cell fate during lung development and regeneration through regulation of anterior gradient 2. Development 139, 2500-2509 (2012).

44. Shu, W. et al. Foxp2 and Foxp1 cooperatively regulate lung and esophagus development. Development 134, 1991-2000 (2007). 
45. Ghahary, A. \& Ghaffari, A. Role of keratinocyte-fibroblast cross-talk in development of hypertrophic scar. Wound Repair Regen. 15, S46-S53 (2007).

46. Zepp, J. A. et al. Distinct mesenchymal lineages and niches promote epithelial self-renewal and myofibrogenesis in the lung. Cell 170, 1134-1148.e10 (2017)

47. Schutte, H. et al. Bronchoalveolar and systemic cytokine profiles in patients with ARDS, severe pneumonia and cardiogenic pulmonary oedema. Eur. Respir. J. 9, 1858-1867 (1996).

48. Zhong, Z., Wen, Z. \& Darnell, J. E. Jr Stat3: a STAT family member activated by tyrosine phosphorylation in response to epidermal growth factor and interleukin-6. Science 264, 95-98 (1994).

49. Zemans, R. L. \& Matthay, M. A. Bench-to-bedside review: the role of the alveolar epithelium in the resolution of pulmonary edema in acute lung injury. Crit. Care 8, 469-477 (2004).

50. Matthay, M. A., Folkesson, H. G. \& Clerici, C. Lung epithelial fluid transport and the resolution of pulmonary edema. Physiol. Rev. 82, 569-600 (2002).

51. Riemondy, K. A. et al. Single cell RNA sequencing identifies TGF $\beta$ as a key regenerative cue following LPS-induced lung injury.JCI Insight $\mathbf{5}$, e123637 (2019).

52. Leibrock, J. et al. Molecular cloning and expression of brain-derived neurotrophic factor. Nature 341, 149-152 (1989).

53. Chen, B. et al. Autocrine activity of BDNF induced by the STAT3 signaling pathway causes prolonged TrkB activation and promotes human non-small-cell lung cancer proliferation. Sci. Rep. 6, 30404 (2016).

54. Tang, Q. P. et al. STAT3 signal that mediates the neural plasticity is involved in willed-movement training in focal ischemic rats. J. Zhejiang Univ. Sci. B 17, 493-502 (2016).

55. Zhang, J. X. et al. Unique genome-wide map of TCF4 and STAT3 targets using ChIP-seq reveals their association with new molecular subtypes of glioblastoma. Neuro. Oncol. 15, 279-289 (2013).

56. Hixson, K. M., Cogswell, M., Brooks-Kayal, A. R. \& Russek, S. J. Evidence for a non-canonical JAK/STAT signaling pathway in the synthesis of the brain's major ion channels and neurotransmitter receptors. BMC Genomics $\mathbf{2 0}$, 677 (2019)
57. Cazorla, M. et al. Identification of a low-molecular weight TrkB antagonist with anxiolytic and antidepressant activity in mice. J. Clin. Invest. 121, 1846-1857 (2011).

58. Jang, S. W. et al. A selective TrkB agonist with potent neurotrophic activities by 7,8-dihydroxyflavone. Proc. Natl Acad. Sci. USA 107, 2687-2692 (2010).

59. Hogan, B. Stemming lung disease? N. Engl. J. Med. 378, 2439-2440 (2018).

60. Weiss, D. J. Concise review: current status of stem cells and regenerative medicine in lung biology and diseases. Stem Cells 32, 16-25 (2014).

61. Yang, J. \& Jia, Z. Cell-based therapy in lung regenerative medicine. Regen. Med. Res. 2, 7 (2014).

62. Kang, M. \& Thebaud, B. Stem cell biology and regenerative medicine for neonatal lung diseases. Pediatr. Res. 83, 291-297 (2018).

63. Hokuto, I. et al. Stat-3 is required for pulmonary homeostasis during hyperoxia. J. Clin. Invest. 113, 28-37 (2004).

64. Quinton, L. J. et al. Alveolar epithelial STAT3, IL-6 family cytokines, and host defense during Escherichia coli pneumonia. Am. J. Respir. Cell Mol. Biol. 38, 699-706 (2008).

65. Severgnini, M. et al. Activation of the STAT pathway in acute lung injury. Am. J. Physiol. Lung Cell. Mol. Physiol. 286, L1282-L1292 (2004).

66. Liu, X. STAT3 activation inhibits human bronchial epithelial cell apoptosis in response to cigarette smoke exposure. Biochem. Biophys. Res. Commun. 353, 121-126 (2007).

67. Tadokoro, T. et al. IL-6/STAT3 promotes regeneration of airway ciliated cells from basal stem cells. Proc. Natl Acad. Sci. USA 111, E3641-E3649 (2014).

68. Giard, D. J. et al. In vitro cultivation of human tumors: establishment of cell lines derived from a series of solid tumors. J. Natl Cancer Inst. 51, 1417-1423 (1973).

69. Chao, M. V. Neurotrophins and their receptors: a convergence point for many signalling pathways. Nat. Rev. Neurosci. 4, 299-309 (2003).

Publisher's note Springer Nature remains neutral with regard to jurisdictional claims in published maps and institutional affiliations.

(C) The Author(s), under exclusive licence to Springer Nature Limited 2020 


\section{Methods}

Mice. All of the mice were housed in specific pathogen-free conditions in an animal facility at the Children's Hospital of Philadelphia. The mice were housed at an ambient temperature of $21 \pm 1{ }^{\circ} \mathrm{C}$ with humidity levels kept at $60 \%$. Lighting in the rooms was on a 12-h cycle such that the lights were on from 06:15 to $18: 15$ and were otherwise kept off. All mouse protocols were approved by the Institutional Animal Care and Use Committee at the Children's Hospital of Philadelphia. The study was compliant with all of the relevant ethical regulations regarding animal research. Mice aged 10-12 weeks were used for the experiments (18-25 g). We strived to use male and female mice in equal proportions. Unless otherwise specified, we used a minimum of three mice per time point per treatment condition in each experiment. Mice were housed and used in accordance with institutional and American Association for Laboratory Animal Care guidelines. $S f t p c^{C r e E R T 2}$ mice $^{70}$ were generously donated by H. A. Chapman at the University of California, San Francisco. Trk $b^{E G F P}$ mice $^{71}$ were generously donated by D. D. Ginty at Harvard University. Trk $B^{L o x P}$ mice $^{72}$ were generated by L. F. Parada, currently at the Memorial Sloan Kettering Cancer Center, and were shared with us by J. O. McNamera at Duke University. All of the other mice were obtained from The Jackson Laboratory: C57BL/6 (stock number 000664), Bdnf ${ }^{\text {Cre }}$ (stock number $030189)^{73}, B d n f^{\text {LoxP }}$ (stock number 004339) ${ }^{74}, H_{o p x^{3 F l a g G F P}}$ (stock number 029271) ${ }^{75}$,

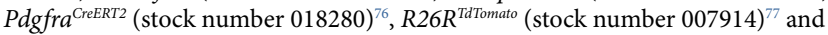
Stat ${ }^{\text {LoxP/LoxP }}$ (stock number 016923) ${ }^{78}$

Acid-induced lung injury. Sedated mice were intubated using a $20 \mathrm{G}$ angiocatheter (BD Biosciences; 381434 ) using a previously described technique ${ }^{79}$. The mice were then placed in the right lateral recumbent position and a polyethylene 10 catheter (BD Biosciences; 427400) was directed into the right main stem bronchus as previously described ${ }^{80}$. Injury was induced by instilling $2 \mu \mathrm{lg}^{-1}$ of osmotically balanced $0.1 \mathrm{~N} \mathrm{HCl}$ into the right lung through the polyethylene 10 catheter. Where indicated, mice received intraperitoneal injections of $10 \mathrm{mg} \mathrm{kg}^{-1} 7,8$-DHF (SigmaAldrich; D5446) in dimethyl sulfoxide (DMSO) and phosphate-buffered saline (PBS) (1:1 ratio) on the day of acid instillation, followed by daily intraperitoneal injections. Mice in the control group received an identical volume of DMSO and PBS at the same time points.

Influenza lung injury. PR8 H1N1 influenza was a generous gift from C. B. Lopez at the University of Pennsylvania. For infection, the virus was diluted in PBS and a dose of $5 \times 10^{-5}$ HAU per mouse was administered via intranasal instillation. Following infection, animals were weighed and monitored three times per week (Monday, Wednesday and Friday) for up to $21 \mathrm{~d}$. Animals that lost $>30 \%$ of their starting weight or were moribund were euthanized humanely. Where indicated, mice received intraperitoneal injections of $10 \mathrm{mg} \mathrm{kg}^{-1}$ 7,8-DHF (Sigma-Aldrich; D5446) or vehicle control on the day of infection and on subsequent Mondays, Wednesdays and Fridays. Mice in the control group received an identical volume of DMSO and PBS at the same time points.

Isolation of murine AT2 and mesenchymal cells. Murine lung tissue was digested into a single-cell suspension, as previously described, using dispase (Corning; 354235), collagenase (Roche; 10103578001) and DNase (Roche; 10104159001). When isolating AT2 cells from $S f t p c^{\text {CreERT2 }}: R 26 R^{\text {TdTomato }}$ mice, the animals received $200 \mathrm{\mu g} \mathrm{gm}^{-1}$ tamoxifen (Sigma-Aldrich; T55648) in corn oil (Sigma-Aldrich; C8267) via gavage $3 \mathrm{~d}$ before being euthanized. Tomato ${ }^{+}$cells were isolated using flow cytometry (MoFlo Astrios with Summit version 6.3.0.16900 software). To isolate AT2 cells from mice, we stained single-cell suspensions of murine lung tissue and gated based on the following criteria: positive for EpCAM-APC (BioLegend; 118213) and negative for CD31-PE (eBioscience; 12-0311-81), CD45-PE (eBioscience; 12-0451-81), podoplanin-PE (eBioscience; 12-5381-80), Sca1-PE (eBioscience; 12-5981-81), CD24-PE (BioLegend; 119307) and DAPI (BioLegend; 422801), as previously described ${ }^{81}$. To isolate mesenchymal cells, we sorted for cells that were positive for CD140a (BioLegend; 135907) and negative for DAPI (BioLegend; 422801). Antibody concentrations are shown in Supplementary Table 2.

Isolation of human AT2 cells. Samples of uninjured, de-identified human lungs were obtained from non-utilized lungs donated for organ transplantation via an established protocol for the Prospective Registry of Outcomes in Patients Electing Lung Transplantation Study approved by the University of Pennsylvania Institutional Review Board with informed consent in accordance with institutional procedures. A $2 \mathrm{~cm} \times 2 \mathrm{~cm}$ piece of distal lung tissue was obtained, pleura and large airways were carefully dissected away, and tissue was processed into a single-cell suspension using the same combination of dispase, collagenase I and DNase used for mouse lungs. A Miltenyi gentleMACS dissociator was used for mincing and incubation for $35 \mathrm{~min}$ at $37^{\circ} \mathrm{C}$. Cells were washed and passed over $70-\mu \mathrm{M}$ and $40-\mu \mathrm{M}$ filters, and red blood cells were lysed with ammonium-chloride-potassium (ACK) lysis buffer. After a single-cell suspension was obtained and cells were sorted using the MACS MultiSort Kit, MACS LS columns and the following antibodies: HT2-280 (mouse immunoglobulin M), a gift from L. Dobbs at the University of California, San Francisco; and anti-mouse immunoglobulin M microbeads (Miltenyi; 130-047-302). The full protocol for digestion and sorting of human lung epithelial cells, and their propagation as alveolar organoids, has been made available via the Nature Protocol Exchange ${ }^{82}$.

Organoid assay. Clonal alveolar organoid assays were performed as described previously with some modifications from the original protocol ${ }^{17,37,81}$. In brief, $5 \times 10^{3}$ AT2 cells were isolated as described above and mixed with $5 \times 10^{4}$ lung PDGFR $\alpha^{+}$isolated cells from adult wild-type mice, as previously described ${ }^{19}$ for mouse or MRC5 cells (ATCC CCL-171, tested negative for mycobacterial contamination, at no greater than passage 7), for human cultures. For the first $2 \mathrm{~d}$ of culture, ROCK inhibitor Y27632 (Sigma-Aldrich; Y0503) was added to the media (SAGM; Lonza; CC-3118). After $2 \mathrm{~d}$ of culture, Y276632 was removed and ligand treatments of organoids were performed using the following reagents at the indicated concentrations: ANA-12 $\left(0.02 \mu \mathrm{g} \mathrm{ml}^{-1}\right.$; Alomone Labs; A-215), recombinant human BDNF $\left(0.05 \mu \mathrm{g} \mathrm{ml}^{-1}\right.$; Alomone Labs; B-250) and recombinant murine FGF7 $\left(0.01 \mu \mathrm{g} \mathrm{ml}^{-1}\right.$; R\&D Systems; 5028-KG). DMSO was used as a control for the ANA-12, whereas $2 \%$ bovine serum albumin was used as a control for the BDNF and FGF7. The media was changed every $48 \mathrm{~h}$ and fresh ligands were included at each media change. After $21 \mathrm{~d}$ (or $28 \mathrm{~d}$ where indicated), organoids were imaged using an Olympus MVX10 microscope or EVOS FL microscope (Life Technologies).

ATAC-seq. Individual ATAC-seq libraries were generated from sorted AT2 cells using the methods outlined above and previously described ${ }^{83}$. Briefly, $5 \times 10^{4}$ cells were sorted into media, washed and lysed to obtain nuclei. Nuclei were exposed to Tn5 transposase (Illumina; FC-121-1030) and fractionated DNA was used for amplification and library preparation. Libraries were then purified and underwent paired-end sequencing (100 base pairs (bp)) using the Illumina HiSeq 4000. After sequencing, the adapters were trimmed with atactk (version 0.1 .5 ; https://atactk. readthedocs.io/en/latest/index.html) and raw reads were aligned against the mouse reference genome $(\mathrm{mm} 9)$ using the Bowtie 1.1.2 aligner with the following flags: --chunkmbs 2000 --sam --best --strata -m1 -X 2000 (ref. ${ }^{84}$ ). SAMtools and Picard tools were used to exclude duplicate reads and reads mapping to ChrM were excluded from further analysis. We used MACS2 (ref. ${ }^{85}$ ) for peak calling with a $q$ cutoff of 0.05 . Downstream analysis and visualization was done using HOMER ${ }^{40}$ and deepTools2 (ref. ${ }^{86}$ ).

scRNA-seq. Murine AT2 cells were isolated using the above protocols. Single cells were captured using the $10 \times$ Genomics microfluidic platform. RNA was isolated and libraries prepared using $10 \times$ Genomics reagents according to the manufacturer's protocol. Sequencing was performed on an Illumina HiSeq 2500 using asymmetrical reads, as suggested by $10 \times$ Genomics. We downloaded data on the single-cell RNA expression of pulmonary mesenchymal cells from a previous publication ${ }^{46}$ whose data were deposited in the Gene Expression Omnibus (GSE99714). Fastq files were assessed for quality control using the FastQC program (https://www.bioinformatics.babraham.ac.uk/projects/fastqc). Fastq files were aligned against the mouse reference genome $(\mathrm{mm} 9)$ using the Cell Ranger environment (10× Genomics). t-SNE clustering and differential expression analyses were done using Seurat ${ }^{87}$.

Pathways analysis. The differential genes between clusters were identified using the $\mathrm{R}$ package Cell Ranger $\mathrm{R}$ Kit. These lists were then analysed using ingenuity pathway analysis (Qiagen; https://www.qiagenbioinformatics.com/products/ ingenuity-pathway-analysis). The final heatmap was visualized using the gplots R package.

Histology. At the time of tissue harvest, mice were euthanized by $\mathrm{CO}_{2}$ inhalation. The chest cavity was exposed and the lungs cleared of blood by perfusion with cold $\mathrm{PBS}$ via the right ventricle. Lungs were inflated with $4 \%$ paraformaldehyde under a constant pressure of $30 \mathrm{~cm}$ water and allowed to fix for $24 \mathrm{~h}$. Tissue was then dehydrated, paraffin embedded and sectioned. Haematoxylin and eosin staining was performed to examine the morphology, and to score regions based on the severity of injury using the ATS/European Respiratory Society (ERS) criteria $^{88}$. Immunohistochemistry was used to detect protein expression using the following antibodies on paraffin sections: DC-LAMP (Novus Biologicals; DDX0191P-100), GFP (Molecular Probes; A1122), Ki67 (Abcam; ab16667), KRT5 (BioLegend; 905501 and 905903), PDGFR $\alpha$ (Cell Signaling Technology; 3177), PECAM-1 (Dianova; DIA-310), Podoplanin (University of Iowa Developmental Studies Hybridoma Bank clone 8.1.1), pSTAT3 (Cell Signaling Technology; 9145), proSFTPC (MilliporeSigma; AB3786) and RFP (Rockland Immunochemicals; 600-401-379). Antibody concentrations are shown in Supplementary Table 2.

Image acquisition. Confocal immunofluorescent images were obtained using the Leica TCS SP8 scanning confocal microscope using the Leica LAS X software (version 3.0.13). Images of haematoxylin and eosin-stained sections were obtained using the Aperio Image Scope (version 12.4).

Quantification of pSTAT3 in AT2 cells. Histological specimens were obtained and stained as outlined above. Random confocal images were randomly obtained by 
an operator who was unaware of the experimental conditions or hypothesis. The digital sections were then reviewed by a blinded investigator who identified cells that were co-positive for proSFTPC and pSTAT3, as well as cells that were positive for proSFTPC but not pSTAT3. The blinded investigator also identified several areas where they did not feel confident making a determination. Reasons for uncertainly included autofluorescence of red blood cells, unclear staining patterns or adjacent nuclei being too close to each other to accurately distinguish between adjacent cells.

Quantitative PCR. RNA was isolated from sorted mesenchymal cells or AT2 cells using the RNeasy Mini Kit (Qiagen; 74104) and reverse transcribed using the High-Capacity RNA-to-cDNA Kit (Applied Biosystems; 4387406). Quantitative PCR was performed using primers specific for Bdnf, Fgf7, Hprt 1 and TrkB with the Power SYBR Green PCR Master Mix (Applied Biosystems; 4367659). Quantitative PCR was performed using the ViiA 7 Real-Time PCR System (Applied Biosystems). Primer sequences are shown in Supplementary Table 3 .

PCR. RNA was isolated from sorted AT2 cells using the RNeasy Mini Kit (Qiagen; 74104) and reverse transcribed using the High-Capacity RNA-to-cDNA Kit (Applied Biosystems; 4387406). PCR was performed with the primers specific for STAT3. The expected product was $807 \mathrm{bp}$ without Cre-mediated recombination and $519 \mathrm{bp}$ after Cre-mediated recombination. Primer sequences are shown in Supplementary Table 4

Enzyme-linked immunosorbent assays (ELISAs) and protein assays. BAL samples were obtained and cell counts were analysed as previously described ${ }^{89}$ Levels of IL-1 $\beta$ were measured using an ELISA kit (eBioscience; 501129749). Levels of IL-6 were measured using an ELISA kit (eBioscience; 88-7064-88). Protein assays were performed using the Microplate BCA Protein Assay Kit (Thermo Fisher Scientific; 23252). All plates were read on the SpectraMax 250 spectrophotometer (Molecular Devices).

ATS lung injury scoring. A blinded reviewer examined random haematoxylin and eosin sections from three or four mice per time point per condition and scored each mouse according to published guidelines ${ }^{88}$.

Calculation of alveolar septal thickness and mean linear intercepts. We used the MATLAB 2018a (RRID: SCR_001622) image processing and statistical toolboxes to perform high-throughput alveolar wall thickness analysis and mean length intercept analysis of haematoxylin and eosin-stained sections taken under a brightfield microscope with a $20 \times$ objective. We obtained ten random images from each influenza-infected mouse and five random images from mice that underwent acid-induced lung injury. We obtained fewer images from the acid-injured mice because the acid injury was unilateral. The program performed binarization using automated thresholding based on Otsu's method. It performed erosion, then dilation to fill the holes in the alveolar walls, and inverted the image such that the alveolar spaces were black and the walls were white. Subsequently, the program used the Laplacian operator to extract the boundaries of the alveolar spaces, and using the bwboundaries function, detected alveolar spaces as distinct objects. The object detection enabled computation of the minimum distance between each alveolar space in an iterative manner where distances between each alveolar space and the neighbouring spaces were compared and minimum distances corresponding to the narrowest segments were extracted. About 100-200 measurements per field were taken and the average value representing the mean interseptal wall thickness was calculated. For the mean linear intercept, the dimensions of the image were extracted and based on the vertical dimension, and an equally spaced line grid consisting of 20 lines was created. The number of times the lines intersected with the walls and the length of the intercepts for each of the lines based on dimensions of the image were calculated. The mean length of the intercept for each mouse is reported.

Statistics and reproducibility. Statistical analysis was performed in Prism for Mac (version 8.4.2) and R. Pvalues were obtained using two-tailed Student's $t$-tests for comparison of two datasets or by analysis and variance (ANOVA) where appropriate. Statistical analysis of transcription factor binding motifs in Fig. $1 \mathrm{~g}$ was done using the findMotifGenome.pl script in the HOMER software package, with adjustment for multiple comparisons. Odds ratios for the distribution of ATAC regions near genes were calculated using Fisher's exact test and contingency table analysis. Statistical data were considered significant if $P<0.05$. Centre values of all plots represent means and error bars represent s.e.m. The results were reproducible and conducted with established internal controls. When feasible, experiments were repeated three or more times and yielded similar results. We have indicated the $n$ values used for each analysis in the figure captions. Cell cultures were routinely screened for Mycoplasma.

Reporting Summary. Further information on research design is available in the Nature Research Reporting Summary linked to this article.

\section{Data availability}

ATAC-seq and scRNA-seq data that support the findings of this study have been deposited in the Gene Expression Omnibus under accession code GSE132535. All other data supporting the findings of this study are available from the corresponding author on reasonable request. Source data are provided with this paper.

\section{References}

70. Chapman, H. A. et al. Integrin $\alpha 6 \beta 4$ identifies an adult distal lung epithelial population with regenerative potential in mice. J. Clin. Invest. 121, 2855-2862 (2011)

71. Li, L. et al. The functional organization of cutaneous low-threshold mechanosensory neurons. Cell 147, 1615-1627 (2011).

72. Luikart, B. W., Nef, S., Shipman, T. \& Parada, L. F. In vivo role of truncated TrkB receptors during sensory ganglion neurogenesis. Neuroscience 117, 847-858 (2003).

73. Tan, C. L. et al. Warm-sensitive neurons that control body temperature. Cell 167, 47-59.e15 (2016)

74. Rios, M. et al. Conditional deletion of brain-derived neurotrophic factor in the postnatal brain leads to obesity and hyperactivity. Mol. Endocrinol. 15, 1748-1757 (2001)

75. Takeda, N. et al. Hopx expression defines a subset of multipotent hair follicle stem cells and a progenitor population primed to give rise to $\mathrm{K}^{+}$niche cells. Development 140, 1655-1664 (2013).

76. Chen, Y. et al. Thermal stress induces glycolytic beige fat formation via a myogenic state. Nature 565, 180-185 (2019).

77. Madisen, L. et al. A robust and high-throughput Cre reporting and characterization system for the whole mouse brain. Nat. Neurosci. 13, 133-140 (2010).

78. Moh, A. et al. Role of STAT3 in liver regeneration: survival, DNA synthesis, inflammatory reaction and liver mass recovery. Lab. Invest. 87, 1018-1028 (2007).

79. Das, S., MacDonald, K., Chang, H. Y. \& Mitzner, W. A simple method of mouse lung intubation. J. Vis. Exp. 73, e50318 (2013).

80. Paris, A. J. et al. Using selective lung injury to improve murine models of spatially heterogeneous lung diseases. PLoS ONE 14, e0202456 (2019).

81. Alder, J. K. et al. Telomere dysfunction causes alveolar stem cell failure. Proc. Natl Acad. Sci. USA 112, 5099-5104 (2015).

82. Zacharias, W. \& Morrisey, E. Isolation and culture of human alveolar epithelial progenitor cells. Nat. Protoc. Exchange http://dx.doi.org/10.1038/ protex.2018.015 (2018).

83. Buenrostro, J. D., Wu, B., Chang, H. Y. \& Greenleaf, W. J. ATAC-seq: a method for assaying chromatin accessibility genome-wide. Curr. Protoc. Mol. Biol. 109, 21.29.1-29.29.9 (2015).

84. Langmead, B., Trapnell, C., Pop, M. \& Salzberg, S. L. Ultrafast and memoryefficient alignment of short DNA sequences to the human genome. Genome Biol. 10, R25 (2009).

85. Zhang, Y. et al. Model-based analysis of ChIP-Seq (MACS). Genome Biol. 9, R137 (2008)

86. Ramírez, F. et al. deepTools2: a next generation web server for deep-sequencing data analysis. Nucleic Acids Res. 44, W160-W165 (2016).

87. Butler, A., Hoffman, P., Smibert, P., Papalexi, E. \& Satija, R. Integrating single-cell transcriptomic data across different conditions, technologies, and species. Nat. Biotechnol. 36, 411-420 (2018).

88. Matute-Bello, G. et al. An official American Thoracic Society workshop report: features and measurements of experimental acute lung injury in animals. Am. J. Respir. Cell Mol. Biol. 44, 725-738 (2011).

89. Nick, J. A. et al. Selective suppression of neutrophil accumulation in ongoing pulmonary inflammation by systemic inhibition of p38 mitogen-activated protein kinase. J. Immunol. 169, 5260-5269 (2002).

\section{Acknowledgements}

We acknowledge S. M. Albelda, J. S. Brenner and A. E. Vaughan for help with preparing this manuscript, as well as the Pathology, Flow Cytometry, Nucleic Acid and PCR cores and the Center for Applied Genomics at the Children's Hospital of Philadelphia Research Institute. This work was supported by the Parker B. Fellowship Program (A.J.P.) and multiple grants from the National Institutes of Health (K08 HL136698 (A.J.P.), R01 AI121321 (M.D.W.), R01 AI099479 (G.S.W.) and R01 DK114054 (G.S.W.)). We also thank the patients and families who donated lung tissue to support this research.

\section{Author contributions}

A.J.P. conceived of and co-performed the ATAC-seq experiment, performed all of the acid-induced lung injury experiments, analysed the histological data and co-wrote the manuscript. K.E.H. analysed the ATAC-seq and scRNA-seq data. J.H.O. assisted with the data analysis and co-wrote the manuscript. D.C.A. co-performed the ATAC-seq experiment. S.A.T. performed the influenza injury experiments. J.A.Z. performed the scRNA-seq of mesenchymal cells and assisted with the murine alveolar organoid 
experiments. W.J.Z. assisted with the murine and human alveolar organoid experiments. J.B.K. performed the survival analysis and assisted with isolating the human AT2 cells. M.C.B. identified the human histological samples. M.C.B. and M.M.K. isolated the human AT2 cells. A.R.S. assisted with performing the murine and human organoid experiments. S.J., A.S. and D.B.F. analysed the mean linear intercept and alveolar wall thickness of the murine lung specimens. N.D. and P.W. bred the mice, genotyped the mice, administered the tamoxifen and isolated the murine AT2 cells and fibroblasts. P.W. cultured the MRC5 cells and set up the murine and human organoid experiments. E.C. procured the human lung tissue. G.S.W. blindly scored the histological specimens. L.C.E., M.F.B. and E.E.M. assisted with the experimental design and with preparing the manuscript. M.D.W. and G.S.W. designed, conceived of and supervised the experiments and co-wrote the manuscript. All authors reviewed the manuscript.

\section{Competing interests}

The authors have applied for a provisional patent in the United States related to this work. A.J.P. and G.S.W. are listed as co-inventors on this application.

\section{Additional information}

Extended data is available for this paper at https://doi.org/10.1038/s41556-020-0569-x. Supplementary information is available for this paper at https://doi.org/10.1038/ s41556-020-0569-x.

Correspondence and requests for materials should be addressed to G.S.W.

Reprints and permissions information is available at www.nature.com/reprints. 
Unstained
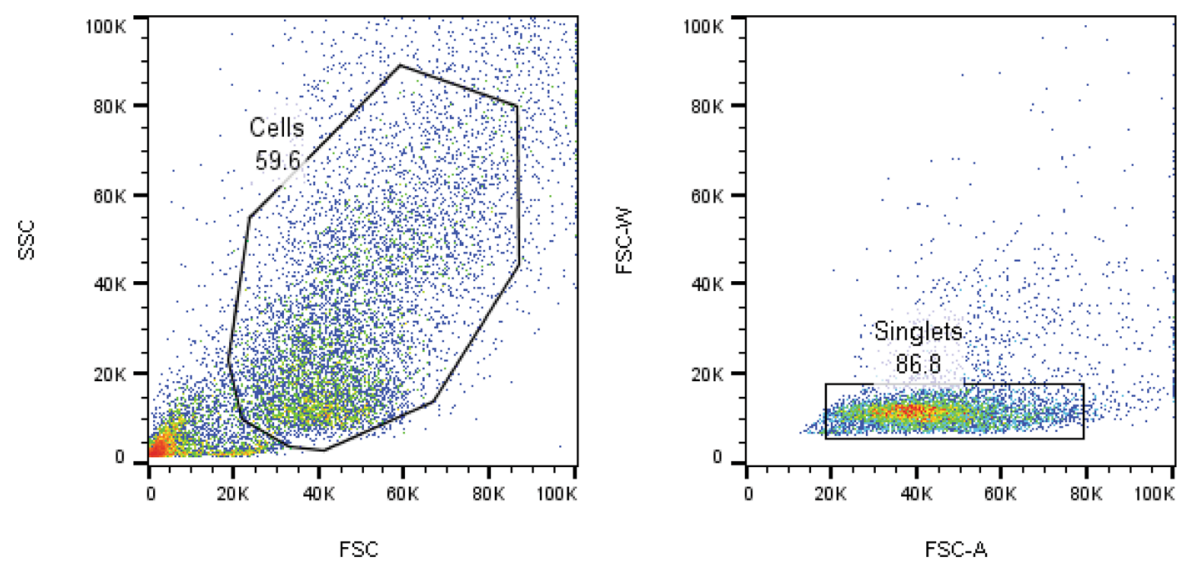

Stained
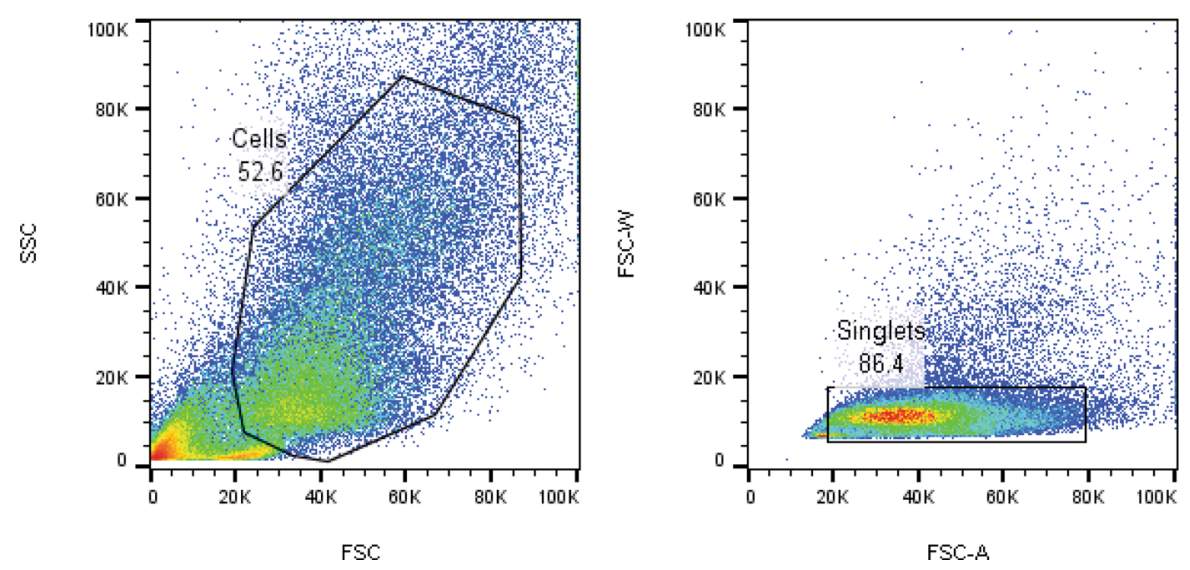

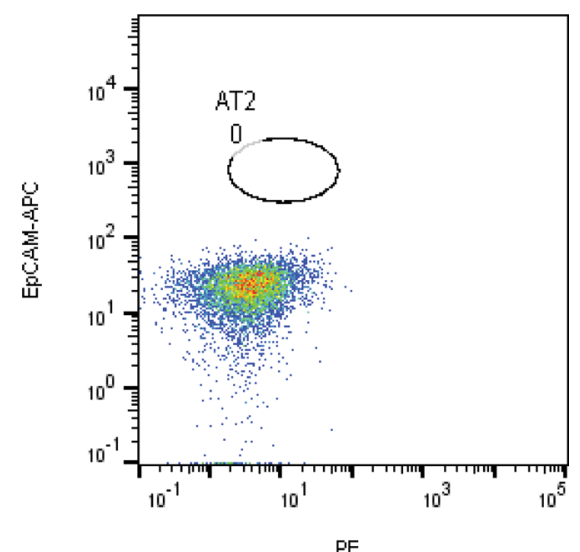

PE

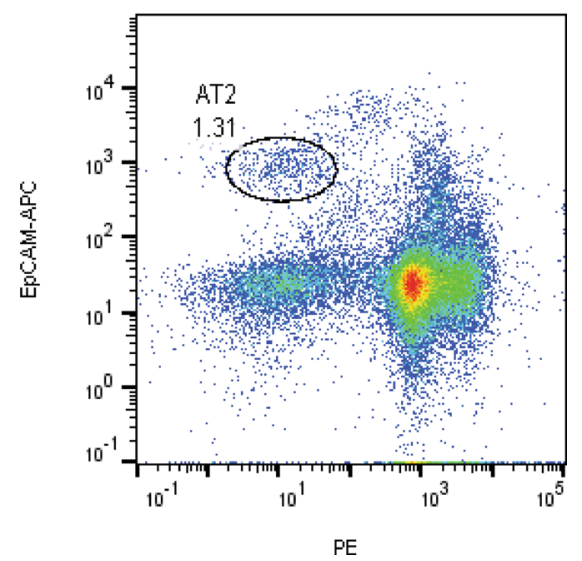

Extended Data Fig. 1 | Sorting Strategies for Isolating AT2 cells. Lungs from C57BL/6 mice were digested into a single-cell suspension. We collected EpCAM positive cells (APC) that were PDPN, CD45, CD34, CD31 and SCA1 negative (all PE). 
Influenza

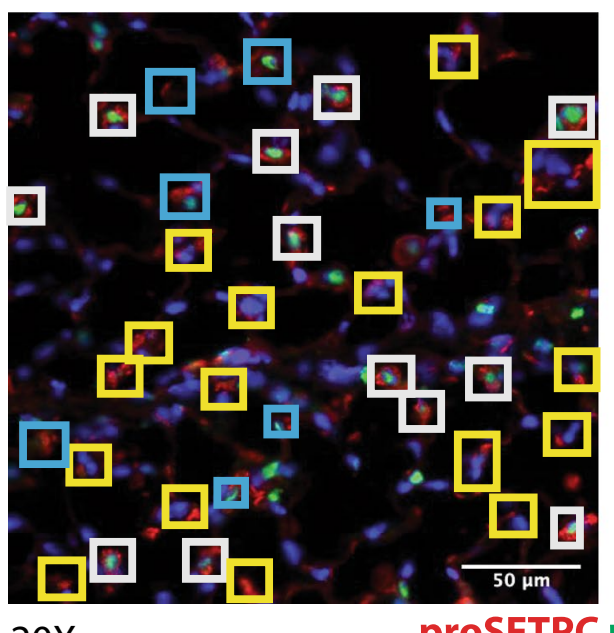

\section{Acid}

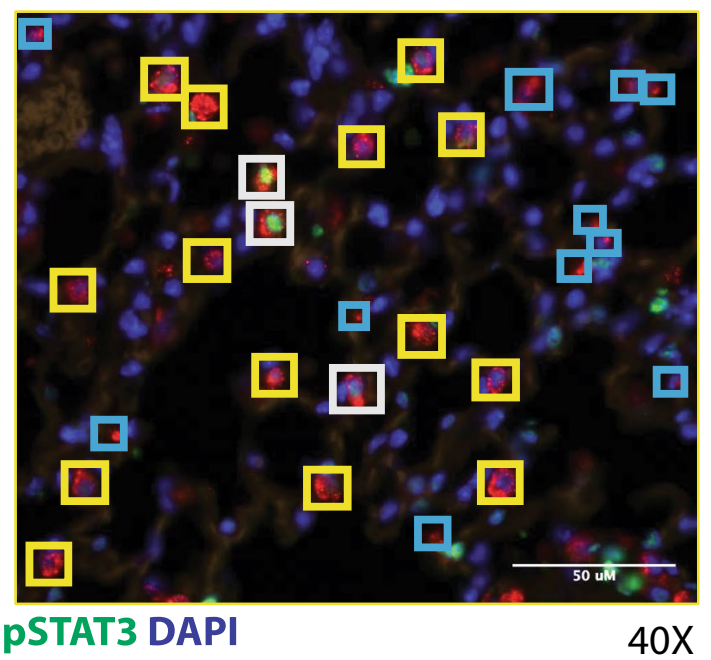

Extended Data Fig. 2 | STAT3 is activated following sterile and infectious lung injuries. Cells staining positive for proSFTPC + and pSTAT3+ are highlighted with a white box while proSFTPC + pSTAT3- cells are highlighted with a yellow box. Areas that were felt to be indeterminate for cell type or pSTAT3 status are highlighted in blue. Scale bars, $50 \mu \mathrm{m}$. 
A

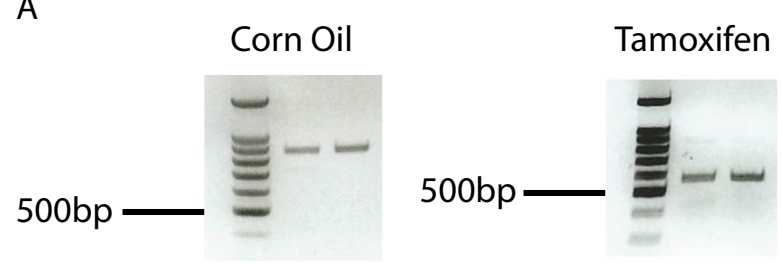

B

Sftpc CreERT2-Rosa26TdTomato

Sftpc CreERT2-Rosa26TdTomato:Stat3 ${ }^{\text {LoxP/LoxP }}$
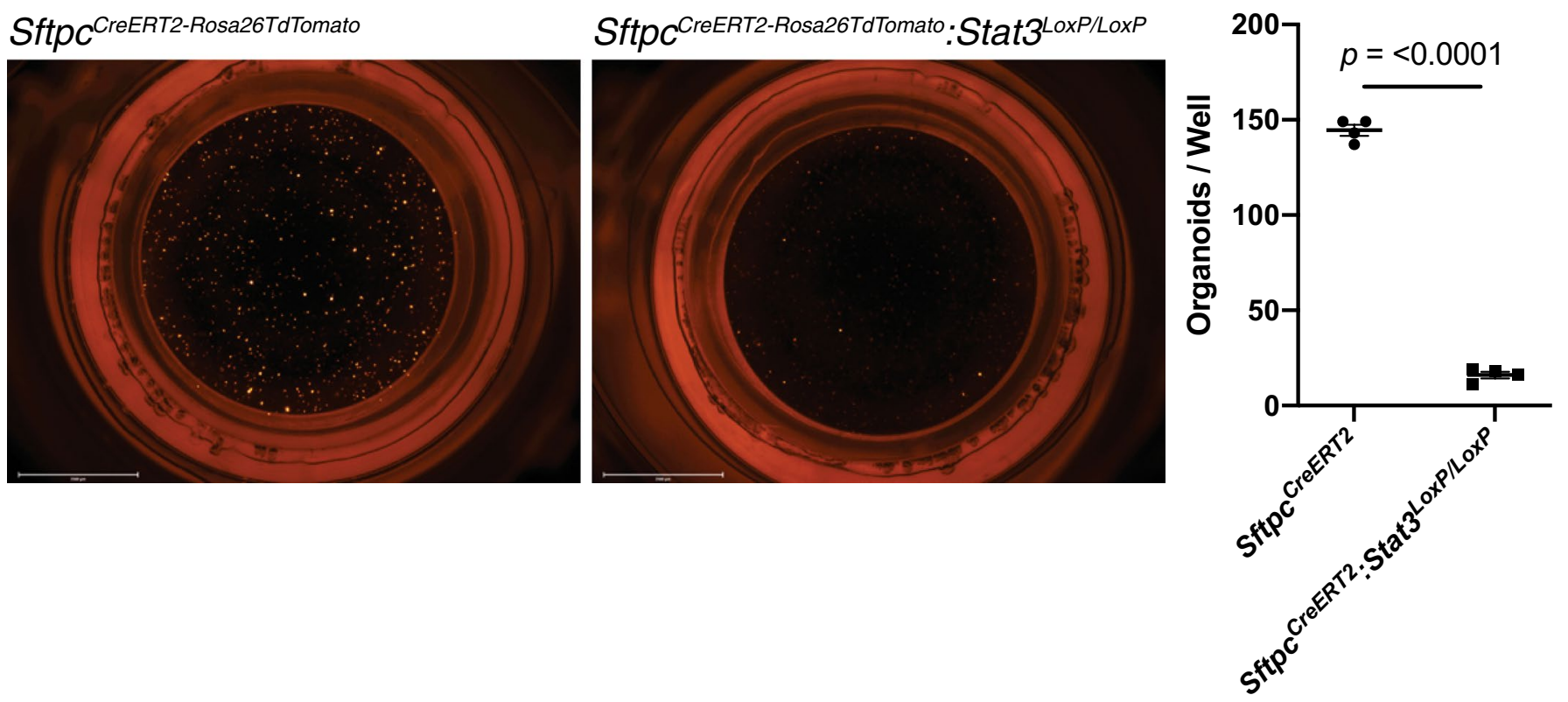

Extended Data Fig. 3 | Loss of Stat3 diminished alveolar organoid formation. a, PCR shows the expected 802bp product when corn oil is administered to Sftpc CreRRT2:Stat3 ${ }^{\text {LoxP/LOXP }}$ mice and the recombined 514bp product when tamoxifen is administered to Sftpc CreeRT2:Stat3 ${ }^{\text {LoxP/LoxP }}$ mice. The gels in this figure represent $\mathrm{n}=2$ mice per condition (corn oil and tamoxifen). This experiment was repeated 4 times with similar results. $\mathbf{b}$, AT2 cells from tamoxifen treated Sftpc CreERT2-Rosa26tdTomato and Sftpc CreERT2-Rosa26tdTomato:Stat ${ }^{\text {LoxP/LoxP }}$ mice were isolated and co-cultured with PDGFR $\alpha$ mesenchymal cells in transwells containing Matrigel for four weeks ( $n=4$ wells per condition). Organoid forming efficiency was significantly decreased in the absence of STAT3 signaling in AT2 cells $\left(p=2.2 \times 10^{-8}\right)$. Data is shown as the mean $+/-$ SEM. Statistical significance was determined using a two-tailed Student's t-test. Scale bars, $2.5 \mathrm{~mm}$. 

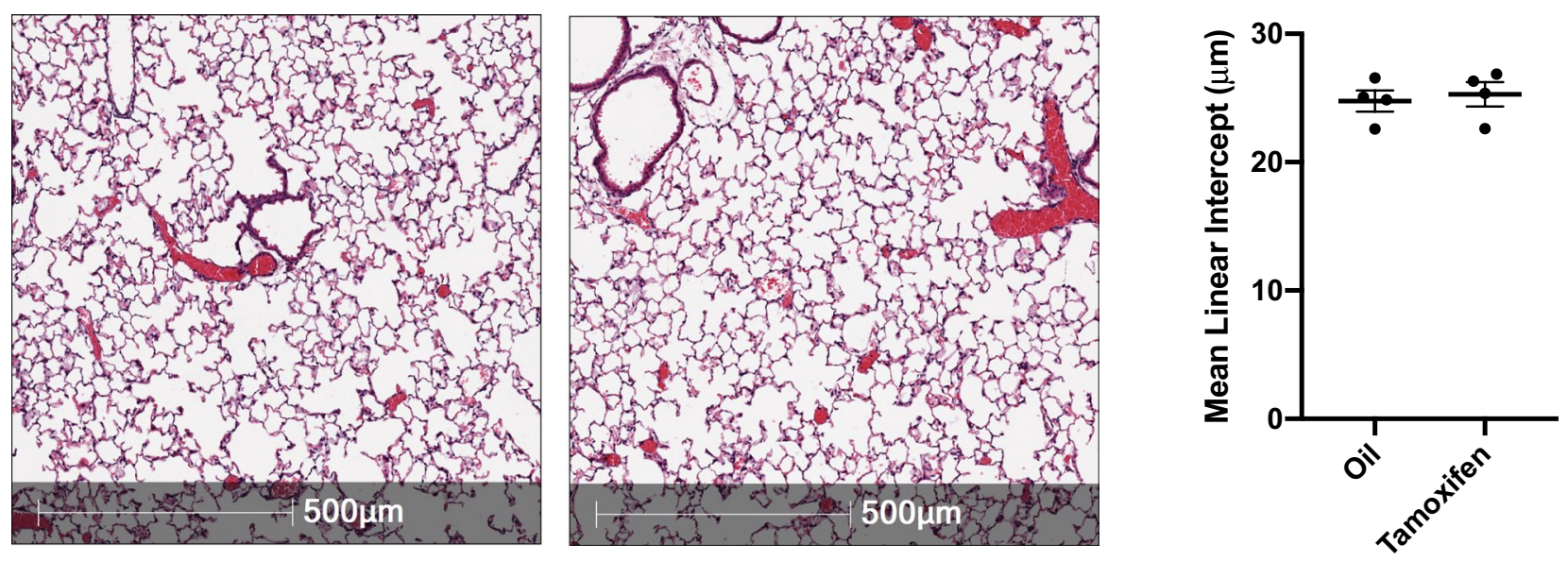

Extended Data Fig. 4 | Loss of Stat3 in AT2 cells does not alter distal lung morphology. Sftpc CreeRT2:Stat3 ${ }^{\text {LoxP/LoxP }}$ mice were given tamoxifen or vehicle and euthanized three months later. Representative H\&E stained histological specimens are shown. The Mean linear intercept was unchanged by the loss of Stat 3 in these unchallenged mice ( $n=4$ mice per group). Data is shown as the mean $+/-$ SEM. Statistical significance was determined with a two-tailed Student's t-test. Scale bars, $500 \mu \mathrm{m}$. 

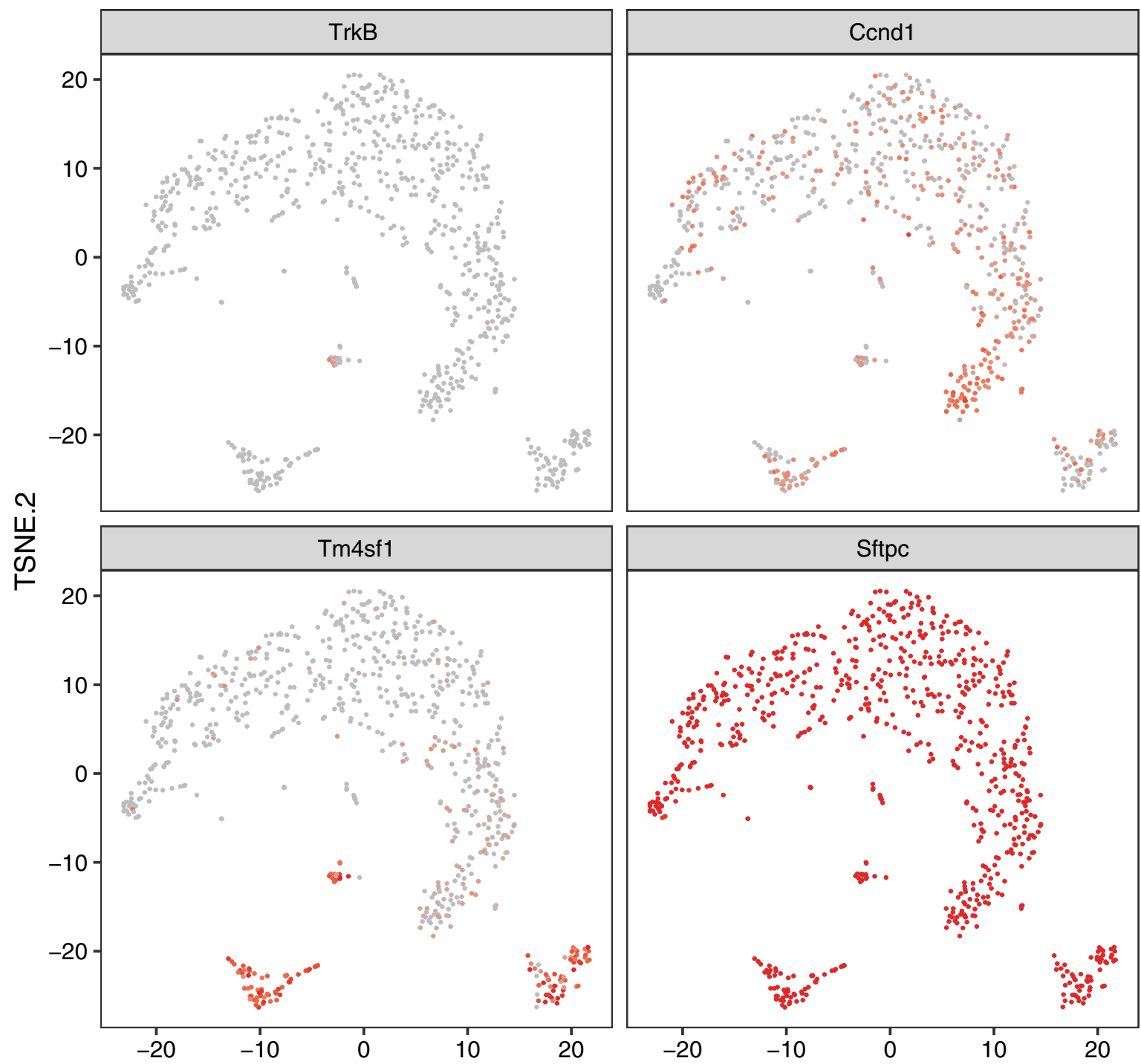

val

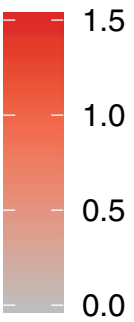

TSNE.1

Extended Data Fig. 5 | Single-cell expression of TrkB, Ccnd1, Tm4sf1 and Sftpc in isolated AT2 cells. Plots show relative expression of TrkB, Ccnd1, Tm4sf1 and Sftpc in AT2 cells isolated isolated from uninjured mice and mice $24 \mathrm{~h}$ after acid-induced lung injury. 

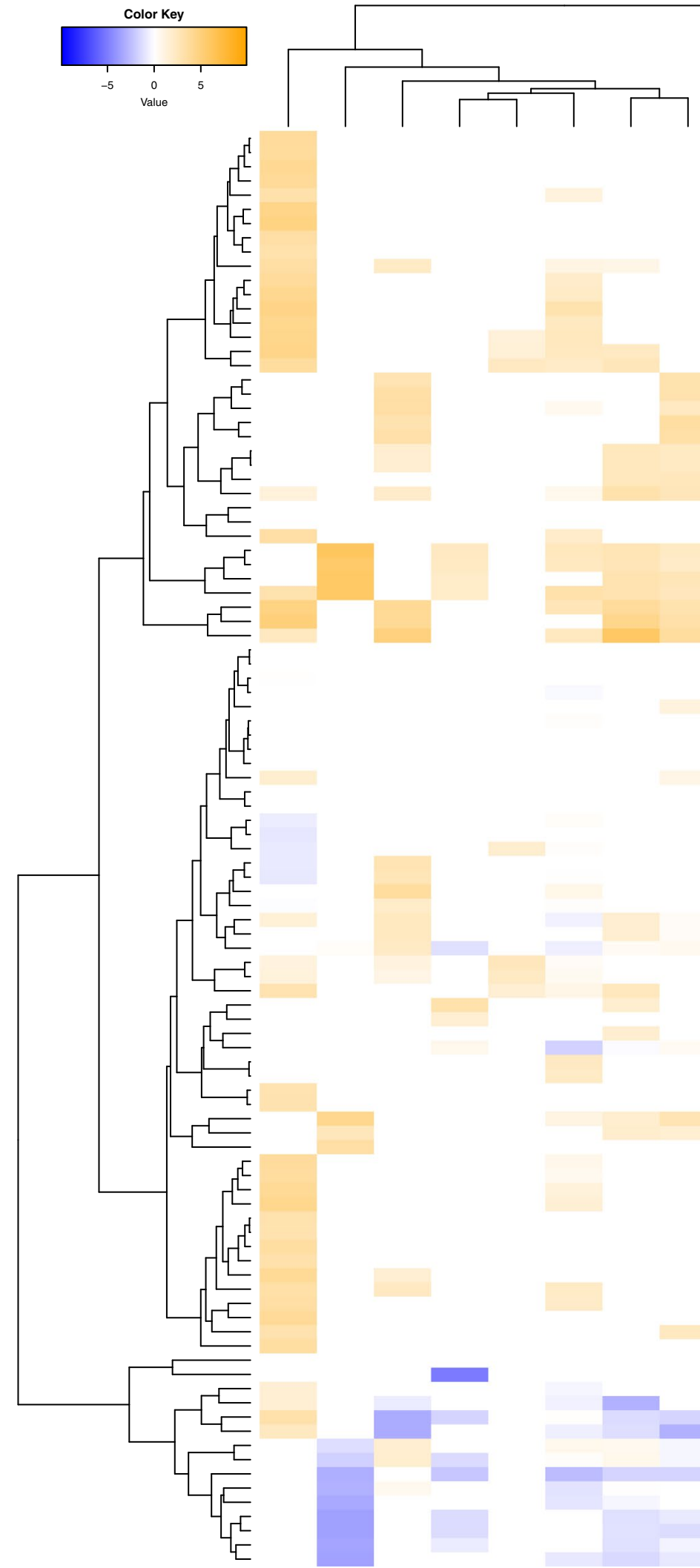

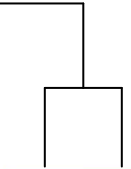

Cell movement of mononuclear leukocytes Migration of mononuclear leukocytes Lymphocyte migration

Cell movement of lymphocytes Leukopoiesis

Binding of leukocytes

Binding of blood cells

Recruitment of leukocytes

Inflammatory respontes

Cell movement of phagocytes

Cell movement of myeloid cells

Adhesion of blood cells

Cell movement of leukocytes

Adhesion of immune cells

Leukocyte migration

Activation of cells

Invasion of cells

Cell proliferation of tumor cell lines

Cell movement of tumor cell lines

Migration of tumor cell lines

Angiogenesis

Development of vasculature

Vasculogenesis

Quantity of cells

Cell movement of blood cells

Microtubule dynamics

Formation of cellular protrusions

Organization of cytoplasm

Organization of cytoskeleton

Migration of cells

Cell movement

Cell survival

Migration of endothelial cells

nals

Quantity of mononuclear leukocytes

Quantity of lymphatic system cells

Interaction of tumor cell lines

Adhesion of tumor cell lines

Transmigration of cells

Cell spreading

Ginding of tumor cell lines

Binding of tumor cell lines
Migration of lymphatic system cells

Migration of lymphatic system cells

Proliferation of immune cells

Proliferation of mononuclear leukocytes

Proliferation of lymphocytes

Proliferation of blood cells

Proliferation of lymphatic system cells

Growth of tumor

Quantity of lymphocyte

Advanced malignant tumo

Quantity of leukocytes

Quantity of blood cells

Cellular homeostasis

Proliferation of neuronal cells

Sprouting

Cavanced stage tumor

Fibrogenesis

Formation of filaments

Homing of leukocytes

Chemotaxis of leukocytes

Development of body trunk

Development of body axis

Formation of cilia

Cellular infiltration by leukocytes

Celuar intilo by leukocytes

Cell movement of neutrophils

Recruitment of neutrophils

Chemotaxis of phagocytes

Accumulation of blood cells

Binding of endothelial cells

Cellular infiltration

Activation of myeloid cells

Activation of leukocytes

Growth of epithelial tissue

Recruitment of phagocytes

Bleeding

Perinatal death

Inflammation of absolute anatomical region

Inflammation of organ

Necrosis

Digestive system cance

Digestive system cance

Devilve organ tumor

Adenocarcinoma

Development of digestive organ tumor

Incidence of tumo

Tumorigenesis of epithelial neoplasm

Devopment of carcinoma

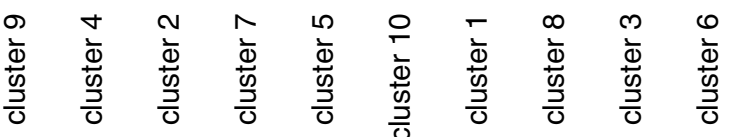

Extended Data Fig. 6 | Pathways analysis of isolated AT2 cells reveals multiple subsets of cells with multiple functions. Pathways analysis of differentially expressed genes in the clusters shown in Fig. 4b. 


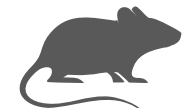

IT Acid

\section{Bdnf Cre-R26RTdTomato}

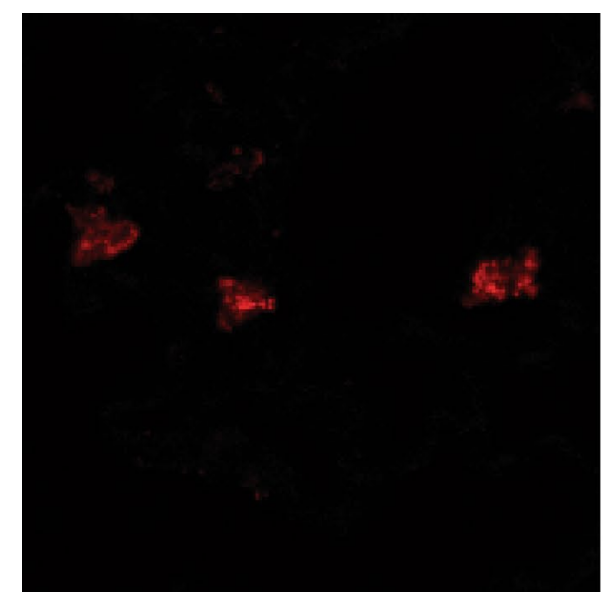

Tomato
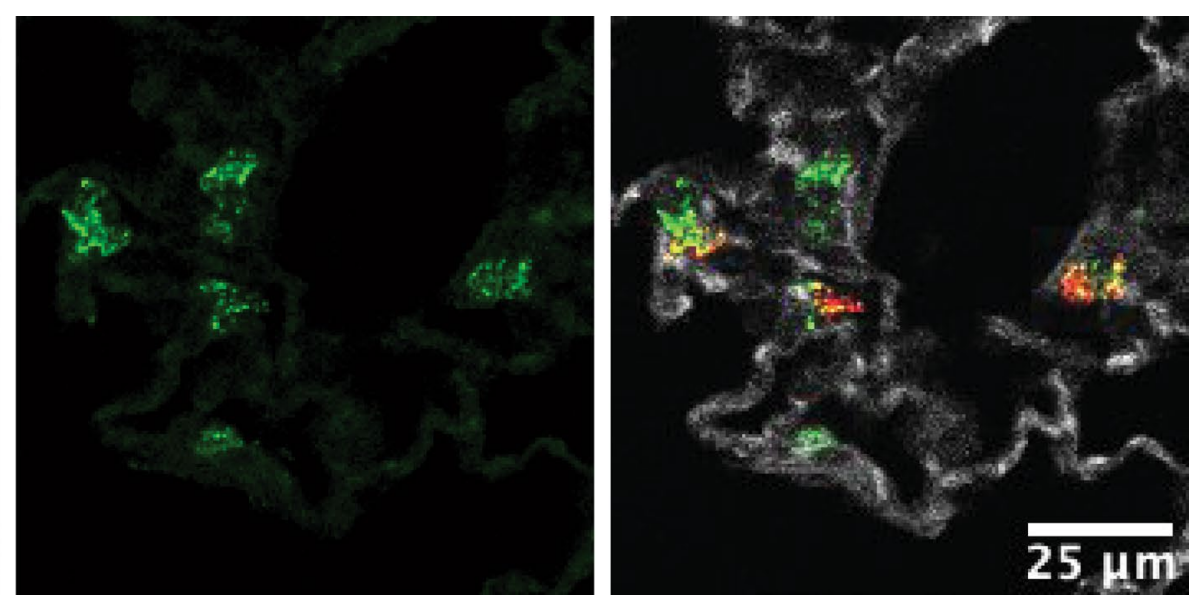

proSftpc
Lungs fixed and stained after $24 \mathrm{~h}$

Extended Data Fig. 7 | AT2 cells express Bdnf after acid-induced lung injury. Bdnfre-Rosa26tdTomato were subjected to acid-induced lung injury ( $\mathrm{n}=4$ mice per group). One day after injury the mice were euthanized, and the lungs were probed for Tomato (red) and proSFTPC (green). Autofluorescence delineates tissue structure. Scale bar, $25 \mu \mathrm{m}$. 
A A
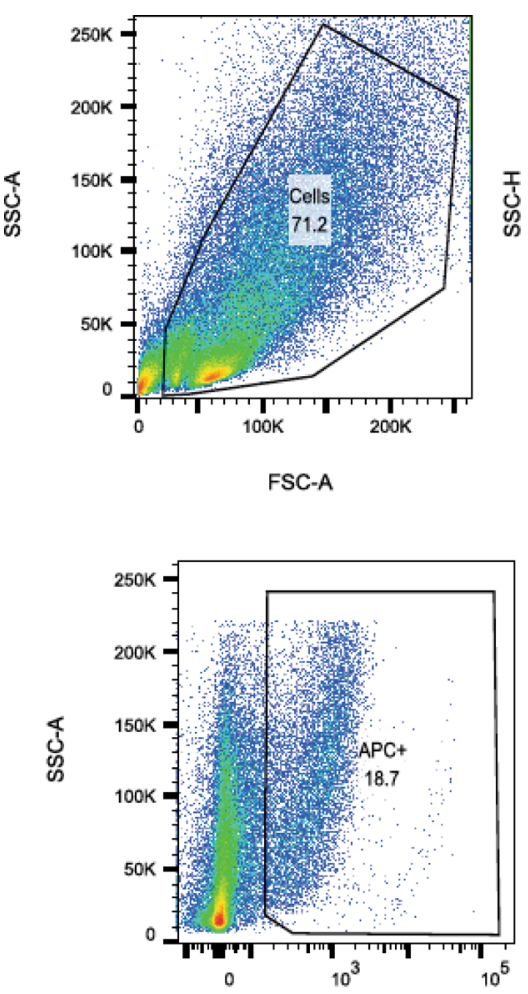

B

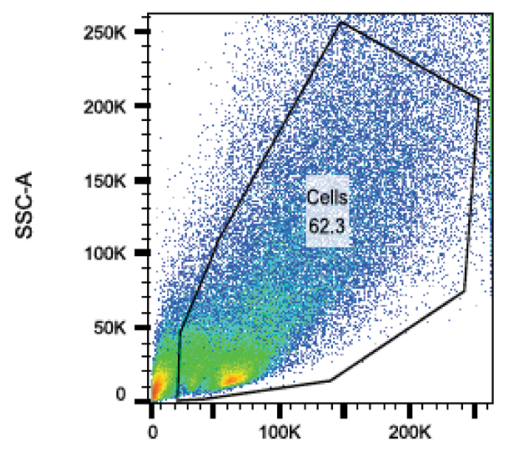

FSC-A

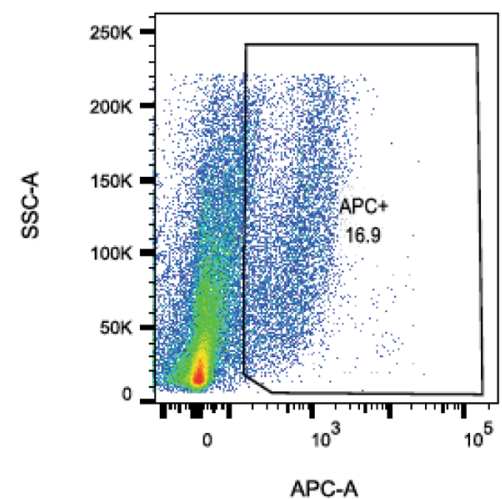

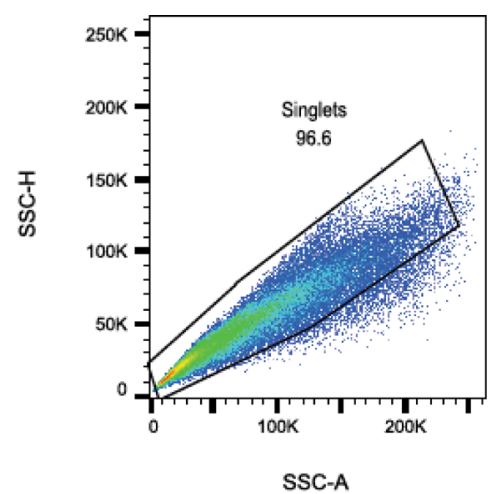
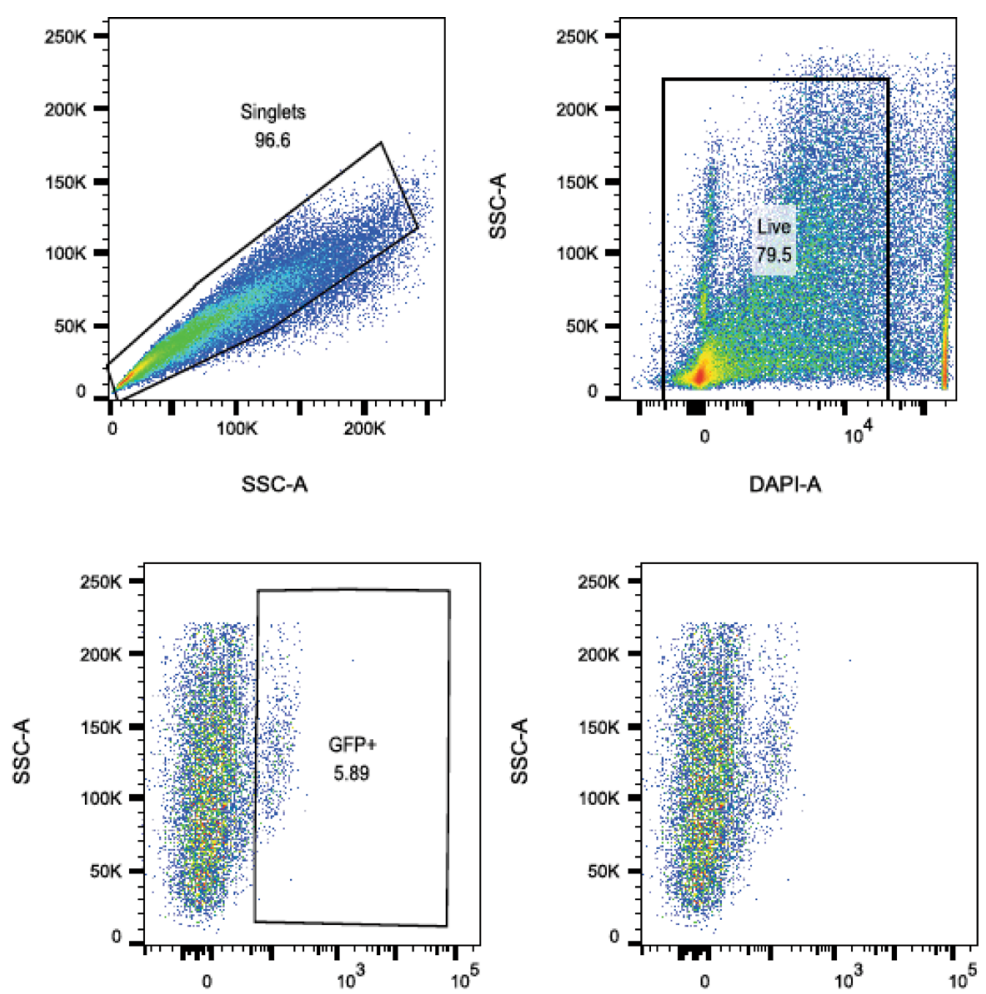

FITC-A
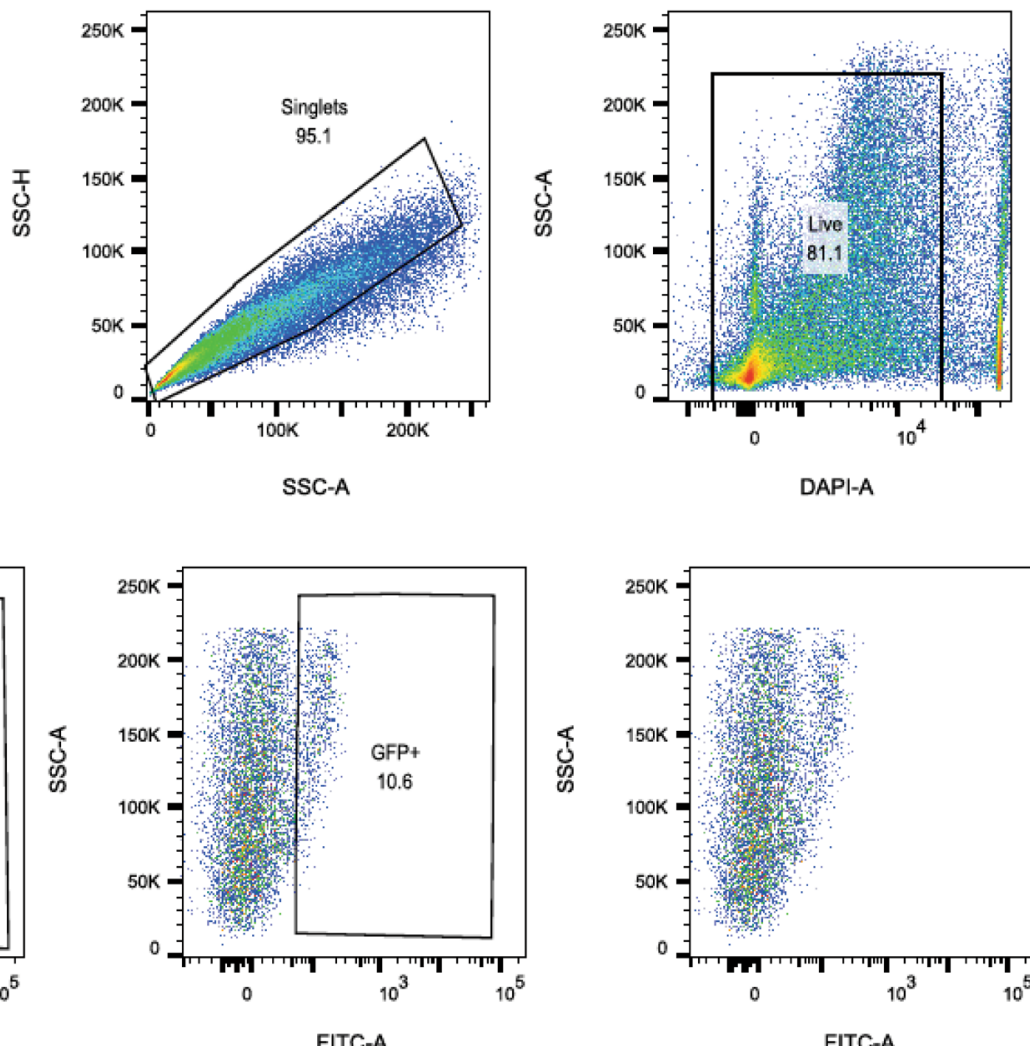

Extended Data Fig. 8 | Loss of TrkB in mesenchymal cells worsens outcomes following sterile and infectious lung injuries. Lungs from Pdgfra ${ }^{\mathrm{EGF}}$ mice were digested into a single-cell suspension either before or $24 \mathrm{~h}$ after acid-induced lung injury ( $\mathrm{n}=4 / \mathrm{group}$ ). We first gated on PDGFR $\alpha+$ and then quantified the percent of GFP+ cells within that population. 
A PdgfraCreERT2:TrkB LoxP/LoxP

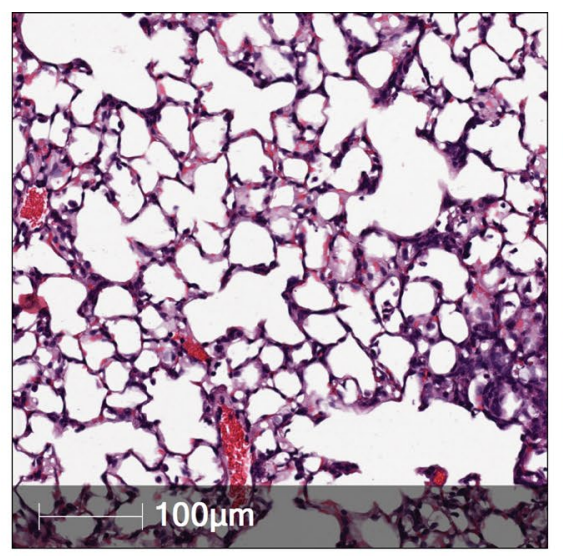

B

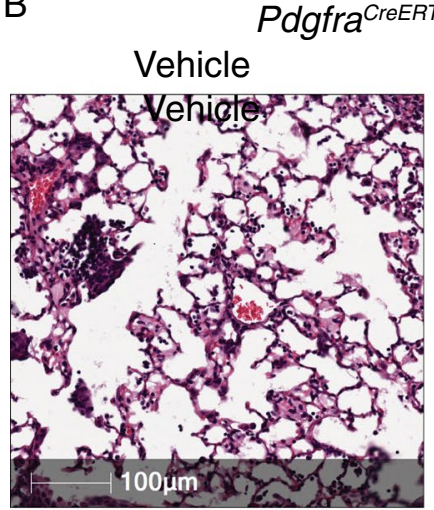

C Pdgfra CreERT2:TrkB LoxP/LoxP

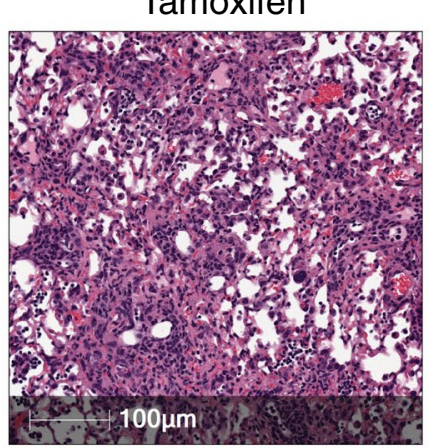

$21 \mathrm{DPI}$

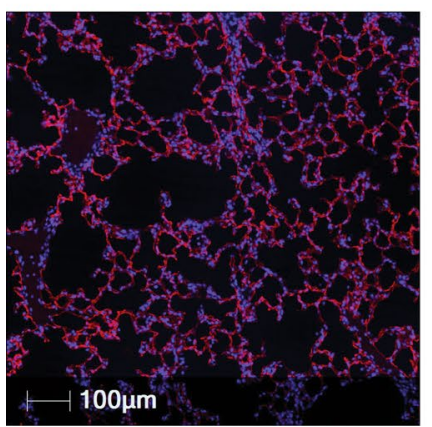

PDPN

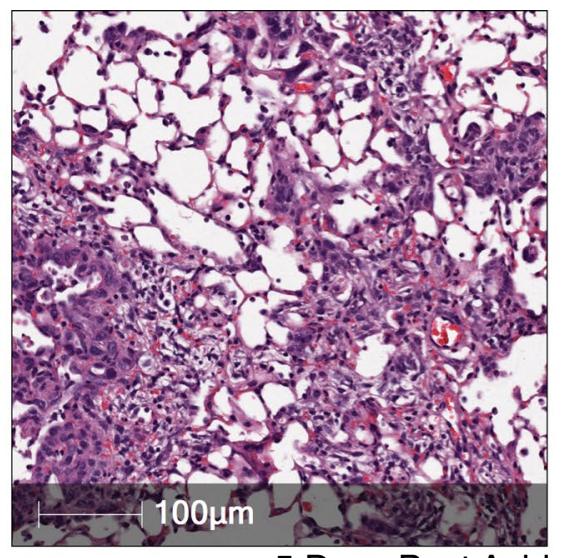

5 Days Post Acid

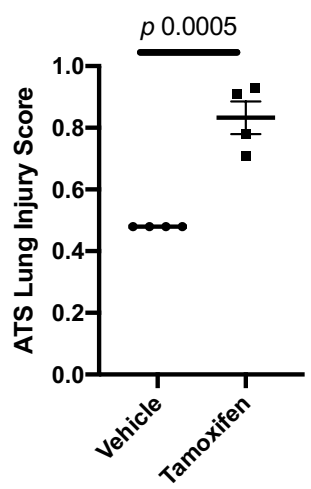

D $\begin{array}{ll}\text { PdgfraCreERT2:TrkB } & \text { LoxP/LOP } \\ \text { Vehicle } & \text { Tamoxifen }\end{array}$

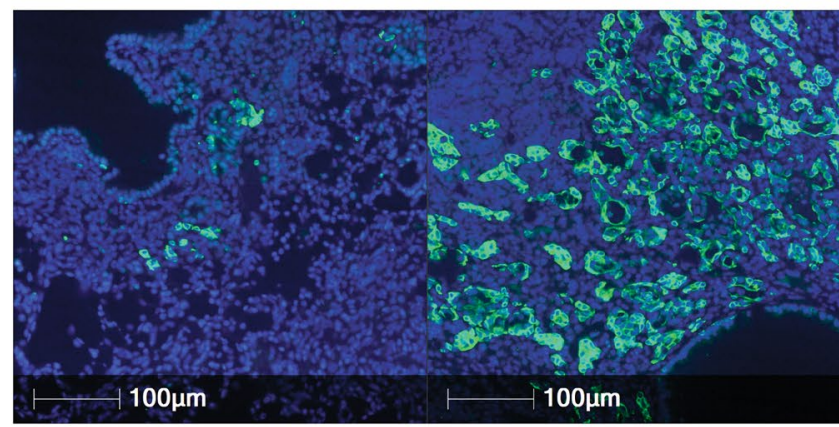

KRT5

$21 \mathrm{DPI}$

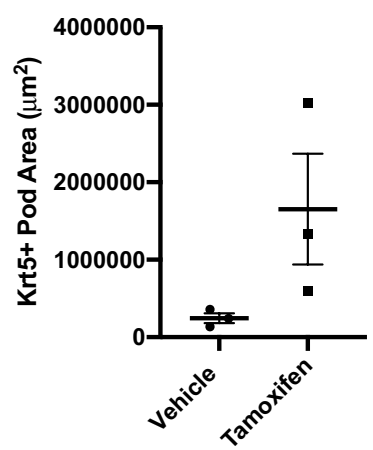

Extended Data Fig. 9 | Loss of TrkB in mesenchymal cells worsens outcomes following sterile and infectious lung injuries. a, H\&E staining and ATS lung injury scores of tamoxifen and corn oil exposed Pdgfr $\alpha^{\text {CreERT2: TrkB LoxPLLxP }}$ mice 24 hours after acid-induced lung injury ( $n=4$ mice per group). b-d, H\&E

(b), PDPN (c) and Krt5 (d) staining of tamoxifen and corn oil exposed Pdgfra ${ }^{\text {CreeRT2:Trk }}$ LoxP/LoxP mice 21 days after infection with PR8 influenza ( $n=3$ mice per group). Statistical analysis was performed with a two-tailed Student's t-test or ANOVA, where appropriate. For panels (a) and (d), data is shown as the mean + /- SEM. Statistical significance was determined with a two-tailed Student's t-test (a) and (d). Scale bars, 100 $\mu \mathrm{m}$. 


\section{Sftpc CreERT2:Stat3LoxP/LoxP + Tamoxifen}

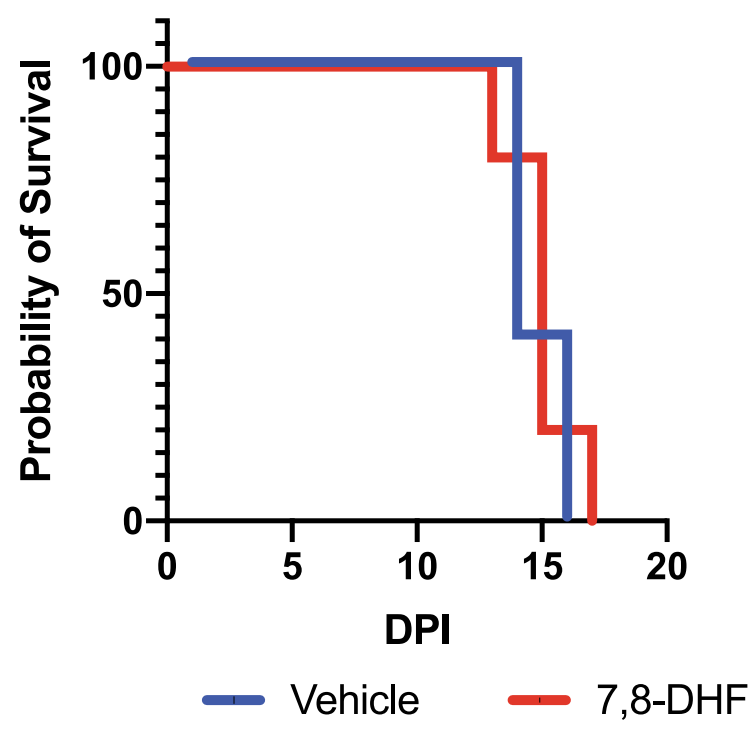

Extended Data Fig. 10 | The TrkB agonist 7,8-DHF is unable to rescue mice with an AT2-specific Stat3 deletion. Survival curves for Tamoxifen-exposed

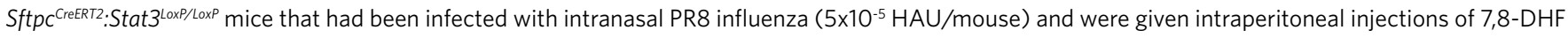
or vehicle every other day ( $n=5$ mice per group). The data analyzed using the Log-rank (Mantel-Cox) test and is not statistically significant. 


\section{Reporting Summary}

Nature Research wishes to improve the reproducibility of the work that we publish. This form provides structure for consistency and transparency in reporting. For further information on Nature Research policies, see Authors \& Referees and the Editorial Policy Checklist.

\section{Statistics}

For all statistical analyses, confirm that the following items are present in the figure legend, table legend, main text, or Methods section.

n/a $\mid$ Confirmed

$\square$ \.he exact sample size $(n)$ for each experimental group/condition, given as a discrete number and unit of measurement

$\square$ \ A statement on whether measurements were taken from distinct samples or whether the same sample was measured repeatedly

The statistical test(s) used AND whether they are one- or two-sided

$\square$ Only common tests should be described solely by name; describe more complex techniques in the Methods section.

Х $\square$ A description of all covariates tested

Х $\square$ A description of any assumptions or corrections, such as tests of normality and adjustment for multiple comparisons

$\square$ A full description of the statistical parameters including central tendency (e.g. means) or other basic estimates (e.g. regression coefficient)

AND variation (e.g. standard deviation) or associated estimates of uncertainty (e.g. confidence intervals)

$\square$ For null hypothesis testing, the test statistic (e.g. $F, t, r$ ) with confidence intervals, effect sizes, degrees of freedom and $P$ value noted

$\square$ Give P values as exact values whenever suitable.

Х $\square$ For Bayesian analysis, information on the choice of priors and Markov chain Monte Carlo settings

Х $\square$ For hierarchical and complex designs, identification of the appropriate level for tests and full reporting of outcomes

\ $\square$ Estimates of effect sizes (e.g. Cohen's $d$, Pearson's $r$ ), indicating how they were calculated

Our web collection on statistics for biologists contains articles on many of the points above.

\section{Software and code}

Policy information about availability of computer code

Data collection Cell sorting data was collected using MoFlo Astrios software (Summit (v 6.3.0.16900)) from Beckman Coulter. Confocal Images were acquired with the aid of Leica software (LAS X, v 3.0.13). Brightfield images of H \& E stained sections were obtained using Aperio software (ImageScope v. 12.4)

Data analysis

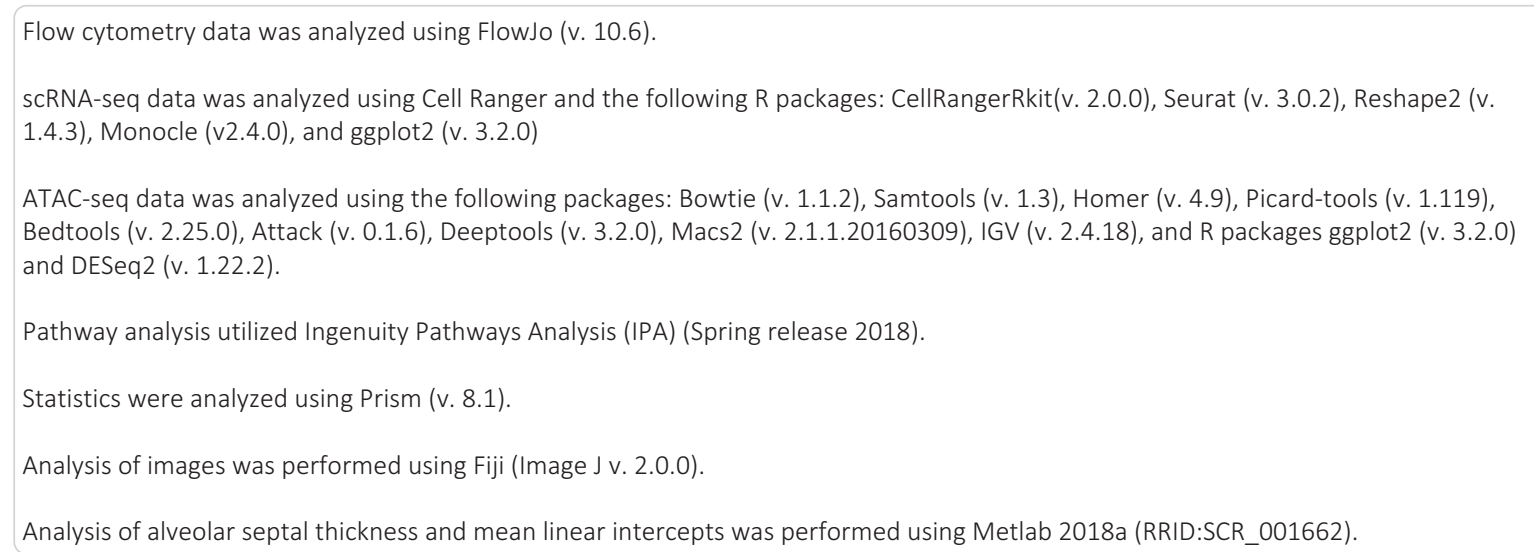


Policy information about availability of data

All manuscripts must include a data availability statement. This statement should provide the following information, where applicable:

- Accession codes, unique identifiers, or web links for publicly available datasets

- A list of figures that have associated raw data

- A description of any restrictions on data availability

Sequencing (ATAC-seq and scRNA-seq) data generated during this study has been deposited in the GEO database with the primary accession number GSE132535 (https://www.ncbi.nlm.nih.gov/geo/query/acc.cgi?acc=GSE132535).

\section{Field-specific reporting}

Please select the one below that is the best fit for your research. If you are not sure, read the appropriate sections before making your selection. $\bigotimes$ Life sciences $\quad \square$ Behavioural \& social sciences $\quad \square$ Ecological, evolutionary \& environmental sciences

For a reference copy of the document with all sections, see nature.com/documents/nr-reporting-summary-flat.pdf

\section{Life sciences study design}

All studies must disclose on these points even when the disclosure is negative.

Sample size Sample sizes were not predetermined and were based on previous experience (footnotes 19 and 39) that resulted in generation of statistically-significant data.

Data exclusions No data were excluded.

Replication All data was reliably reproduced. The number of repeats is indicated in each figure legend.

Randomization Mice were randomly assigned to groups consistent with genotype. Human samples were selected using predefined criteria which was having a pathology report that identified diffuse alveolar damage or normal lung parenchyma (control). Control lungs were age-matched to damaged lung.

Blinding Histologic sections were coded and analyzed by a blinded observer. Once all images had been graded, blinding was reversed for analysis by another investigator. Similarly, we used coding to conceal the conditions from which BAL samples used for ELISAs and cDNA used for RT-PCR were obtained. The samples were subsequently decoded by the primary investigator only after the data was acquired and analyzed. The scientist performing ELISAs and RT-PCR was unaware of the conditions from which the samples were obtained. Furthermore, the scientist collecting the BAL and RNA specimens was also unaware of the treatment conditions.

Determination of mortality in the influenza experiments did not involve blinding as it is necessary for us to accurately label our mouse cages and post relevant information on the experimental conditions on cage cards to allow for the mice to properly be cared for by our staff.

\section{Reporting for specific materials, systems and methods}

We require information from authors about some types of materials, experimental systems and methods used in many studies. Here, indicate whether each material, system or method listed is relevant to your study. If you are not sure if a list item applies to your research, read the appropriate section before selecting a response.

Materials \& experimental systems

\begin{tabular}{l|l}
\hline $\mathrm{n} / \mathrm{a}$ & Involved in the study \\
\hline & $\bigotimes$ Antibodies \\
$\square$ & $\bigotimes$ Eukaryotic cell lines \\
$\square$ & $\square$ Aalaeontology \\
$\square$ & $\square$ Animals and other organisms \\
$\square$ Clinical data
\end{tabular}

Methods

n/a Involved in the study

\ $\square$ ChIP-seq

$\square \bigotimes$ Flow cytometry

\ $\square$ MRI-based neuroimaging

\section{Antibodies}

Antibodies used

Please see Supplementary Table 2

Validation

Other than the HT2-280 antibody from Dr. Dobbs, all of the other antibodies are commercially available and validated by the manufacturer for use in mice (flow cytometry) or humans (MACs sorting). Validation of HT2-280 for these purposes has been 


\section{Eukaryotic cell lines}

Policy information about cell lines

Cell line source(s)

Primary AT2 cells from murine and human lungs were sorted and cultured with either primary lung fibroblasts (PDGFRA+) in the case of murine organoids, or MRC5 fibroblast cell line in the case of human organoids. The Human MRC5 fibroblast line was obtained from ATCC

Authentication

AT2 cells were defined as follows:

Mouse: Epcam+;CD45-;CD31-;PDGFRA-;CD24-; Sca-1-

Human: Positive for HT2-280 antibody

Fibroblasts:

Mouse PDGFRA+

Human MRC5 fibroblast line was obtained from ATCC and were not independently authenticated by our lab.

Mycoplasma contamination

Mycoplasma testing was performed on MRC5 fibroblast cell line and was negative. Mycoplasma testing was not performed on primary cells.

Commonly misidentified lines (See ICLAC register)

No commonly misidentified cell lines were used in this study.

\section{Animals and other organisms}

Policy information about studies involving animals; ARRIVE guidelines recommended for reporting animal research

Laboratory animals

Mus musculus of both sexes were used. Experimental mice were 8-12 weeks of age. Donor mice for isolation of AT2 and lung fibroblasts were 4-6 weeks of age. SftpcCreERT2 mice were generously donated by Harold A. Chapman at the University of California San Francisco. TrkbEGFP mice were generously donated by David D. Ginty at Harvard University. TrkBLoxP mice were generated by Louis F. Parada, currently at Memorial Sloan Kettering Cancer Center, and were shared with us by James $\mathrm{O}$. McNamera at Duke University. All other mice were obtained from the Jaxon Laboratory - C57BL/6 (stock \#000664), BdnfCre (stock \#030189), BdnfLoxP (stock \#004339), Hopx3FlagGFP (stock \#029271), PdgfraCreERT2 (stock \#018280), R26RTdTomato (stock \#007914), and Stat3LoxP/LoxP (stock \#016923).

Wild animals

No wild animals were used in this study.

Field-collected samples

This study did not involved samples collected from the field.

Ethics oversight

The protocol was approved by the IACUC at the Children's Hospital of Philadelphia and University of Pennsylvania.

Note that full information on the approval of the study protocol must also be provided in the manuscript.

\section{Human research participants}

Policy information about studies involving human research participants

Population characteristics Donated and de-identified post-mortem lungs rejected for transplant were used.

Recruitment Samples of uninjured, de-identified human lungs were obtained from non-utilized lungs donated for organ transplantation via an established protocol for the Prospective Registry of Outcomes in Patients Electing Lung Transplant study approved by University of Pennsylvania Institutional Review Board with informed consent in accordance with institutional procedures.

Ethics oversight

University of Pennsylvania Institutional Review Board

Note that full information on the approval of the study protocol must also be provided in the manuscript.

\section{Flow Cytometry}

\section{Plots}

Confirm that:

$\triangle$ The axis labels state the marker and fluorochrome used (e.g. CD4-FITC).

$\bigotimes$ The axis scales are clearly visible. Include numbers along axes only for bottom left plot of group (a 'group' is an analysis of identical markers).

$\bigotimes$ All plots are contour plots with outliers or pseudocolor plots.

$\bigotimes$ A numerical value for number of cells or percentage (with statistics) is provided. 


\section{Methodology}

Sample preparation

Murine lung tissue was digested into a single-cell suspension using dispase (Corning catalog \#354235), collagenase (Roche catalog \#10103578001), and DNase (Roche catalog \#10104159001).

Instrument

MoFlo Astrios

Software

Summit (v 6.3.0.16900) by Beckman-Coulter was used for sorting.

Cell population abundance

Information on cell abundance is included in Extended Figure 1 and Extended Figure 8. Data on cell purity is in Figure 1B.

Gating strategy

For all flow experiments the initial sorting material was a single-cell suspension of murine lung tissue. The FSC/SSC gates were set to exclude debris. Boundaries between positive and negative cells were set using unstained samples. Extended Figure 1 and Extended Figure 8 show our gating strategies in detail.

$\bigotimes$ Tick this box to confirm that a figure exemplifying the gating strategy is provided in the Supplementary Information. 